\title{
Smooth muscle cell-specific deletion of Col15a1 unexpectedly leads to impaired development of advanced atherosclerotic lesions
}

\author{
Brittany Grace Durgin \\ Old Bridge, New Jersey \\ Master of Science in Biological and Physical Sciences \\ University of Virginia, 2014 \\ Bachelor of Arts in Cell Biology and Neuroscience \\ Rutgers University-Douglass College, 2010
}

A Dissertation Presented to the Graduate Faculty of the University of Virginia in Candidacy for the Degree of Doctor of Philosophy

Department of Molecular Physiology and Biological Physics

University of Virginia

May 2017

\author{
Dr. Jessica J. Connelly \\ Dr. Gary K. Owens \\ Dr. Coleen A. McNamara \\ Dr. Brant E. Isakson \\ Dr. Norbert Leitinger
}


(c) Copyright by

Brittany Grace Durgin

All Rights Reserved May 2017 


\section{Abstract}

Atherosclerotic plaque rupture or erosion with subsequent embolic events is a major cause of sudden death from myocardial infarction or stroke ${ }^{1-6}$. Plaque stability is classically characterized by a fibrous cap rich in $\mathrm{ACTA} 2^{+}$presumed smooth muscle cells $(\mathrm{SMC})$ and collagen with a paucity of $\mathrm{CD} 68^{+}$presumed macrophages ${ }^{7-10}$. The dogma in the field is that SMC produce collagens that in turn provide tensile strength and stability to atherosclerotic plaques. This has largely been supported by evidence that SMC can produce collagens in vitro ${ }^{11-13}$. However, there is no direct evidence in vivo that SMC produce collagens as: 1) collagens are secreted molecules making it challenging to identify their cell source in vivo; and 2) recent lineage tracing studies by our lab ${ }^{14-16}$ and others ${ }^{17-}$ ${ }^{20}$ have shown that marker genes used to identify cell type within lesions are nonspecific. For example, SMC marker genes (e.g. ACTA2) can be expressed by myeloid-derived cells and endothelial cells and SMC can express marker genes of macrophages (e.g. CD68 and LGALS3) ${ }^{14-20}$. Therefore major unanswered questions remained in the field as to whether SMC are the primary source of collagens in vivo and if SMC produced collagens in turn impact plaque development.

We are interested in type XV collagen alpha 1 (COL15A1) as we identified a single nucleotide polymorphism (SNP) within human COL15A1 associated with age-related atherosclerosis disease risk ${ }^{21}$. COL15A1 is a non-fibrillar collagen upregulated in human and mouse atherosclerosis that has been shown to link large collagen fibers to confer extracellular matrix (ECM) and tissue stability ${ }^{21-26}$. 
Moreover, siRNA mediated knockdown of Col15a1 in cultured human aortic SMC results in an increase in SMC migration and a decrease in SMC proliferation ${ }^{21}$. Taken together, we hypothesized that SMC produced COL15A1 would be critical in late stage atherosclerotic lesion stability through promoting organization of the extracellular and collagen matrix in the lesion and fibrous cap.

We demonstrate that SMC specific lineage tracing $\left(\mathrm{YFP}^{+}\right), \mathrm{SMC}$ specific Col15a1 knockout mice have impaired lesion development as compared to wild type controls and fail to form advanced lesions despite 18 weeks of Western diet feeding. SMC Col15a1 knockout lesions exhibit a drastic reduction in lesion size, overall cell number, and complexity as exhibited by an increased proportion of macrophages $\left(\mathrm{YFP}^{-} \mathrm{LGALS3}^{+} / \mathrm{DAPI}^{+}\right)$and decreased proportion of SMC per total cells $\left(\mathrm{YFP}^{+} / \mathrm{DAPI}^{+}\right)$and proliferating $\mathrm{SMC}\left(\mathrm{YFP}^{+} \mathrm{Ki}^{+} 7^{+} / \mathrm{DAPI}^{+}\right)$as compared to wild-type control lesions. Loss of SMC produced COL15A1 also led to a reduction in lesion and medial collagen content and organization which likely contributed to an increase in carotid artery passive tone. In vivo RNA-seq analysis on SMC Col15a1 knockout and wild type control lesions suggest one mechanism of action to explain these effects is likely through SMC-COL15A1 mediated inhibition of pro-atherogenic inflammatory pathways (ex. lipopolysaccharides, TNF, TGF $\beta$, etc.) involved in lesion development. Combined, these results provide the first direct evidence that a SMC-derived collagen, COL15A1, is critical in lesion development. 


\section{Dedication}

I dedicate this to my grandfather, Arthur Labuz. You were the heart of my family and a testament to hard work, perseverance, and inner strength. I hope I have inherited an ounce of these qualities from you and have made you proud. 


\section{Acknowledgements}

I am incredibly grateful to have had the opportunity pursue a PhD degree at UVA. It has been quite the journey and I have worked with some fantastic people in my time here.

I would like to begin with a huge thank you to my mentors Jessica Connelly and Gary Owens. Jess, you have been a true champion of mine from the start. Thank you for encouraging me, teaching me how to be a rigorous scientist, pushing me to be better, and reminding me to take the time to think! Gary, I have grown exponentially as a scientist since joining your lab and fielding your questions. Thank you for taking me on, believing in me, and pushing me to be great.

I would like to thank my committee members Brant Isakson, Coleen McNamara, and Norbert Leitinger for supporting both me and my science. You have all always strived to help me succeed, for which I am eternally grateful.

I also have had the pleasure of having the best co-workers. Thank you Travis Lillard and Themis Karaoli; you have always been helpful, fun, and patient with me. To the Owens Lab, you are quite an amazing and talented crew. I would like to say a special thank you to Missy Bevard and Mary McCanna for their help in histology and to Rupa Tripathi for assisting me in all my cell culture experiments; Rupa thank you also for always making me smile. Olga Cherepanova, thank you for answering my numerous questions and for your constant guidance and support both scientifically and emotionally. Delphine 
Gomez and Alexandra Newman, your friendship has been instrumental to my sanity, happiness, and science.

I would also like Emily Mercadante for being a true friend and support for me through my PhD. Thank you Katie Marguileux, Caitlin Naylor, Glen Hirsh, and Jessica Harakal for your friendship and for hosting social events to force me out of the lab. Anahita Madon, Karen Hidalgo and Veronica Pakit, thank you for the laughs and giving me perspective. To my partner Chris Smolko, you are absolutely wonderful.

Last but not least, thank you to my biggest support system, my family. To my sisters Caitlin and Keriann Durgin, you are everything; thank you for being my rocks. Finally, to my parents Lauren and John Durgin, thank you for your unwavering support and faith in my abilities. I made it. 


\section{Table of Contents}

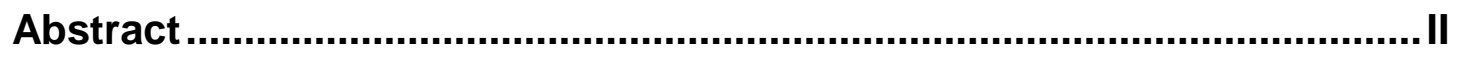

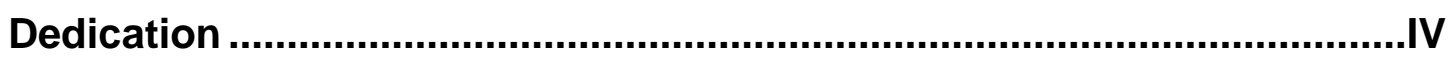

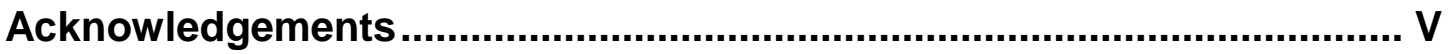

Table of Contents .......................................................................................

List of Abbreviations .....................................................................................

List of Synonymous Terms and Phrases .............................................. XIV

List of Figures and Tables ................................................................... XV

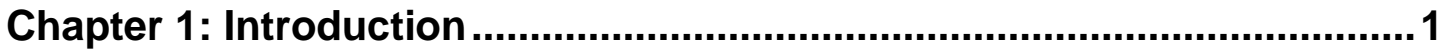

1. The Public Health Burden of Atherosclerosis ............................... 2

1.1 Atherosclerosis is the major underlying cause of cardiovascular

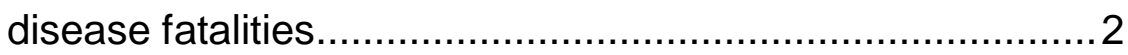

2. The Importance of Collagens and Smooth Muscle Cells (SMC) to Atherosclerotic Plaque Development and Stability .....

2.1 An intact $\mathrm{ACTA}^{+}$and collagen-rich fibrous cap and lesion are hallmarks of stable human atherosclerotic plaques.............. 4

2.2 ACTA2 ${ }^{+}$SMC are important for fibrous cap stability.............

2.3 Recent rigorous lineage tracing studies provide definitive evidence that cell marker gene expression is not specific within atherosclerotic lesions

2.4 SMC lineage tracing studies show SMC undergo oligoclonal expansion within lesions 
2.5 SMC conditional knockout of pluripotency genes Klf4 and Oct4 show SMC phenotypically switch in vivo and these phenotypic transitions can have both an atheroprotective and atheropromoting role in lesion development

2.6 Collagens are important for maintenance of fibrous cap and atherosclerotic plaque stability and resilience to rupture $\ldots . .11$

2.7 Collagen mediated signaling 12

2.8 Global collagen knockout studies have provided evidence that collagens can influence atherosclerotic plaque development

2.9 Cultured SMC can produce collagens and collagen state can influence SMC function in vitro

3. An Overview and Rationale for the Study of Collagen Type XV, Alpha 1 (COL15A1) 16

3.1 The rationale for studying collagen type $\mathrm{XV}$, alpha 1 (COL15A1) 16

3.2 COL15A1 is unique in that it is a non-fibrillar proteoglycan-like collagen 17

3.3 COL15A1 functions as a fibrillar collagen organizer important for tissue integrity and influences cell responses to injury and disease 19

3.4 COL15A1 is present and upregulated in atherosclerotic disease with Col15a1 expression influencing SMC function 
Chapter 2: Smooth muscle cell-specific deletion of Col15a1 unexpectedly leads to impaired development of advanced atherosclerotic lesions ....24

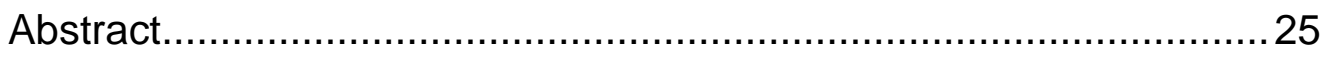

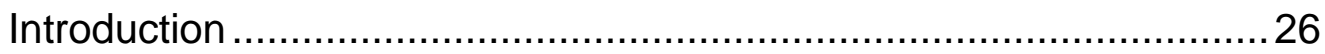

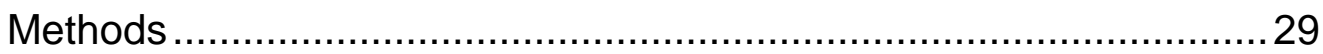

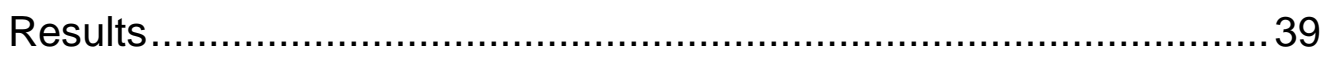

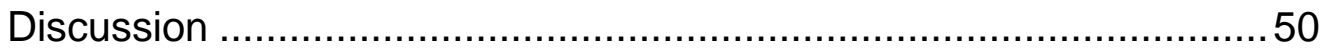

Chapter 3: Conclusions and Future Directions …..................................77

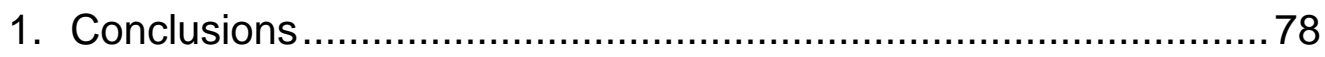

2. Discussion and Future Directions ............................................. 78

2.1 SMC produced COL15A1 is important in the formation and organization of fibrillar collagens within the vessel wall and in atherosclerotic lesions

2.2 SMC produced COL15A1 impacts various pro-inflammatory pathways and nearly all immune cell type

2.3 COL15A1 as a modulator of cell proliferation 85

2.4 Is there an alternative cell source of COL15A1 within lesions?

2.5 Does loss of SMC COL15A1 production result in delayed and/or halted progression of atherosclerotic lesion development? Does COL15A1 affect all locations of the arterial tree 
similarly? 90

2.6 In our previous human studies, we found evidence that the human COL15A1 SNP is associated with decreased COL15A1 expression and increased age-related risk for atherosclerosis. In contrast, our mouse studies show loss of SMC produced COL15A1 results in attenuated lesion development. How can we rectify these observations? Moreover, does modifying COL15A1 content within lesions have therapeutic potential? 92

2.7 What does COL15A1 directly interact with? Does it bind specific cell surface receptors? 95

2.8 The N-terminal domain and part of the collagenous domain of COL15A1 have glycosaminoglycan (GAG) binding sites containing mostly chondroitin sulfates. Could these GAG chains on $\mathrm{COL} 15 \mathrm{~A} 1$ be playing a role in the initiation of atherosclerotic lesion development? 98

2.9 What about COL15A1 C-terminal cleavage product restin? Could it be playing a role in atherosclerotic lesions? 100

2.10 Does COL15A1 have a role in the microvasculature? 102

2.11 Is there a connection between IL1 $\beta$ and COL15A1? 103

2.12 Is COL15A1 likely to be involved in human plaque 
erosion events? ....................................................... 105

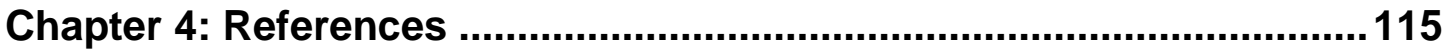




\section{List of Abbreviations}

ACTA2 - Smooth muscle alpha actin, SMaA

ApoB48 - Apolipoprotein B 48

ApoB100 - Apolipoprotein B 100

Apoe - Apolipoprotein E

BCA - Brachiocephalic artery

BMDM - Bone marrow derived macrophages

BxPC-3 - Pancreatic epithelial adenocarcinoma

CABG - Coronary Artery Bypass Graft

CASP3 - Cleaved Caspase 3

CD31 - Platelet endothelial cell adhesion molecule

CD68 - Macrophage antigen CD68

COL15A1 - Collagen type XV alpha 1 protein

COL15A1 - Human gene

Col15a1 - Mouse gene

Cre-ER ${ }^{\mathbf{T} 2}$ - Tamoxifen inducible estrogen receptor bound Cre-recombinase

CVD - Cardiovascular disease

DDR1 - Discoidin domain receptor 1

DNA - Deoxyribonucleic acid

D98 AP2 cells - Human cervical carcinoma cell line

E-cadherin - Endothelial cadherin

EOMA - Endothelial cell mouse hemangioma

ER - Endoplasmic reticulum 
FISH - Fluorescence in situ hybridization

GAG - Glycosaminoglycan

H3K9me2 - Histone 3 lysine 9 dimethylation

HDL - High-density lipoprotein

HUVEC - Human umbilical vein endothelial cell

IFNY - Interferon gamma

IL1 $\beta$ - Interleukin 1 beta

IL1R1 - Interleukin 1 receptor 1

ISH-PLA - in situ hybridization-proximity ligation assay

IVUS - Intravascular ultrasound

KO - Knockout

LDL - Low-density lipoprotein

LGALS3 - Galectin 3, MAC2 antigen

MAC2 - Macrophage marker

MAC3 - Macrophage marker

MCP1 - Monocyte chemoattractant protein

MKi67 - Marker of Proliferation Ki67

MYH11 - Smooth muscle myosin heavy chain

NSTEMI - Non S-T elevated myocardial infarction

OCT - Optical coherence microscopy

PBS - Phosphate buffered saline

PBMCs - Peripheral blood monocytes

PDGFBB - Platelet derived growth factor beta 
PDGFBR - Platelet derived growth factor beta receptor

RNA - Ribonucleic acid

RNA Pol II - RNA polymerase II

ROBO- Roundabout family of proteins

SEM - Standard error of the mean

SLIT - Secreted extracellular matrix proteins

SM22 $\alpha$ - Smooth muscle protein 22 alpha

SMC - Smooth muscle cell

SNP - Single Nucleotide Polymorphism

STEMI - S-T Elevation Myocardial Infarction

TAGLN - Transgelin, smooth muscle 22 alpha

TEM - Transmission electron microscopy

TGF $\beta$ - Transforming growth factor beta

THP1 macrophages - Human monocytic cell line derived from an acute monocytic leukemia patient

TNF $\boldsymbol{\alpha}$ - tumor necrosis factor alpha

\section{List of Common Synonymous Terms and Phrases}

1) "Atherosclerotic plaque" is commonly used interchangeably with "atherosclerotic lesion"

2) "Collagen maturation" is synonymous with "collagen organization"

3) The "vessel wall" is anatomically referred to as "the media" 


\section{List of Figures and Tables}

\section{Chapter 2:}

Figure 1: Generating Col15a $1^{f / f t}$ mice and SMC specific Col15a $1^{f / f t}, \mathrm{SMC}$ lineage tracing mice. 56

Figure 2: Validation of specificity of SMC Col15a1 knockout, SMC lineage tracing mice 58

Figure 3: Loss of SMC-derived COL15A1 resulted in a marked decrease in lesion size 60

Figure 4: Genetic inactivation of Col15a1 in SMC reduced collagen fiber content and maturation, and vessel elasticity 62

Figure 5: Loss of SMC specific COL15A1 resulted in overall reductions in the number of $\mathrm{YFP}^{+} \mathrm{SMC}$-derived cells within the lesions but an increase in YFP ${ }^{-} \mathrm{LGALS}^{+}$macrophage cells 64

Figure 6: SMC-derived cell populations were reduced with SMC Col15a1 knockout 66

Figure 7: SMC specific Col15a1 knockout was associated with a decrease in lesion SMC proliferation 68

Figure 8: SMC Col15a1 knockout resulted in a decrease in lesion cell death 70

Figure 9: SMC specific Col15a1 knockout resulted in downregulation of immune cell processes that impact atherosclerotic

plaque formation 
Figure 10: COL15A1 is pervasive within advanced human atherosclerotic plaques and localized to the fibrous cap and

intraplaque microvessels

Table 1: No differences in cholesterol, triglycerides, or body weight

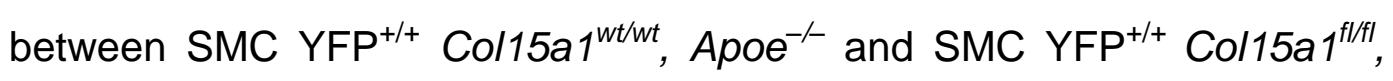
Apoe $^{-/-}$mice .................................................................... 76

Table 2: Genotyping and excision primers for Col15a1 .....................76

Chapter 3: 77

Figure 11: Preliminary data on SMC Col15a1 knockout (KO) cells generating a collagen matrix. 107

Figure 12: In situ hybridization-proximity ligation assay (ISH-PLA) of serine 5 phosphorylated RNA polymerase II (RNA Pol II) bound to Col15a1 promoter 109

Figure 13: Preliminary data from SMC IL1R1 knockout atheroprevention and anti-IL1 $\beta$ atherointervention studies 111

Figure 14: In vivo RNA-seq data list of genes upregulated and downregulated with SMC Col15a1 knockout 
Chapter 1:

Introduction 


\section{The Public Health Burden of Atherosclerosis.}

\subsection{Atherosclerosis is the major underlying cause of cardiovascular disease fatalities.}

Cardiovascular disease (CVD) is the leading cause of death in modern society. Specifically, CVD accounts for approximately 17.5 million deaths worldwide $^{3}$. In the United States, heart disease accounts for $25 \%$ of total deaths (approximately 610,000 people) per year ${ }^{27,28}$. Moreover, CVD represents a huge financial burden as the direct medical cost in the United States alone was \$396 billion a year in 2012 and is projected to increase to $\$ 918$ billion a year by $2030^{29}$

To better combat CVD burden, it is important to understand the major underlying cause of CVD: atherosclerosis. Atherosclerosis is the accumulation of fat and cholesterol in the artery wall which forms a plaque that can restrict blood flow through the artery ${ }^{30}$. Rupture and erosion of these atherosclerotic plaques can cause acute embolic events which account for the majority of sudden deaths from myocardial infarction and stroke ${ }^{1,9,31-33}$. Atherosclerosis is a progressive disease with lesions forming, in some cases, as early as in utero and progressing with age ${ }^{30,34,35}$. Risk factors for atherosclerosis include age, genetic predisposition, smoking, diet, hypertension, elevated LDL cholesterol, diabetes, and obesity ${ }^{34,36-38}$. Of great concern, almost half of all Americans currently have either high cholesterol, high blood pressure, or smoke ${ }^{36,39}$. 
Despite the prevalence of atherosclerosis, we still do not understand how to prevent atherosclerosis-derived embolic events. The current standard of care for patients focuses on indirect treatment of the disease through ameliorating risk factors. This involves treatment with statins to lower circulating lipids, antihypertensive drugs to lower blood pressure, a daily low-dose aspirin to lower systemic inflammation, and certain lifestyle interventions such as improving diet, increasing exercise, and quitting smoking ${ }^{40}$. Patients who develop late stage, highly occlusive atherosclerosis may also undergo surgical interventions including implantation of drug-eluting stents, coronary artery bypass graft (CABG), or carotid endarterectomy to restore blood flow ${ }^{40}$. A major issue with these intervention approaches to the treatment of atherosclerosis is that: 1) pharmaceutical and lifestyle interventions attempt to prevent further lesions from forming and do not focus on treating existing plaques; and 2) surgical interventions are typically stop gap measures to ameliorate a critically occluded vessel $^{40}$. Currently, there are no therapeutic strategies available that can target late stage atherosclerotic plaques or focus specifically on enhancing plaque stability and/or on plaque resolution as a means of preventing heart attack and stroke. 
2. The Importance of Collagens and Smooth Muscle Cells (SMC) to Atherosclerotic Plaque Development and Stability.

\subsection{An intact $\mathrm{ACTA}^{+}$and collagen-rich fibrous cap and lesion are hallmarks of stable human atherosclerotic plaques.}

In general, atherosclerotic lesion development occurs as a consequence of lipid retention in the vessel wall. This leads to the recruitment of macrophages, which uptake lipids, and form foam cells. In response to these changes, the contractile SMC undergo a phenotypic change after which they migrate into and proliferate within the lesion. As recently shown by Shankman et al., SMC can become foam-cell like and contribute to plaque destabilization ${ }^{15}$. In addition, SMC form a part of the fibrous cap wherein they are believed to secrete collagens and extracellular matrix components which stabilize the lesions. Importantly, a stable plaque is associated with decreased incidence of plaque rupture $e^{8,41-43}$.

Seminal work by Davis et al. in 1993 identified that intact human lesions were characterized as having small lipid pools and an increased ratio of $\mathrm{ACTA}^{+}$ presumed smooth muscle cells $(\mathrm{SMC})$ relative to $\mathrm{CD} 68^{+}$presumed macrophages in the fibrous cap; ulcerated, thrombotic plaques had the exact opposite characterization ${ }^{7}$. Similarly, recent work by renowned cardiovascular pathologist Renu Virmani has shown that most plaques correlated with fatal coronary thrombosis events undergo rupture or erosion ${ }^{1,9,31,43,44}$. Plaque ruptures, which cause the majority (55-70\%) of fatal coronary events, are characterized by fibrous caps that are thin, fractured, and $\mathrm{ACTA}^{+}$and collagen-poor that also 
contain large-lipid rich necrotic cores exposed to the lumen which promotes thrombus formation ${ }^{1,2,9,31,32,45,46}$. Notably, mouse models of atherosclerosis possess these same characteristics though with an intact fibrous cap as mice lesions are not thought to undergo spontaneous plaque rupture $e^{1,9,43,45,47,48}$. Therefore, we use mouse models of atherosclerosis to assess surrogate indices of plaque stability including lipid-deposition, necrotic core size, fibrous cap size, collagen content, intraplaque hemorrhage, and the ratio of SMC to macrophages. Plaque erosion in humans, which account for between $30-40 \%$ of coronary fatalities, are characterized as being intact plaques abundant in $\mathrm{ACTA}^{+}$cells, proteoglycans, and non-fibrillar collagens that develop thrombi on their luminal surface ${ }^{1,32,49,50}$. Interestingly, recent optical coherence topography studies have shown that plaque rupture was associated with S-T segment elevation myocardial infarction (STEMI) while plaque erosion was more closely associated with non-STEMI events ${ }^{50,51}$ suggesting a connection between plaque features and heart attack event type. Taken together, plaque rupture and erosion lesions with an increase $\mathrm{ACTA}^{+}$cells, presumed SMC, and extracellular matrix content are associated with plaque stability.

\section{2. $\mathrm{ACTA}^{+}$presumed $\mathrm{SMC}$ are important for fibrous cap stability.}

Vascular SMC within the vessel wall are identified by their expression of SMC specific contractile genes smooth muscle alpha actin (ACTA/SMaA), smooth muscle 22 alpha (TAGLN/SM22 $\alpha$ ), smooth muscle myosin heavy chain (MYH11), calponin, and smoothelin ${ }^{42,52,53}$. Logically, the cells within the fibrous 
cap that express $\mathrm{ACTA}^{+}$have long been assumed to be of SMC origin. The dogma in the field has been that SMC migrate into the lesions, proliferate in response to environmental cues, and localize to the fibrous cap where they secrete extracellular matrix to enhance plaque stability ${ }^{8}$. This has been supported by co-localization of $\mathrm{ACTA}^{+}$and collagen in the fibrous cap and by extensive studies showing treatment of cultured SMC with factors present within lesions (e.g. transforming growth factor beta (TGF $\beta$ ), platelet derived growth factor beta (PDGFBB), and oxidized phospholipids) can induce SMC production of collagen ${ }^{11,12,54-56}$. However, definitive in vivo evidence supporting this dogma is lacking as: 1) collagens are secreted molecules meaning that co-localization of collagen to a cell does not prove that cell produced that collagen; and 2) as described in detail in the following section 2.3, numerous recent studies from our $\operatorname{lab}^{14-16}$ and several others ${ }^{17,20,57,58}$ using rigorous lineage tracing approaches have provided compelling evidence that one cannot distinguish cell origin within lesions in vivo based solely on cell marker gene expression.

\subsection{Recent rigorous lineage tracing studies provide definitive evidence that cell marker gene expression is not specific within atherosclerotic lesions.}

Notably, cells other than SMC, such as macrophages and endothelial cells, were found to express cell marker genes characteristic of SMC within lesions and vice versa, indicating cell marker genes are not specific and cannot be used to definitively determine cell origin in atherosclerotic plaques $^{14,15,17,19,20,58}$. We have shown using our tamoxifen inducible SMC specific 
lineage tracing mice on an $A p o e^{-/}$background that $>80 \%$ of SMC within advanced lesions lack expression of $A C T A 2^{15}$ as well as that $>30 \%$ of these SMC have activated multiple markers of macrophages including F4/80, CD11b and LGALS3 ${ }^{15}$; these observations were recently bolstered by a near identical study published by Albarran-Juarez et $a l .{ }^{59}$ In agreement with these observations, studies by Feil et al. and Chappell et al. using two separate SMClineage tracing mouse model systems have also shown that SMC can express both macrophage (i.e. MAC3, MAC2) and SMC (i.e. ACTA2) marker genes ${ }^{20,60}$. Within human lesions, Allahverdian et al. observed that between $40-50 \%$ of $\mathrm{CD} 8^{+}$cells co-express ACTA2 ${ }^{+58}$. Consistent with this, our lab has also used a novel in situ hybridization-proximity ligation assay (ISH-PLA) that detects SMC lineage memory mark histone 3 lysine 9 dimethylation (H3K9me2) on the Myh11 promoter in formaldehyde fixed, paraffin embedded tissue sections to show that approximately $20 \%$ of the $\mathrm{CD}^{+} 8^{+}$cells within human lesions are actually SMCderived $^{15,16}$.

Furthermore, myeloid-derived cells and endothelial cells can express markers of SMC. Caplice et al. showed in patients who have undergone a crossgender bone marrow transplant that bone marrow derived cells within atherosclerotic lesions express SMC marker ACTA2 ${ }^{17}$. Likewise, mouse studies where bone marrow transplant was followed by short term eight week atherogenic diet found that between $40-50 \%$ of bone marrow derived cells express ACTA2 ${ }^{61}$, but not SMC marker MYH11 within lesions. ${ }^{62}$ Separately, studies by Chen et al. using an endothelial cell specific lineage tracing mouse 
model (Cdh5-CreER $\left.{ }^{\top 2}\right)$ showed that lineage tagged endothelial cells express ACTA2 in atherosclerotic lesions and that in advanced human atherosclerotic lesions, SMC marker SM22 $\alpha$ co-localizes with endothelial cell marker CD31 ${ }^{19}$. Combined, these studies implicate rigorous lineage tracing systems as essential for accurately identifying cell origin within atherosclerotic plaques.

In light of these lineage tracing studies, it must be acknowledged that published studies conducted in the absence of lineage tracing in both mouse and human lesions have likely largely misidentified the origin of cells within atherosclerotic lesions. Therefore, we are only just beginning to accurately identify and understand the effects of cell-specific contributions to atherosclerotic lesion development and indices of stability.

\subsection{SMC lineage tracing studies show SMC undergo oligoclonal expansion within the lesions.}

SMC lineage tracing studies have also advanced our knowledge of how SMC accumulate within atherosclerotic lesions. Early in vivo studies by Benditt et al. looked at lesions from female heterozygous patients that express both isoenzymes of X-linked glucose-6 phosphate dehydrogenase and observed that the majority of the plaques from the 4 patients sampled had only one isozyme

expressed within lesions while the media contained both isozyme types ${ }^{63}$. As a result, Benditt et al. concluded that lesion cells are monoclonal $\left.\right|^{63}$. However, later work by Thomas et al. looking at the fraction of labeled porcine medial cells at baseline that accumulate in lesions after 30-60 days of hypercholesterolemic 
diet, found that lesions were polyclonal ${ }^{63,64}$. In agreement with Thomas et al., separate SMC lineage tracing studies by Feil et al. and Chappell et al. have provided compelling evidence that SMC undergo oligoclonal expansion as a means of populating atherosclerotic lesions ${ }^{20,60}$. Intriguingly, Feil et al. and Chappell et al. also provide evidence that a single SMC clone can express both macrophage (i.e. MAC3, MAC2) and SMC (i.e. ACTA2) marker genes ${ }^{20,60}$. Together, these studies suggest that SMC accumulation in lesions occurs in part through SMC oligoclonal expansion. However, whether SMC accumulation involves oligoclonal expansion in conjunction with migration and/or clonal selection and what the order of operations is for how SMC move from the media to the lesion remain to be tested.

\subsection{SMC conditional knockout of pluripotency genes KIf4 and Oct4 show SMC phenotypically switch in vivo and these phenotypic transitions can have both an atheroprotective and atheropromoting role in lesion development.}

In our lab, we developed novel tamoxifen-inducible SMC specific lineage tracing mice: Myh11-CreER ${ }^{\text {T2 }}$ ROSA26 floxed STOP eYFP, Apoe ${ }^{-/ 14-16}$. This inducible SMC lineage tracing mouse model permanently labels SMC with enhanced yellow fluorescent protein (YFP) with >95\% efficiency in large conduit vessels after a series tamoxifen injections ${ }^{15}$. Our lab crossed these SMC lineage tracing mice with floxed mice of pluripotency genes KIf4 and Oct4 to explore how these genes affect SMC phenotype transitions in atherosclerotic lesions and 
contribute to atherosclerotic lesion development. Work by Shankman et al. found that SMC KIf4 knockout mice fed a Western diet for 18 weeks developed lesions that were smaller with increased indices of plaque stability ${ }^{15}$. The authors went on to show that SMC KLF4 is important for SMC plasticity as SMC Klf4 knockout results in a reduction of SMC-derived macrophages which are believed to exacerbate atherosclerosis ${ }^{15,65}$. Interestingly, Shankman et al. show by transmission electron microscopy (TEM) for the first time in vivo that SMC, like macrophages, can phagocytosis lipids in lesions and therefore functionally change ${ }^{15}$. In vitro studies by Vengrenyuk et al., however, suggest that the SMCderived macrophages do not phagocytose as well as traditional macrophages ${ }^{65}$.

In contrast, SMC lineage tracing, SMC specific Oct4 knockout mice fed 18 weeks Western diet exhibited larger lesions with reduced indices of plaque stability including reduced SMC accumulation in the lesion and fibrous $\operatorname{cap}^{14}$. Ex vivo aortic explants from SMC Oct4 knockout and wild type mice as well as numerous studies in cultured SMC provide evidence that the reduction in SMC accumulation within SMC Oct4 knockout lesions is likely due to impaired SMC migration $^{14}$. The lab concluded that Oct4 expression within SMC was atheroprotective as it is important for SMC migration into the lesion and localization at the fibrous cap. Conversely, KIf4 expression is atheropromoting as it promotes SMC phenotypic transitions to what we believe to be pro-atherogenic SMC-derived macrophages within lesions ${ }^{14}$. Combined, these studies provided the first evidence that SMC can be both advantageous and detrimental to atherosclerotic lesion development and indices of stability. 


\subsection{Collagens are important for maintenance of fibrous cap and atherosclerotic plaque stability and resilience to rupture.}

Collagens are prominent, pervasive, and essential components of vessel walls and atherosclerotic lesions. Collagens are defined by their distinctive triple helical protein repeat of Gly-X-Y where Gly stands for glycine and $X$ and $Y$ are frequently proline, lysine, or hydroxylate versions of the two ${ }^{66,67}$. In the normal human vasculature, the media or vessel wall consists of a plethora of collagen types, elastin, and proteoglycans ${ }^{21,68-70}$. Collagens reside and are prevalent in both the interstitial matrix and the basement membrane of the vasculature. The interstitial matrix resides in the extracellular space between cells and consists of mostly fibrillar collagens as well as a variety of proteoglycans, polysaccharides, and fibronectin ${ }^{66}$. The basement membrane is a specialized matrix in direct contact with both cells and the interstitial matrix that consists of a wide variety of extracellular components (e.g. collagen types IV, XV, XVIII, laminin, etc ${ }^{71,72}$ ). While the interstitial matrix functions mainly in structural support ${ }^{66}$, the basement membrane is thought to be important in tissue flexibility, organization, and cell anchorage; both regions can contribute to cell signaling ${ }^{67,72,73}$.

In advanced human lesions, collagens make up $60 \%$ of the total aortic protein with fibrillar collagen type I composing the majority ${ }^{74-76}$. In addition to fibrillar collagen type I, fibrillar collagen type III, basement membrane collagen types IV and XV, and other collagens are also increased, though to a lesser extent, in atherosclerotic lesions ${ }^{21,68-70}$. Of importance, these collagens localize to the fibrous cap and shoulder regions of atherosclerotic plaques, which are 
important for stability ${ }^{75,77,78}$. It has been well established that increased fibrillar collagen in the fibrous cap is correlated with resilience to mechanical stress and prevention of plaque rupture in human atherosclerotic plaques ${ }^{46,79-81}$. Consequently, fibrillar collagens have been considered structural scaffolds important for the enhancement and maintenance of plaque stability.

\subsection{Collagen mediated signaling.}

Collagens also influence cell signaling and function. One method of collagen mediated signaling is through direct binding to integrin and/or discoidin domain receptors present on a cell's surface ${ }^{13,82,83}$. Integrins are cell-surface receptors that mediate cell adhesion and binding to GFOGER, GLOGER, or GASGER fibrillar collagen protein motifs ${ }^{13,82,83}$. Discoidin domain receptors (DDR1, DDR2, and DDR3) are receptor tyrosine kinases that also bind collagens; DDR2 specifically binds to fibrillar collagen motif GVMGFO $^{13,82,83}$. Integrins and discoidin domain receptors are found on both SMC and macrophages. Certain types of integrins and discoiden domain receptors have also been shown to contribute to atherosclerotic lesion development and can act in a cell-specific manner ${ }^{13,82-84}$. For example, when global DDR $1^{-/}$and wild type animals were transplanted with DDR $1^{-/}$bone marrow, global DDR $1^{-/}$animals exhibited attenuated lesion development compared to wild type animals ${ }^{85-87}$. In contrast, global $\mathrm{DDR} 1^{-/-}$animals transplanted with $\mathrm{DDR} 1^{+/+}$bone marrow developed larger lesions than control animals suggesting a cell-specific function for the DDR1 receptor in atherosclerotic lesion development ${ }^{85-87}$. 
Separately, cleavage of collagens by matrix metalloproteases in the extracellular space can result in generation of bioactive signaling peptides called "matrikines" "2,88-90. These matrikines can have properties and signaling capabilities completely separate from that of their parent collagen ${ }^{72,88,89}$. For example, cleavage of collagen type IV generates six different matrikines, three of which (e.g. arresten, canstatin, and tumstatin) are anti-angiogenic ${ }^{71,88,91,92}$. The diversity and presence of matrikines in the extracellular milieu adds another layer of complexity to collagen action and signaling as well as collagen impact on cellular function. Collagens are therefore not just inert scaffolds for structural stability, but dynamic contributors to cell signaling and function.

\subsection{Global collagen knockout studies have provided evidence that collagens can influence atherosclerotic plaque development.}

Several studies in global collagen knockout animals have provided evidence that collagen production can also influence atherosclerotic lesion development and composition. Work by Fukumoto et al. in global transgenic collagenase resistant Col1a1 mice fed ten weeks of atherogenic diet found an increase in collagen content and decrease in number of and proliferating ACTA2 ${ }^{+}$ cells, but observed no difference in lesion size or $\mathrm{MAC3}^{+}$cell number ${ }^{93}$. A separate study using global knockout of non-fibrillar multiplexin collagen XVIII (Col18a1) in $\mathrm{Apoe}^{--}$mice fed a Western diet for sixteen weeks also observed a decrease in $\mathrm{ACTA}^{+}$cells, but found an increase in lesion size and $\mathrm{MAC}^{+}$cell coverage $^{94}$. Similarly, global knockout of non-fibrillar collagen type VIII 
(Col8a1/a2) in $\mathrm{Apoe}^{-/-}$mice resulted in a decrease in $\mathrm{ACTA}^{+}$cells and total collagen content with no difference in lesion size or MAC2 cells after six or twelve weeks of atherogenic diet ${ }^{95}$. Together these studies provide evidence that collagen production and organization influences atherosclerotic lesion development and can affect cell composition of lesions. However, major limitations exist with these studies including that: 1) the cellular source of collagen is not identified; 2) the studies do not determine whether cell source of collagen is crucial to the observed phenotype; and 3) there is a possibility that global loss of a specific collagen since birth may have caused biological changes to compensate for the loss of the collagen throughout mouse development. Therefore, while collagens are known to be important in atherosclerotic plaque progression and stability, there are major unanswered questions as to the identity of collagen producing cells in atherosclerotic lesions in vivo and whether cellspecific collagen production influence atherosclerotic lesion development.

\subsection{Cultured SMC can produce collagens and collagen state can influence SMC function in vitro.}

Studies of SMC and collagens in vitro suggest that SMC may be a collagen contributing cell source within atherosclerotic lesions. For example, cultured SMC treated with growth factors PDGFBB ${ }^{11,52,53,96-99}$ and TGF ${ }^{11}$ produce collagen type I, while oxidized phospholipid treatment increases SMC production of collagen type $\mathrm{VIII}{ }^{12,56}$; these factors are all found within atherosclerotic lesions. In contrast, cultured SMC treated with interferon gamma 
(IFNY) shows inhibition of SMC production of collagen type $\mathrm{I}^{11}$ indicating that SMC production of collagens is mediated in part by interactions with specific factors in its environment. In addition to SMC producing collagens, inhibiting SMC collagen production can also can influence SMC function. An example of this is seen in work by Adiguzel et al. where Col8a1/a2 ${ }^{-/}$knockout SMC exhibit reduced migration and proliferation, but increased adhesion to fibrillar collagen type I as compared to wild type SMC in vitro ${ }^{100}$.

Collagen organizational state can also influence SMC function. For example, Koyama et al. showed that SMC plated on polymerized collagen type I had decreased adhesive and proliferative properties whereas SMC plated on monomeric collagen type I had enhanced adhesive and proliferative capacities $^{101}$. Moreover, work by Orr et al. has shown that plating SMC on collagen IV upregulates SMC expression of contractile genes ${ }^{68}$. Lastly, Schlumburger et al. showed that treatment of pig aortic SMC with TGF $\beta$ in two dimensional culture produced an abundance of collagen that was did not incorporate well into an extracellular matrix ${ }^{96}$. In contrast, when these same pig aortic SMC were cultured in a three dimensional collagen lattice and treated with TGF $\beta$, less collagen was produced in comparison to the two dimensional culture, but there was more new collagen incorporated into the collagen lattice ${ }^{96}$. Taken together, in vitro interactions between SMC and collagens suggest that there is a complex, reciprocal relationship between the two molecules that will be important to consider when asking how SMC and collagens may be interacting in vivo. Yet despite the evidence that SMC can produce collagens and that collagens 
influence SMC function in vitro, there were previously no direct studies in vivo showing that SMC are collagen producing cells or that SMC produced collagens influence lesion development or indices of stability. The studies outlined in Chapter 2 of this dissertation represent the first studies that test if a SMC produced collagen influences atherosclerosis development.

\section{Overview and Rationale for the Study of Collagen Type XV, Alpha 1}

\section{(COL15A1).}

\subsection{The rationale for studying collagen type XV, alpha 1 (COL15A1).}

Fibrillar collagens form cellular scaffolds and localize mostly to the fibrous cap, where they have been credited with being important for plaque stability due to their resilience to mechanical forces ${ }^{46,79,80,102}$. It stands to reason that making changes to the fibrillar collagen scaffold such that it both fortifies the fibrous cap and lesion as well as promotes changes to SMC and macrophage function that enhance plaque stability would be ideal. Of interest, our lab identified a unique collagen, collagen type XV alpha 1, COL15A1, described in further detail below, that has been shown to be important in fibrillar collagen organization and maintenance of tissue structural integrity, is upregulated in human and mouse atherosclerotic lesions, and is a modifier of SMC proliferation and migration in vitro $^{21,23,103}$. We rationalized that studying SMC production of COL15A1 in the context of atherosclerosis would be a novel way to manipulate the fibrillar 
collagen matrix, specifically within the fibrous cap, and determine how and if a SMC-derived collagen can impact atherosclerotic lesion development.

\subsection{COL15A1 is a non-fibrillar, proteoglycan-like collagen molecule.}

COL15A1 was originally identified by Myers et al. in 1992 as a gene expressed within the human placenta ${ }^{22}$. COL15A1 is a unique non-fibrillar collagen with proteoglycan properties and a bow-like or pretzel shape ${ }^{104}$. COL15A1 is relatively large with the human COL15A1 gene spanning 127,079 base pairs (chromosome 9: 98943713-99070792) and 42 exons and the mouse Col15a1 gene spanning 105,153 base pairs (chromosome 4: 4720801247313165) and 38 exons $^{105}$. The COL15A1 protein itself is between 225-250 kilodaltons in size with the human protein containing 1388 amino acids and the mouse protein containing 1367 amino acids ${ }^{105,106}$. Structurally, the COL15A1 protein contains three distinct domains: an $\mathrm{N}$-terminal non-collagenous domain, a collagenous domain, and a C-terminal non-collagenous domain ${ }^{106}$. While the first 25 amino acids of COL15A1 is hypothesized to be a separate signaling domain, it remains to be proven whether there is a definitive function for this region ${ }^{107}$.

The N-terminal non-collagenous domain of COL15A1 contains a thrombospondin-1 motif and eight sites that can bind to glycosaminoglycan (GAG) side chains, which can increase the molecular weight of COL15A1 to around 400 kilodaltons ${ }^{23,24,107,108}$. COL15A1 contains a mix of mostly chondroitin sulfates and some heparan sulfates attached to these GAG sites which gives COL15A1 it's designation as a proteoglycan $23,24,104,108$. These GAG chains bind 
water which confers flexibility through their acting as a cushion to resist compressive forces $^{66,67}$. It is interesting to note that heparan sulfate GAG chains have been shown to bind vascular endothelial growth factor (VEGF) and fibroblast growth factor $(F G F)^{66,109,110}$. Moreover, chondroitin sulfate proteoglycans are hypothesized to bind lipids in the vessel wall which is important for initiating atherosclerosis lesion development ${ }^{111,112}$. However, there is currently no direct evidence that either the chondroitin or heparan sulfate GAG of COL15A1 bind to growth factors, cytokines, or lipids.

The collagenous domain of COL15A1 contains the classic Gly-X-Y repeats that define a collagen. These Gly-X-Y repeats are frequently interrupted by a variable number of amino acids; for example, human COL15A1 clones have been shown to contain between 8-13 interruptions ranging from 4-45 amino acids in size between Gly-X-Y repeats ${ }^{22,107,108}$. Notably, the first few interrupted regions of the collagenous domain contain GAG binding sites ${ }^{104}$ and the alpha 1 chain assembly of COL15A1 is mediated by two critical cysteine residues 231 amino acids apart within the collagenous domain ${ }^{24,107}$.

Lastly, the C-terminal domain of COL15A1 contains a C-terminal cleavage product originally called endostatin $\mathrm{XV}$ though later renamed restin. Restin is between $19-22$ kilodaltons in size with a high $61 \%$ protein sequence homology to endostatin, a C-terminal cleavage product of non-fibrillar COL18A1 $1^{106,113}$. However, the specific function for restin ${ }^{113-116}$ and how restin is cleaved from full length COL15A1 remains unknown. 


\subsection{COL15A1 functions as a fibrillar collagen organizer important for tissue integrity and can influence cell responses to injury and disease.}

COL15A1 has been found in vascular, neuronal, epithelial, and mesenchymal tissues ${ }^{23,24,117}$. COL15A1 is highly evolutionarily conserved and localizes to both the interstitial and basement membrane matrices ${ }^{22-24,117}$. Work by Amenta et al. has shown that COL15A1 links large collagen fibers and fibrils in vivo suggesting a role for COL15A1 in collagen and matrix organization and tissue stability ${ }^{23}$. In support of this, studies in global Col15a1 knockout mice have shown that global loss of COL15A1 results in cardiomyopathy and exercise induced skeletal myopathy ${ }^{25,26}$. Specifically, by five months of age, global Col15a1 knockout mice exhibit disorganized cardiomyocytes and tortuous heart microvessels ${ }^{25}$. Moreover, approximately $10 \%$ of the heart capillaries were ruptured $^{25}$. Skin and masseter muscle capillaries in these animals are also compromised as they have increased microvascular permeability ${ }^{25,118}$. Independently, the sciatic nerves of these mice occasionally display disrupted basement membranes and had disorganized C-fibers which carry sensory information ${ }^{119}$. Likewise in zebrafish, COL15A1 orthologs col15a1a and col15a1b are important in neuromuscular development and motor axon navigation ${ }^{120}$. In sum, these studies support a role for COL15A1 in tissue stability and matrix organization.

COL15A1 also affects cell function and acts as a physical barrier to cell invasion in injury and cancer. For example, Amenta et al. found through histological observations of stages of human breast ductal carcinoma that loss of 
COL15A1 from the in situ tumor ductal basement membrane precedes metastasis. Interestingly, subcutaneous injection of D98 human cervical carcinoma cells overexpressing full length human COL15A1 into mice led to a dose-dependent inhibition of tumor growth and increased survivability of the mice ${ }^{113,121}$. Similarly, overexpression of full length human COL15A1 in BxPC-3 pancreatic carcinoma cell lines results in an increase in cell adhesion, decrease in cell migration, and a decrease in epithelial to mesenchymal transition in vitro; this was mediated in part through binding E-cadherin and DDR1 and inhibiting signaling through DDR $1^{106}$. These experiments provide evidence for COL15A1 as a barrier to cancer cell motility. Intriguingly, in a model of kidney ischemiareperfusion injury, global Col15a1 knockout had a slight but significant reduction in neutrophil infiltration and increased expression in inflammatory cytokines monocyte chemoattractant protein 1 (MCP1) and tumor necrosis factor alpha (TNFa) expression at the site of injury ${ }^{122}$. Combined, these studies exemplify the breadth of COL15A1's influence and suggest the possibility that COL15A1 may modulate cell function and contribute to tissue stability in a cell and disease specific manner.

\subsection{COL15A1 is present and upregulated in atherosclerotic disease. Col15a1 expression modifies SMC function in vitro.}

As was mention above in section 3.1 , our lab identified COL15A1 as a novel molecule involved in SMC function and upregulated in atherosclerosis ${ }^{21}$. In a series of experiments in cultured human aortic SMC, COL15A1 was identified 
as an epigenetically regulated molecule that is hypomethylated with cell passage resulting in increased COL15A1 expression ${ }^{21}$. In addition, siRNA mediated knockdown of Col15a1 in cultured human aortic SMC showed a decrease in SMC proliferative and increase in SMC migratory capacity implicating COL15A1 as a modulator of SMC phenotype ${ }^{21}$.

COL15A1 expression was shown to be increased in both human and mouse atherosclerotic aorta indicating upregulation of COL15A1 is involved in atherogenesis ${ }^{21}$. Notably, Connelly et al. identified a single nucleotide polymorphism (SNP) rs4142986 (guanine (G) to cytosine (C)) that is associated with increased atherosclerotic disease risk in an aged ( $>55$ years) population ${ }^{21}$. Patient carriers of the risk allele (cytosine at rs4142986) had reduced expression of $C O L 15 A 1^{21}$. The cytosine risk allele creates a CpG site in the gene that can be methylated. Methylation of the C allele of rs4142986 correlated with increased Sudan $\mathrm{IV}^{+}$human aortic lesion burden in the seven patient samples assessed ${ }^{21}$. Taken together, we hypothesized that loss of SMC production of COL15A1 in atherosclerosis would result in disorganization of the fibrillar collagen matrix, specifically within the fibrous cap, which would consequently change the cell type accumulations within lesions such that lesions would have reduced indices of plaque stability. We therefore sought to answer the question of whether a SMCderived collagen, COL15A1, can impact atherosclerotic lesion development and stability. 


\section{Focus of the Dissertation}

The overall goal of my dissertation project was to determine the effect of SMC produced type XV collagen alpha 1 (COL15A1) on the development of advanced atherosclerotic lesions. COL15A1 is a non-fibrillar collagen that is upregulated in human and mouse atherosclerosis and has been previously shown to function as a link between large collagen fibers conferring extracellular matrix stability $21,22,24-26,104$. Atherosclerotic plaques are classically defined as stable if they contain an increased ratio of $\mathrm{ACTA}^{+}$presumed SMC to $\mathrm{CD}^{+} 8^{+}$ presumed macrophages within a collagen-rich fibrous $\operatorname{cap}^{2,7-9,31}$. SMC were believed to be the main producers of collagens, which provide tensile strength and stability to atherosclerotic plaques. However, no definitive in vivo evidence for SMC as collagen producers within lesions existed due to the fact that: 1) collagens are secreted molecules precluding easy determination of their cell source in vivo; and 2) recent lineage tracing studies by our lab ${ }^{14-16}$ and others ${ }^{17-}$ 20,60 that have shown that cell marker gene expression classically used to distinguish cell types within lesions are not specific. Consequently, unanswered questions remained in the field as to whether SMC are the primary source of collagens in vivo and if these collagens in turn impact atherosclerotic lesion development. We hypothesized that SMC-derived COL15A1 would play a critical role in plaque stability by promoting lesion collagen organization thereby impacting overall lesion development.

Studies in Chapter 2 outline our published results that are the first to provide definitive in vivo evidence that SMC can produce COL15A1 in 
atherosclerosis and that SMC production of COL15A1 plays a pivotal role in atherosclerotic lesion development. We developed a state of the art tamoxifen inducible SMC lineage tracing, SMC specific Col15a1 knockout mouse model that shows loss of COL15A1 in SMC leads to: 1) a drastic decrease in lesion and vessel size; 2) a marked reduction in lesion and medial collagen content and maturation likely contributing to reduced carotid artery vessel stiffness; 3) an overall reduction in total cells within the lesion with an increased proportion of SMC and decreased proportion of macrophages; and 4) a significant decrease in SMC proliferation within lesions as compared to wild type controls. Moreover, we provide evidence that loss of SMC-derived COL15A1 leads to global dysregulation of pathways involving nearly all immune cell types and inhibition of various pro-atherogenic inflammatory pathways. Combined, the data in this dissertation represent the first direct evidence that a SMC-derived collagen, COL15A1, can drastically impair atherosclerotic lesion development. Finally, Chapter 3 encompasses conclusions and potential future directions derived from discoveries put forth in this dissertation. 


\title{
CHAPTER 2
}

\section{Smooth muscle cell-specific deletion of Col15a1 unexpectedly leads to impaired development of advanced atherosclerotic lesions}

\begin{abstract}
Brittany G. Durgin ${ }^{1,2}$, Olga A. Cherepanova ${ }^{1,2}$, Delphine Gomez ${ }^{1,2}$, Themistoclis Karaoli $^{1}$, Gabriel F. Alencar ${ }^{1,3}$, Joshua T. Butcher ${ }^{1,2}$, Yu-Qing Zhou ${ }^{4}$, Michelle P. Bendeck $^{4}$, Brant E. Isakson ${ }^{1,2}$, Gary K Owens ${ }^{1,2 \star}$, Jessica J. Connelly ${ }^{1,5 *}$
\end{abstract}

${ }^{1}$ Robert M. Berne Cardiovascular Research Center, University of Virginia, Charlottesville, VA, USA

2.Department of Molecular Physiology and Biological Physics, University of Virginia, Charlottesville, VA USA

3.Department of Biochemistry and Molecular Genetics, University of Virginia, Charlottesville, VA USA

4. Department of Laboratory Medicine and Pathobiology, Ted Rogers Centre for Heart Research TBEP/ University of Toronto, Ontario, Canada

5.Department of Psychology, University of Virginia, VA, Charlottesville, VA USA

*authors contributed equally

Modified from the American Journal of Physiology: Heart and Circulatory Physiology (PMID:28283548) 


\section{Abstract}

Atherosclerotic plaque rupture with subsequent embolic events is a major cause of sudden death from myocardial infarction or stroke. Although smooth muscle cells (SMC) produce and respond to collagens in vitro, there is no direct evidence in vivo that SMC are a crucial source of collagens and that this impacts lesion development or fibrous cap formation. We sought to determine how conditional SMC specific knockout of collagen type XV (COL15A1) in SMC lineage tracing mice affects advanced lesion formation given: 1) we previously identified a Col15a1 sequence variant associated with age-related atherosclerosis; 2) COL15A1 is a matrix organizer enhancing tissue structural integrity; and 3) siRNA mediated Col15a1 knockdown increased migration and decreased proliferation of cultured human SMC. We hypothesized that SMCderived COL15A1 is critical in advanced lesions, specifically in fibrous cap formation. Surprisingly, we demonstrate that SMC specific Col15a1 knockout mice fed a Western diet for 18 weeks failed to form advanced lesions. SMC specific Col15a1 knockout resulted in lesions reduced in size by $78 \%$, with marked reductions in number and proliferating SMC, and lacked a SMC and ECM-rich lesion or fibrous cap. In vivo RNA-seq analyses on SMC Col15a1 knockout and wild type lesions suggest that a mechanism for these effects is through global repression of multiple pro-atherogenic inflammatory pathways involved in lesion development. These results provide the first direct evidence

that a SMC-derived collagen, COL15A1, is critical during lesion pathogenesis but 
contrary to expectations, its loss resulted in marked attenuation rather than exacerbation of lesion pathogenesis.

\section{Introduction}

Atherosclerosis is a pervasive, progressive disease and the leading cause of death in the Western world ${ }^{1,33,43,123}$. Enhancement of atherosclerotic plaque stability, specifically the strength of the fibrous cap, is of great clinical relevance as a majority of sudden deaths from myocardial infarction result from plaque rupture or erosion causing subsequent embolic events ${ }^{1,33,43,123}$. Both the cell composition and collagen deposition and organization of collagen within atherosclerotic lesions affect plaque formation and stability. Specifically, assessment of human plaque pathology has suggested that an increase in the ratio of $\mathrm{ACTA}^{+}$to $\mathrm{CD}^{+} 8^{+}$cells within lesions and a collagen-rich fibrous cap characterizes a stable plaque ${ }^{1,7,43}$. An organized fibrillar collagen-rich matrix (i.e. collagen types I and III) within the fibrous cap provides mechanical tensile strength and resistance to rupture ${ }^{46,75,77,79,124,125}$. In addition, multiple non-fibrillar collagen subtypes have been identified within the fibrous cap (types IV and VI) and throughout atherosclerotic plaques (types VIII, XV-XIX) ${ }^{69,93-95,126}$. Furthermore, conventional global knockout studies of specific non-fibrillar collagen subtypes VIII and XVIII in mouse models of atherosclerosis have provided evidence that collagens can contribute to plaque pathogenesis by impacting plaque size, cell composition and indices of stability ${ }^{94,95}$. However a major limitation in the use of conventional global collagen knockout mouse models is that it precludes determination of the cellular source of collagens within 
atherosclerotic lesions. Consequently, while there is substantial evidence establishing collagen content and organization as important in plaque and fibrous cap formation and stability, the identity of the major collagen producing cells within lesions remains unknown.

Nevertheless, it has been widely presumed in the field that smooth muscle cells (SMC) are the primary source of collagen within the lesion and fibrous cap. This assumption has been supported by several in vitro observations including that cultured SMC produce collagens in response to pro-atherogenic stimuli (e.g. TGF $\beta$, IL1 $\beta$, and oxidized phospholipids) ${ }^{11-13}$ and that collagens can influence SMC migration, proliferation, and adhesion ${ }^{13,127}$. However, definitive evidence for SMC as the in vivo source of collagen within lesions is lacking as: 1) collagen subtypes are secreted molecules making it difficult to ascertain their cell source; and 2) recent rigorous lineage tracing studies have shown that cell marker genes traditionally used to identify cells within atherosclerotic lesions are nonspecific $^{41,42}$. For example, cells other than SMC, such as macrophages ${ }^{18,128}$ and endothelial cells ${ }^{19}$, can activate traditional SMC marker genes within lesions ${ }^{14-}$ 16,20,42. Furthermore, recent rigorous SMC lineage tracing mouse studies by our lab ${ }^{14-16}$ demonstrated that $>80 \%$ of SMC within advanced lesions cannot be identified using traditional SMC lineage markers such as ACTA2 or TAGLN. Importantly, we also showed that nearly $40 \%$ of ACTA2 $^{-}$SMC have activated multiple markers of macrophages and mesenchymal stem cells and therefore have likely been misidentified in previous studies in the field ${ }^{15}$. Of most relevance to our understanding of the role of SMC-derived collagen production in human 
atherosclerosis, we presented compelling evidence that approximately $20 \%$ of cells previously identified as being macrophages within advanced human coronary artery lesions are actually of SMC rather than myeloid origin ${ }^{15}$. The converse is also true; Caplice et al. provided evidence based on analyses of advanced coronary artery lesions from subjects who had earlier undergone a cross gender bone marrow transplant, that a significant subset of ACTA2 $^{+}$ positive lesion cells are of myeloid origin ${ }^{17}$. Finally, a comprehensive study by Allahverdian et al. showed that nearly $50 \%$ of $\mathrm{CD}^{+} 8^{+}$cells within advanced human lesions are also $\mathrm{ACTA} 2^{+}$thus making it impossible to ascertain if these are SMC that have activated macrophage markers, macrophages that activated SMC markers, or neither ${ }^{58}$. Collectively, there are thus not only major ambiguities as to which cells within human and mouse lesions are SMC versus non-SMC, but nearly complete uncertainties as to what cells are the major source of lesion collagens.

Of particular importance, we previously identified a single nucleotide polymorphism (rs4142986) within the collagen type XV, alpha 1 gene (COL15A1) that is significantly associated with atherosclerosis in aged (>55 years) individuals $^{21}$. We also found that this polymorphism is associated with reduced COL15A1 expression levels, can be methylated, and its methylation is correlated with disease burden within human aortas ${ }^{21}$. COL15A1 is a non-fibrillar multiplexin collagen that connects large fibrillar collagens and organizes cellular matrices to enhance tissue structural integrity $23-26,103,107,119,121$. We discovered COL15A1 is upregulated in human and mouse atherosclerosis and siRNA mediated 
knockdown of Col15a1 in cultured human aortic SMC resulted in a decrease in SMC proliferation and an increase in SMC migration ${ }^{21}$. Taken together, we thus hypothesized that SMC-derived COL15A1 would play a critical role in plaque stability by promoting collagen organization within the fibrous cap of advanced lesions and that its loss would be associated with increases in overall lesion size but reductions in indices of plaque stability. However, completely contrary to expectations we found that SMC specific knockout of Col15a1 resulted in marked attenuation of lesion development with lesions in SMC specific Col15a1 knockout mice failing to advance beyond fatty streak formation in spite of 18 weeks of Western diet feeding. That is, production of COL15A1 by SMC had a major unanticipated novel atheropromoting role in mouse lesion development.

\section{Methods}

\section{Generation and validation of $\mathrm{Col} 15 \mathrm{at}{ }^{\mathrm{fl} / \mathrm{fl}}$ mice}

$\mathrm{C} 57 \mathrm{BL} / 6 \mathrm{~J} / \mathrm{N}-\mathrm{Col15a} 1^{\mathrm{tm} 1,2 \mathrm{Jjc}}\left(\mathrm{Col} 15 \mathrm{a} 1^{\mathrm{fl} / \mathrm{ft}}\right)$ mice were generated by TransViragen Inc. Excision of Col15a1 exons 1 and 2 was previously shown to be sufficient for gene knockout ${ }^{26}$. We therefore targeted these exons to generate Col15a $1^{\text {fl/fl }}$ mice as outlined in Figure 1 . In brief, the gene targeting cassette (Figure 1A) was transfected into $\mathrm{C} 57 \mathrm{BL} / 6 \mathrm{~N}$ embryonic stem cells, selected for neomycin resistance, and subsequently injected into albino C57BL/6J blastocysts (Jackson Labs 000058) to generate chimeras on a C57BL/6 background (Figure 1B). Chimeras were crossed to congenic C57BL/6J FLPe deleter mice

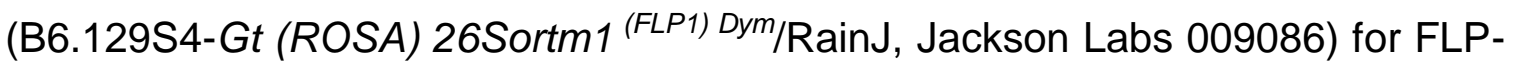
FRT mediated excision of the neomycin resistance component (Figure 1C). 
Female Col15a ${ }^{f / W t}$ mice were initially crossed with congenic C57BL/6 Myh11-CreER ${ }^{\mathrm{T} 2}$ mice on an $\mathrm{Apoe}^{-/-}$background ${ }^{129}$ resulting in generation of SMC specific Col15a1 floxed mice (Col15a1 ${ }^{f / f l} M^{f}$ Mh11-CreER ${ }^{\mathrm{T} 2}, \mathrm{Apoe}^{-/-}$or shorthand SMC Col15a1 $\left.1^{f / f t}, A p o e^{-/}\right)$. Female Col15a $1^{f / f t}, A p o e^{-/-}$animals were then crossed to Myh11-CreER ${ }^{\text {T2 }}$ ROSA26-STOP floxed eYFP Apoe ${ }^{--}$mice described previously ${ }^{15,16}$ to generate Col15a $1^{\text {fl/fl }}$ and Col15a ${ }^{\text {wtwt }}{ }^{\text {Myh11-CreER }}{ }^{\text {T2 }}$ ROSA26-STOP flox eYFP $A p o e^{-/-}$, which provide the added benefit of SMC specific lineage tracing $\left(\mathrm{YFP}^{+}\right)$(Figure 1D). Mice are designated as "SMC YFP"/+

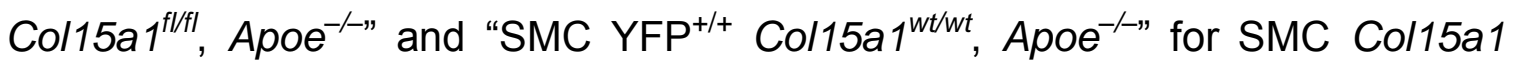
knockout and wild type respectively throughout the manuscript. Mice were genotyped as previously described ${ }^{16,129,130}$. Col15a1 genotyping primers are located in Table 1.

Myh11-CreER ${ }^{\mathrm{T} 2}$ resides on the $\mathrm{Y}$ chromosome therefore only male mice were used for our studies. Cre-Lox mediated knockout of Col15a1 was induced in 4 week old male mice following intraperitoneal injection of $1 \mathrm{mg}$ of tamoxifen (Sigma T-5648) per mouse per day for 7 days (Figure 1D). Col15a1 excision tissues ( $\mathrm{n}=9$ for aorta and $\mathrm{n}=8$ for carotid, heart, and kidney) were harvested and aortic SMC ( $\mathrm{n}=10$ pooled mouse thoracic aortas) isolated at 8 weeks of age. Col15a1 excision was assessed by Taqman Copy Number Variant Assay using a probe targeting exon 1 of Col15a1 (Life Technology, Mm00558775_cn) (Figures 2A \& B). No gross differences between SMC Col15a1 knockout and wild type were observed in any offspring. Information about the number of mice used for each analysis is indicated in the figure legends and throughout the detailed 
methods. Animal usage for these experiments was approved by the University of Virginia Animal Care and Use Committee.

\section{Animal diet and tissue processing}

At 6 weeks of age, mice were placed on a Western diet consisting of $21 \%$ milk fat and $0.15 \%$ cholesterol (TD.88137 Harlan Teklad) for 18 weeks. All SMC $\mathrm{YFP}^{+/+}$Col15a1 ${ }^{\text {wtwt }}, \mathrm{Apoe}^{-/}$and SMC $\mathrm{YFP}^{+/+} \mathrm{Col}_{15 a 1^{\Delta \Delta}}, \mathrm{Apoe}^{-/}$mice were given the same tamoxifen regimen. In addition, all SMC $\mathrm{YFP}^{+/+} \mathrm{Col15}^{{ }^{\mathrm{wt}} \mathrm{wt} \text {, }}$ $\mathrm{Apoe}^{-/}$and SMC $\mathrm{YFP}^{+/+} \mathrm{Col} 15 \mathrm{a} 1^{\Delta / \Delta}, \mathrm{Apoe}^{-/}$mice were given the same Western diet regimen with the exception of mice used for initial characterization of Col15a1 excision efficiency (i.e. Figures 2A \& B). Mice were euthanized after a 4 hour fast via carbon dioxide asphyxiation. Blood was collected and fasting plasma cholesterol and triglyceride level analyses were conducted by the University of Virginia Clinical Pathology Laboratory. Following euthanasia by carbon dioxide asphyxiation, mice were perfused with $10 \mathrm{~mL}$ phosphate buffered saline (PBS) and $10 \mathrm{~mL} 4 \%$ paraformaldehyde via the left ventricle. Brachiocephalic arteries (BCA) were dissected, post-fixed overnight in $4 \%$ paraformaldehyde, and paraffin embedded. Paraffin embedded BCA were sectioned serially at $10 \mu \mathrm{m}$ thickness from aortic arch to right subclavian artery for analysis.

\section{Immunohistochemical and immunofluorescent analysis}

Murine tissues

Modified Russell-Movat staining was conducted for vessel morphometry at three different locations on the BCA of SMC YFP ${ }^{+/+} \mathrm{Col}_{15 a 1^{\text {wt }} \text { wt }}, \mathrm{Apoe}^{-/-}(\mathrm{n}=11)$ 
and SMC $\mathrm{YFP}^{+/+}$Col15a1 ${ }^{f / f l}, A$ Apoe ${ }^{-/}(\mathrm{n}=13)$ mice. Immunohistochemical staining using an antibody specific for COL15A1 (HPA017913, Sigma Prestige, $0.48 \mu \mathrm{g} / \mathrm{mL}$ ) visualized with 3'3 diaminobenzidine (DAB, Acros Organic) was performed at one location of the BCA of SMC YFP ${ }^{+/+}$Col15a $1^{\text {wt } / w t}, A_{p o e^{--}}(\mathrm{n}=$ 11) and SMC YFP ${ }^{+/+}$Col15a1 $1^{f / f l}$, Apoe $^{-/}(\mathrm{n}=13)$ mice. COL15A1 staining was used to confirm Col15a1 knockout in the SMC-rich media and assess lesion COL15A1 content. Picrosirius Red staining was performed at one location of the BCA of SMC $\mathrm{YFP}^{+/+}$Col15a1 ${ }^{\text {wtwt }}$, Apoe $^{-/}(\mathrm{n}=11)$ and SMC $\mathrm{YFP}^{+/+} \mathrm{Col}_{15 a 1} 1^{f / / f t}$, Apoe $^{-/}(\mathrm{n}=13)$ mice to measure collagen content and maturation via fiber birefringence under polarized light. Immunofluorescent staining of BCA from

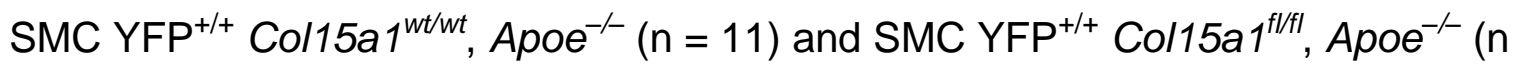
= 13) mice were done with primary antibodies specific for GFP (Abcam ab6673, 4 $\mu \mathrm{g} / \mathrm{mL}$ ), LGALS3 (Cedarlane CL8942AP, $0.2 \mu \mathrm{g} / \mathrm{mL}$ ), MKi67 (Abcam ab15580, 4 $\mu \mathrm{g} / \mathrm{mL}$ ), CASP3 (Cell Signaling 9661S, $0.84 \mu \mathrm{g} / \mathrm{mL}$ ), and DAPI (ThermoFisher Scientific D3571, $0.05 \mathrm{mg} / \mathrm{mL}$ ) as well as with a conjugated ACTA2-Cy3 (SigmaAldrich C6198; 1:500) antibody. Secondary antibodies for immunofluorescence included the following: donkey anti-goat 488 (Invitrogen A11055; 1:100), donkey anti-goat 647 (Invitrogen A21447; 1:100), donkey anti-rat Dylight 650 (Abcam ab102263; 1:100), donkey anti-rat Dylight 550 (Abcam ab102261; 1:100); donkey anti-rabbit 488 (Invitrogen A21206; 1:100). The number of locations assessed for each immunofluorescent primary antibody staining combination is indicated within the figure legends.

Human tissues 
De-identified coronary artery specimens from coronary artery bypass graft (CABG) patients were paraformaldehyde fixed, paraffin embedded, and sectioned at $5 \mu \mathrm{m}$ thickness. Arteries from 6 separate patients with advanced ( $>50 \%$ occluded) atherosclerotic lesions were selected. Two serial sections were stained. One section was stained using an antibody specific for COL15A1 (HPA017913, Sigma Prestige $0.48 \mu \mathrm{g} / \mathrm{mL}$ ) and visualized with $3^{\prime} 3$ diaminobenzidine (DAB, Acros Organic). The sequential section was stained with CD68 (Santa Cruz sc20060 KP1 clone, $2 \mu \mathrm{g} / \mathrm{mL}$ ), in conjunction with a secondary goat anti-mouse 647 antibody (Invitrogen A21236; 1:100), and ACTA2-Cy3 (Sigma-Aldrich C6198; 1:500) for analysis via immunofluorescence. The institutional review board of the University of Virginia approved use of these specimens.

Species matched immunoglobulins were used as a negative control for antibody staining in both human and murine tissues.

\section{Sudan IV analysis}

The thoracic aorta through the iliac bifurcation was isolated from SMC $\mathrm{YFP}^{+/+}$Col15a1 ${ }^{\mathrm{wt} / \mathrm{wt}}, \mathrm{Apoe}^{-/}(\mathrm{n}=9)$ and SMC YFP ${ }^{+/+} \mathrm{Col}_{15 a 1^{f / f l}}, \mathrm{Apoe}^{-/-}(\mathrm{n}=6)$ mice for en face Sudan IV staining analysis to assess aortic disease burden. The aorta was cleaned of periadventitial fat, dehydrated in $70 \%$ ethanol for 5 minutes, stained with Sudan IV (1g Sigma S-4261 diluted in $100 \mathrm{~mL}$ of $70 \%$ ethanol and $100 \mathrm{~mL}$ of $100 \%$ acetone) for 6 minutes, differentiated in $80 \%$ ethanol for 3 minutes, and stored in PBS at $4^{\circ} \mathrm{C}$.

\section{Image acquisition and analysis}


Movat, Sudan IV, and COL15A1 stained human and murine arteries were imaged using a Zeiss Axioskope2 microscope fitted with an AxioCamMR3 camera. Image acquisition was performed with AxioVision40 V4.6.3.0 software (Carl Zeiss Imaging Solution). Digitized images of Movat, Sudan IV, and COL15A1 stained tissues were all analyzed directly using Image Pro Plus Software 7.0 (Media Cybernetics Inc.). Areas of interest were drawn within the software to delineate external elastic lamina (EEL), inner elastic lamina (IEL), and lumen area with media and lesion areas extrapolated from these measurements. Positive staining color was selected at a pixel level and defined using a color cube based method.

To determine fibrillar collagen content, images of BCA sections stained with Picrosirius Red were taken on an Olympus BX51 under a polarized lens. Digitized images were analyzed directly using Image Pro Plus Software 7.0 (Media Cybernetics Inc.) in the same manner as Movat, Sudan IV and COL15A1 stained tissues.

To determine fibrillar collagen maturation, the Picrosirius red stained slides were assessed using quantitative polarization microscopy and an LCPolScope (Perkin Elmer). This system measures birefringence retardance of a sample in an orientation-independent manner and uses a polarization algorithm to compile images of the fibrillar organization of tissues. In the PolScope images, the color of each pixel is proportional to the amount of birefringent fibrillar collagen present in the tissue, as indicated on the spectrum key below the image, with blue representing less and red representing greater retardance. The sum of 
birefringence retardance was measured across the entire lesion and/or medial cross-sectional area and divided by the total area of lesion and/or media to obtain average retardance per pixel in each layer for each sample.

Immunofluorescent stained murine BCA were imaged using a Zeiss LSM700 confocal microscope. Zen 2009 Light Edition Software (Zeiss) was used to acquire a series of eight z-stack images at $1 \mu \mathrm{m}$ intervals. Careful analysis of each z-stack image was performed using Zen 2009 Light Edition Software (Zeiss) to assess co-localization of markers within a single cell (DAPI ${ }^{+}$nucleus) throughout the entire lesion. Images are maximum intensity projections for the confocal z-stacks. For immunofluorescent imaging of human coronary arteries, an Olympus IX51 microscope fitted with an Olympus XM10 camera was used with image acquisition performed using Olympic standard cellSENS software.

\section{Pressure Myography}

The right carotid artery region just above the subclavian artery was harvested from 18 week Western diet fed SMC Col15a1 $1^{\text {wtwt }}$, Apoe $^{-/-}(\mathrm{n}=6)$ and SMC Col15a1 ${ }^{\Delta / \Delta}$, Apoe $^{-/}(n=5)$ mice. Vessels were cannulated on glass micropipettes and secured with a 10-0 nylon monofilament suture to the arteriograph. Vessels were equilibrated at $90 \mathrm{mmHg}$ for approximately 30 minutes in Krebs-HEPES containing $118.4 \mathrm{mM} \mathrm{NaCl}, 4.7 \mathrm{mM} \mathrm{KCl}, 1.2 \mathrm{mM}$ $\mathrm{MgSO}_{4}, 4 \mathrm{mM} \mathrm{NaHCO} 3,1.2 \mathrm{mM} \mathrm{KH}_{2} \mathrm{PO}_{4}, 2 \mathrm{mM} \mathrm{CaCl}$, $10 \mathrm{mM}$ HEPES, $6 \mathrm{mM}$ glucose, and supplemented with 1\% BSA before assessment of active tone ${ }^{131}$. Pressure was subsequently dropped to $20 \mathrm{mmHg}$ and increased in 5 minute intervals (or until plateau was achieved) to $180 \mathrm{mmHg}$ in $20 \mathrm{mmHg}$ increments. 
Internal diameters were measured using digital calipers under a Danish Myotechnology microscope (DMT) using DMT vessel acquisition suite. Carotids were then incubated in calcium free Krebs-HEPES containing 2 mM EGTA and $10 \mu \mathrm{M}$ sodium nitroprusside buffer for assessment of passive tone. The pressure curve was then repeated for passive tone.

\section{Isolation of aortic SMC}

Mouse aortic SMC were isolated as previously described ${ }^{14,15}$. Briefly, after tamoxifen injections at 4 weeks of age, thoracic aortas from 10 individual SMC $\mathrm{YFP}^{+/+} \mathrm{Col} 15 \mathrm{a} 1^{\mathrm{\Delta} / \Delta}, \mathrm{Apoe}^{-/}$mice were harvested at 6 weeks of age. Aortas were cleaned of periadventitial fat and enzymatically digested in a mix of $1 \mathrm{mg} / \mathrm{mL}$ collagenase II, $1 \mathrm{mg} / \mathrm{mL}$ soybean trypsin inhibitor, $0.744 \mathrm{units} / \mathrm{mL}$ elastase (all from Worthington Biochemical Corp.) in Hank's balanced salt solution (HBSS) for $10 \mathrm{~min}$ at $37{ }^{\circ} \mathrm{C}$ in a $5 \% \mathrm{CO}_{2}$ incubator. The adventitia was then carefully removed using autoclaved sterilized tools and endothelial cells gently scraped off the luminal surface. The 10 aortas were then pooled and cut into approximately $0.5 \mathrm{~mm}$ pieces, placed in enzyme, and incubated for an hour to dissociate the SMC. SMC were then cultured in $20 \%$ serum containing media (DMEM/F12 (Gibco), fetal bovine serum (Hyclone), $100 \mathrm{U} / \mathrm{mL}$ penicillin/streptomycin (Gibco), and $1.6 \mathrm{mM} / \mathrm{L}$ L-glutamine (Gibco)) and plated at a high density to ensure cell recovery. SMC were changed to $10 \%$ serum-containing media after two passages. Flow cytometry analysis for endogenous YFP (data not shown) and Taqman Copy Number Assay for exon 1 of Col15a1 (Figure 2A) were conducted to confirm isolation of Col15a1 knockout SMC. 


\section{RNA-seq analysis}

The BCA, aortic arch, and carotid arteries from 18 week Western diet fed SMC YFP ${ }^{+/+}$Col15a1 ${ }^{\Delta / \Delta}$, Apoe $^{-/}(\mathrm{n}=3)$ and SMC YFP ${ }^{+/}$Col15a1 $^{\text {wtwt }}$, Apoe $^{-/}(\mathrm{n}$ = 3) mice were flash frozen in liquid nitrogen for RNA-seq analysis. Total RNA was extracted from arteries using Trizol RNA extraction per manufacturer's protocol (Invitrogen). RNA library and deep sequencing was performed by HudsonAlpha Institute for Biotechnology according to Illumina RNA Seq kit instructions with ribosomal reduction and strand specificity. Agilent 2100 Bioanalyzer and Kapa Library Quantification Kit (Kapa Biosystems) were used for quality control and quantification of the RNA and RNA library according to the manufacturer's protocol. Libraries were sequenced with the Illumina HiSeq 2000 (2 x 100bp, 25 million reads).

100 nucleotide paired-end reads were mapped to the $\mathrm{mm} 9$ reference genome using STAR software version $2.4^{132}$. A table of gene counts was generated using FeatureCounts in the Subread package ${ }^{133}$. DESeq2 Bioconductor R package ${ }^{134}$ was used to identify differentially expressed genes using Benjamini-Hochberg procedure to adjust $P$ values. GENCODE/Ensembl gene ID mapping to known genes were used, whereas those mapping to predicted genes were excluded. We performed Ingenuity Pathway Analysis ${ }^{135}$ on all differentially expressed genes to identify dysregulated pathways between SMC YFP ${ }^{+/}$Col15a1 $^{\Delta / \Delta}$, Apoe $^{-/}$and SMC YFP ${ }^{+/}$Col15a $1^{\text {wtwt }}$, Apoe ${ }^{-/}$lesions. We performed an Upstream Regulator Analysis to predict the biological factors (e.g. gene, small molecule, transcription factor) most likely to be driving the 
differences in gene expression between SMC $\mathrm{YFP}^{+/+} \mathrm{Col15a1}^{\Delta / \Delta}, \mathrm{Apoe}^{-/}$and SMC $\mathrm{YFP}^{+/+}$Col15a1 ${ }^{\text {wtwt }}$, Apoe ${ }^{-/-}$lesions. Upstream Regulator Analysis ${ }^{135}$ was done on the top approximately 3,000 differentially expressed genes with a log fold change of \pm 0.30 from the RNA-seq as this was the highest computational capacity of the program. The RNA-seq data is available at the NCBI Gene Ontology Omnibus (GEO) database under GEO accession number GSE94661.

\section{Statistical Analysis}

Statistics were performed using GraphPad Prism Version 6 software. Normality of the data was determined via Kolmogorov-Smirnoff normality test. In the case of Movat staining to ascertain overall morphometric parameters between SMC $\mathrm{YFP}^{+/+}$Col15a1 ${ }^{\Delta / \Delta}, \mathrm{Apoe}^{-/}$and SMC YFP ${ }^{+/+}$Col15a1 ${ }^{\mathrm{wt} / \mathrm{wt}}, \mathrm{Apoe}^{-/-}$ animals, a two-way ANOVA with repeated measures was performed to assess the consistency of the phenotype across the BCA. In all other cases where multiple BCA locations were analyzed, the values across each location were averaged for each individual mouse in the analysis and an individual comparison analysis was subsequently conducted comparing SMC $\mathrm{YFP}^{+/+} \mathrm{Col}_{15 a 1^{1 / \Delta}}, \mathrm{Apoe}^{-}$ ${ }^{-}$and SMC $\mathrm{YFP}^{+/+}$Col15a1 ${ }^{\text {wtwt }}$, Apoe ${ }^{-/}$mice. For individual comparisons of normally distributed data, unpaired two-tailed $t$-tests were performed with Welch's correction applied only in cases of unequal variance between groups. Mann Whitney $U$ tests were conducted if data were non-normally distributed. The statistical tests used for each data set are detailed in the figure legends. 


\section{$\underline{\text { Results }}$}

\section{SMC specific Col15a1 deletion resulted in marked reductions in the size of} brachiocephalic artery lesions

To determine the effect of SMC-derived COL15A1 on plaque pathogenesis, we created a Col15a ${ }^{f / f t}$ mouse and crossed it with a previously described tamoxifen inducible SMC lineage tracing, Apoe ${ }^{-/}$mouse (Myh11CreER $^{\text {T2 }}$ ROSA floxed STOP eYFP $\left.A p o e^{-/-}\right)^{14-16,129}$ to generate a SMC specific conditional Col15a1 knockout SMC-lineage tracing, Apoe ${ }^{-/}$mouse $\left(\mathrm{SMC} \mathrm{YFP}^{+/+}\right.$ Col15a1 $\left.{ }^{\Delta / \Delta}, A p o e^{--}\right)($Figure 1). After tamoxifen treatment, we placed six week old SMC $\mathrm{YFP}^{+/+}$Col15a ${ }^{\text {wtwt }}, \mathrm{Apoe}^{-/}$and SMC YFP ${ }^{+/+} \mathrm{Col}_{15 a 1^{\Delta / \Delta}}, A p o e^{-/}$mice on a Western diet for 18 weeks and isolated the brachiocephalic arteries (BCA) and aortas to characterize advanced atherosclerotic lesion development. PCR genotyping of DNA from whole aortic and carotid samples from SMC $\mathrm{YFP}^{+/+}$ Col15a1 $1^{\Delta / \Delta}$, Apoe ${ }^{-/}$mice showed $50 \%$ and $55 \%$ recombination efficiency, respectively, following tamoxifen treatment, which when corrected for the approximate $>50-60 \%$ SMC composition ${ }^{15}$ of these samples equates to $>95 \%$ recombination efficiency within SMC (Figures 2A \& B). Consistent with these data, we observed nearly $100 \%$ recombination efficiency in cultured SMC isolated from SMC $\mathrm{YFP}^{+/+} \mathrm{Col}_{15 a 1^{\Delta / \Delta}}, \mathrm{Apoe}^{-/}$mice (Figure 2A). Similarly, results of COL15A1 immunostaining of BCA sections from SMC $\mathrm{YFP}^{+/+}$Col15a1 ${ }^{\text {wtwt }}$, $\mathrm{Apoe}^{-/}$and SMC $\mathrm{YFP}^{+/+} \mathrm{Col}_{15 a 1^{\Delta \Delta}}, \mathrm{Apoe}^{-/}$mice showed a 95\% knockout efficiency within medial SMC (Figures 2C \& D). In addition, these results showed 
a $71 \%$ reduction in COL15A1 within lesions indicating that SMC are a major, though not exclusive, source of COL15A1 in atherosclerotic lesions.

Given that COL15A1 is involved in fibrillar collagen organization, can influence mechanical stability, and our prior observations of an association between a COL15A1 sequence variant and age-related human atherosclerosis $21,23,25,26,119$, we predicted that SMC Col15a1 knockout would result in larger lesions with characteristics associated with less stable lesions. Surprisingly, however, we found that SMC $\mathrm{YFP}^{+/+} \mathrm{Col15a1}^{\mathrm{\Delta \Delta}}, \mathrm{Apoe}^{-/}$mice exhibited a drastic, unexpected $78 \%$ decrease in BCA lesion size as compared to SMC $\mathrm{YFP}^{+/+}$Col15a1 ${ }^{\text {wtwt }}$, Apoe ${ }^{-/}$mice (Figures 3A \& B, [top left panel]). Furthermore, SMC $\mathrm{YFP}^{+/+} \mathrm{Col}_{15 \mathrm{a}}{ }^{\Delta \Delta}, \mathrm{Apoe}^{-/-} \mathrm{BCA}$ also had significantly smaller overall vessel size, as quantified by external elastic lamina area (Figure 3B, [top right panel]) and media area (Figure 3B, [bottom left panel]), but showed no difference in lumen area (Figure 3B, [bottom right panel]). This suggests that

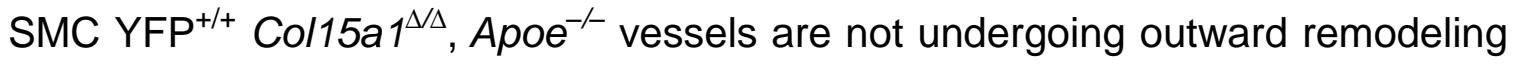
likely due to reduced necessity as a result of small plaque burden. Interestingly, en face luminal Sudan IV staining for lipids within the thoracic and abdominal aorta showed no differences in overall atherosclerotic disease burden between SMC $\mathrm{YFP}^{+/+} \mathrm{Col}_{15 a 1^{\Delta \Delta}}, \mathrm{Apoe}^{-/}$and SMC $\mathrm{YFP}^{+/+} \mathrm{Col}_{15 a 1^{\mathrm{wt}} \mathrm{wt}}, \mathrm{Apoe}^{-/}$mice (Figures $3 \mathrm{C} \& \mathrm{D}$ ). This suggests that while both SMC $\mathrm{YFP}^{+/+} \mathrm{Col15a1}^{\mathrm{\Delta \Delta}}, \mathrm{Apoe}^{-/-}$ and SMC YFP ${ }^{+/+}$Col15a $1^{\text {wtwt }}, \mathrm{Apoe}^{-/}$mice have equivalent atherosclerotic lesion burden, SMC $\mathrm{YFP}^{+/+} \mathrm{Co}_{115 a 1^{1 / \Delta}}, \mathrm{Apoe}^{-/}$develop drastically smaller lesions as

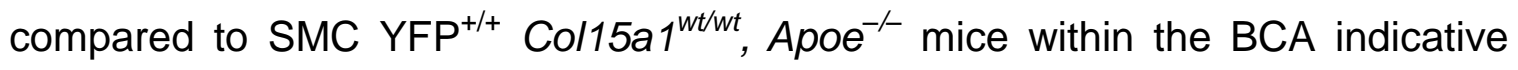


that SMC-derived COL15A1 is an essential component for lesion development and progression. Notably, no differences in body weight or fasting cholesterol and triglycerides were found between groups (Table 2). Taken together these data provide the first direct in vivo evidence that a collagen, COL15A1, produced by a single cell type, SMC, plays a critical role in atherosclerotic lesion pathogenesis. Contrary to predictions, however, loss of SMC-derived COL15A1 resulted in markedly impaired rather than exacerbated lesion development.

SMC specific deletion of Col15a1 reduces brachiocephalic artery collagen content, collagen organization, and carotid artery stiffness

We next tested if collagen deposition is broadly affected in SMC $\mathrm{YFP}^{+/+}$ Col15a ${ }^{\Delta / \Delta}$, Apoe ${ }^{-/}$lesions. Analysis of Picrosirius red stained BCA demonstrated that SMC $\mathrm{YFP}^{+/+} \mathrm{Col}_{15 a 1^{\Delta \Delta}}, \mathrm{Apoe}^{-/}$mice had an impressive $82 \%$ reduction in lesion and significant $24 \%$ reduction in media collagen content as compared to SMC Col15a1 ${ }^{\text {wtwt }}$, Apoe $^{-/}$mice (Figures 4A-C). Moreover, SMC $\mathrm{YFP}^{+/+} \mathrm{Col} 15 \mathrm{a} 1^{\Delta \Delta}, \mathrm{Apoe}^{-/}$mice exhibited a reduced average birefringence retardance in both the media and lesion indicative of a less mature fibrillar collagen matrix in both regions as compared to SMC Col15a $1^{\text {wtwt }}, \mathrm{Apoe}^{-/}$mice (Figures 4D-F). Combined, these data provide evidence that SMC produced COL15A1 contributes to fibrillar collagen deposition and organization within atherosclerotic lesions.

To determine if these changes had consequences on vessel function, we analyzed active and passive vascular tone in the carotid arteries of 18 week Western diet fed SMC Col15a1 ${ }^{\text {wtwt }}, \mathrm{Apoe}^{-/}$and SMC Col15a1 ${ }^{\Delta \Delta}, \mathrm{Apoe}^{-/-}$mice. 
Carotid arteries just above the right subclavian artery bifurcation were isolated and cannulated on glass micropipettes and then equilibrated in Krebs-HEPESbuffered physiological salt solution. By our observation, this region of the common right carotid, which is directly adjacent to the BCA, in both SMC $\mathrm{YFP}^{+/+}$ $\mathrm{Col}_{15 a 1^{\mathrm{L} \Delta}}, \mathrm{Apoe}^{-/-}$and SMC YFP ${ }^{+/+}$Col15a1 ${ }^{\mathrm{wt} / \mathrm{wt}}, \mathrm{Apoe}^{-/-}$was nearly devoid of lesions even after 18 weeks of Western diet feeding. Vessels were then subjected to increasing transmural pressures and internal lumen diameters were quantified. These experiments were done in the presence or absence of calcium in order to assess spontaneous active and passive tone, respectively (Figures 4G \& H). Carotid arteries from SMC Col15a1 ${ }^{\Delta / \Delta}, A p o e^{-/}$mice showed no significant differences in active tone (Figure 4G) but did show very modest but significant increases in passive tone (Figure $4 \mathrm{H}$ ) consistent with a minor decrease in overall vessel stiffness. Taken together, it therefore stands to reason that the decrease in media collagen content and maturation as a result of SMC Col15a1 knockout likely contributed to the observed functional decrease in stiffness.

\section{Loss of COL15A1 in SMC alters the number and cell type composition of lesions}

To understand how SMC-derived COL15A1 impacts the cellular composition of lesions, we performed high-resolution z-stack confocal microscopy for YFP (lineage tracing mark for SMC) as well as for ACTA2 (smooth muscle alpha actin, a traditional SMC marker) and LGALS3 (MAC2 antigen, a traditional macrophage marker) (Figure 5A). Remarkably, lesions from 
SMC $\mathrm{YFP}^{+/+} \mathrm{Col}_{15 a 1^{\mathrm{N} \Delta}}, \mathrm{Apoe}^{-/}$mice exhibited a marked $55 \%$ decrease in the overall number of cells as compared to SMC YFP ${ }^{+/+} \mathrm{Col}_{15 a 1^{\text {wt/wt }},}, \mathrm{Apoe}^{-/}$mice (Figure 5A \& B [DAPI]). Notably, lesions from SMC YFP ${ }^{+/+} \mathrm{Col}_{15 a 1^{1 / \Delta}}, \mathrm{Apoe}^{-/-}$ mice had very few SMC-derived cells within lesions (Figure 5C, $\left[\mathrm{YFP}^{+} / \mathrm{DAPI}^{+}\right]$) and virtually completely lacked an $\mathrm{ACTA} 2^{+}$fibrous cap (see the ACTA2 channel in Figure 5A). In contrast, SMC $\mathrm{YFP}^{+/+}$Col15a1 $^{\mathrm{\Delta \Delta}}$, Apoe $^{-/}$mice had an increased proportion of macrophages (Figure 5C, $\left[\mathrm{YFP}^{-} \mathrm{LGALS3}^{+} / \mathrm{DAPI}^{+}\right]$) as compared to SMC $\mathrm{YFP}^{+/+}$Col15a1 ${ }^{\text {wtwt }}, \mathrm{Apoe}^{-/}$lesions. Therefore, although SMC $\mathrm{YFP}^{+/+} \mathrm{Col} 15 \mathrm{a} 1^{\Delta \Delta \Delta}, \mathrm{Apoe}^{-/}$animals have a marked reduction in total number of cells within lesions, the cells that make up the lesions consist proportionately of more macrophages $\left(\mathrm{YFP}^{-} \mathrm{LGALS3}^{+}\right)$and fewer SMC $\left(\mathrm{YFP}^{+}\right)$as compared to lesions from SMC $\mathrm{YFP}^{+/+}$Col15a1 ${ }^{\text {wtwt }}, \mathrm{Apoe}^{-/}$mice. Taken together, SMC $\mathrm{YFP}^{+/+} \mathrm{Col}_{15 \mathrm{a}} 1^{\mathrm{\Delta} \Delta}, \mathrm{Apoe}^{-/}$mice develop less advanced, fatty streak-like lesions that are macrophage-rich, SMC-poor, and lack fibrillar collagens as compared to SMC $\mathrm{YFP}^{+/+}$Col15a1 ${ }^{\text {wtwt }}$, Apoe $^{-/}$lesions.

Next, we analyzed whether cell density differed between SMC $\mathrm{YFP}^{+/+}$ Col15a1 ${ }^{\text {wtwt }}, \mathrm{Apoe}^{-/}$and SMC YFP ${ }^{+/+} \mathrm{Col}_{15 a 1^{\Delta / \Delta}}, \mathrm{Apoe}^{-/-}$mice. We found that when normalized to lesion area, there was a significant increase in cell density of $\mathrm{DAPI}^{+}, \mathrm{LGALS}^{+}$, and $\mathrm{ACTA}^{+}$cells but no change in $\mathrm{YFP}^{+}$cell density within $\mathrm{SMC} \mathrm{\textrm {YP } ^ { + / + } \text { Col15a1 }}{ }^{\mathrm{\Delta} \Delta}, \mathrm{Apoe}^{-/}$lesions as compared to SMC $\mathrm{YFP}^{+/+}$ Col15a ${ }^{\text {wtwt }}, A p o e^{-/}$lesions (Figure 5D). It is important to note that SMC $\mathrm{YFP}^{+/+}$ Col15a ${ }^{1 / \Delta}, A p o e^{-\alpha}$ animals contain much smaller, less complex lesions with a reduced frequency of acellular, necrotic core areas as compared to SMC $\mathrm{YFP}^{+/+}$ 


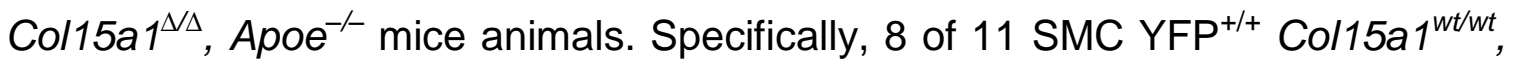
Apoe $^{-/}$animals in this analysis had large, established acellular necrotic cores while only 2 of $13 \mathrm{SMC} \mathrm{YFP}^{+/+} \mathrm{Col}_{15 a 1^{\Delta / \Delta}}, \mathrm{Apoe}^{-/}$animals contained any small, acellular necrotic core-like regions ( $P=0.01$, Fisher's Exact Test, data not shown). We also analyzed cell density as a function of the lesion area without inclusion of the acellular, necrotic core regions. Interestingly, we found that while the cell density of $\mathrm{DAPI}^{+}, \mathrm{LGALS}^{+}$, and $\mathrm{ACTA}^{+}$cells remained increased in SMC $\mathrm{YFP}^{+/+} \mathrm{Col}_{15 a 1^{\Delta \Delta}}, \mathrm{Apoe}^{-/}$compared to SMC $\mathrm{YFP}^{+/+}$Col15a1 ${ }^{\mathrm{wt} / \mathrm{wt}}, \mathrm{Apoe}^{-/-}$ lesions, the $\mathrm{YFP}^{+}$cell density was found to be significantly reduced as a result of SMC Col15a1 knockout (Figure 5E). This suggests that though SMC $\mathrm{YFP}^{+/+}$ Col15a1 ${ }^{\Delta / \Delta}, A p o e^{-/}$lesions overall appear to be more cell dense, there is a significant reduction in the proportion of SMC present within SMC $\mathrm{YFP}^{+/+}$

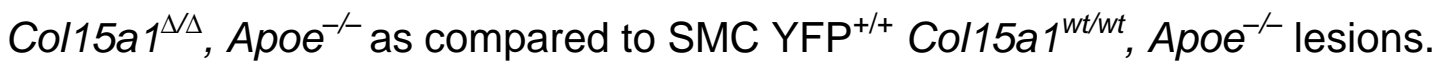

We then investigated the cells expressing traditional SMC marker gene ACTA2. Of interest, we observed no difference in the proportion of $\mathrm{ACTA}^{+}$cells per total lesion cells $\left(\mathrm{ACTA}^{+} / \mathrm{DAPI}^{+}\right)$between SMC $\mathrm{YFP}^{+/+}$Col15a1 ${ }^{\mathrm{wt} w t}, \mathrm{Apoe}^{-/-}$ and SMC $\mathrm{YFP}^{+/+} \mathrm{Col}_{15 a 1^{1 / \Delta}}, \mathrm{Apoe}^{-/}$lesions (Figure 6A). This result and the observation of increased $\mathrm{ACTA}^{+}$cell density in $\mathrm{SMC} \mathrm{YFP}^{+/+} \mathrm{Col} 15 \mathrm{a} 1^{\mathrm{N \Delta}}, \mathrm{Apoe}^{-/-}$ lesions differs from prior global collagen knockout studies where a decrease in $\mathrm{ACTA}^{+}$cells and $\mathrm{ACTA}^{+}$cell density within atherosclerotic lesions was consistently observed irrespective of the collagen type knockout ${ }^{93-95}$. However, if we divided the $\mathrm{ACTA} 2^{+}$cells within the lesions in our studies into SMC- and non SMC-derived, we found that there was a decrease in the proportion of $\mathrm{ACTA}^{+}$ 
SMC-derived cells $\left(\mathrm{YFP}^{+} \mathrm{ACTA} 2^{+} / \mathrm{DAPI}{ }^{+}\right)$and a near significant increase in the proportion of non SMC-derived $\mathrm{ACTA}^{+}$cells $\left(\mathrm{YFP}^{-} \mathrm{ACTA}^{+} / \mathrm{DAPI}^{+}\right)$within $\mathrm{SMC}$ $\mathrm{YFP}^{+/+} \mathrm{Col}_{15 a 1}^{\mathrm{L \Delta}}, \mathrm{Apoe}^{-/-}$lesions as compared to SMC $\mathrm{YFP}^{+/+}$Col15a1 ${ }^{\mathrm{wt} w t}$, $A_{p o e^{-/}}$lesions (Figure 6B). Moreover, no differences in the proportion of SMC expressing ACTA2 $\left(\mathrm{YFP}^{+} \mathrm{ACTA}^{+} / \mathrm{YFP}^{+}\right)$or $\mathrm{LGALS3}\left(\mathrm{YFP}^{+} \mathrm{LGALS3}^{+} \mathrm{YFP}^{+}\right)$ though a significant increase in the proportion of LGALS3 $^{+}$cells $\left(\mathrm{LGALS3}^{+} / \mathrm{DAPI}^{+}\right)$was observed between SMC $\mathrm{YFP}^{+/+} \mathrm{Col}_{15 a 1^{\Delta / \Delta}}, \mathrm{Apoe}^{-/}$and SMC $\mathrm{YFP}^{+/+}$Col15a1 ${ }^{\text {wt } / \text { wt }}$, Apoe ${ }^{-/}$lesions (Figures 6A \& C). SMC $\mathrm{YFP}^{+/+}$ Col15a1 $^{\Delta \Delta \Delta}$, Apoe ${ }^{-/}$mice therefore are still able to maintain $\mathrm{ACTA2}^{+}$cell contribution to the lesion but loss of SMC produced COL15A1 reduces the proportion of $\mathrm{ACTA}^{+} \mathrm{SMC}$ contribution as compared to wild type lesions.

To determine mechanisms by which loss of SMC-derived COL15A1 resulted in reduced SMC number within lesions, we performed fluorescence coimmunostaining for YFP, LGALS3, and MKi67 (a marker of proliferation) or YFP, LGALS3 and cleaved CASP3 (a marker of cell death). There was no difference in the overall amount of proliferating cells per total number of lesion cells $\left(\mathrm{MKi67}^{+} / \mathrm{DAPI}^{+}\right)$between groups (Figures $\left.7 \mathrm{~A}-\mathrm{C}\right)$. However, we observed a significant $85 \%$ reduction in the proportion of SMC proliferating $\left(\mathrm{YFP}^{+} \mathrm{MKi67}^{+} / \mathrm{DAPI}^{+}\right.$, Figures 7B, [yellow arrows], \& D) within SMC $\mathrm{YFP}^{+/+}$

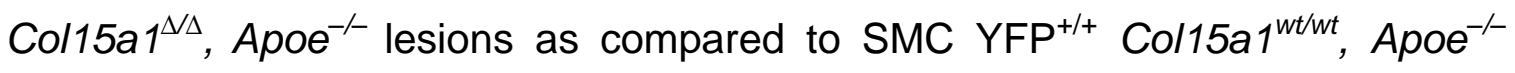
lesions. In contrast, there appeared to be a modest increase in proliferation of macrophages $\left(\mathrm{YFP}^{-} \mathrm{MKi}^{+} \mathrm{LGALS3}^{+} / \mathrm{DAPI}^{+}\right.$, Figures 7B, [white arrows], \& D) within SMC $\mathrm{YFP}^{+/+} \mathrm{Col}_{15 a 1^{\Delta \Delta}}, \mathrm{Apoe}^{-/}$lesions as compared to $\mathrm{SMC} \mathrm{YFP}^{+/+}$ 
Col15a ${ }^{\text {wtwt }}, A p o e^{-/}$lesions although this did not reach statistical significance (P $=0.06)$. SMC Col15a1 knockout thus appears to differentially impact SMC versus non-SMC proliferation within lesions. SMC $\mathrm{YFP}^{+/+} \mathrm{Col}_{15 a 1^{\mathrm{N}}}, \mathrm{Apoe}^{-/}$lesions also were nearly devoid of $\mathrm{CASP}^{+}$cells compared to their SMC $\mathrm{YFP}^{+/+}$ Col15a1 ${ }^{\text {wt }}$ wt,$A p o e^{-/}$counterparts (Figures $8 \mathrm{~A}-\mathrm{C}$ ) providing evidence that the reduction in overall cell number within $\mathrm{SMC} \mathrm{YFP}^{+/+} \mathrm{Col}_{15 a 1^{\Delta \Delta}}, \mathrm{Apoe}^{-/-}$lesions is not due to increased cell death and likely reflect the reduced lesion complexity observed as a consequence of SMC Col15a1 knockout.

\section{Loss of SMC-derived COL15A1 results in dysregulation of immune cell pathways important in driving lesion development}

The preceding results indicate that the reduced lesion size and complexity observed in SMC $\mathrm{YFP}^{+/+} \mathrm{Col}_{15 a 1^{1 / \Delta}}, \mathrm{Apoe}^{-/}$lesions is due in part to an overall decrease in the number of cells within the lesions including both SMC and macrophages. However, in aggregate these two cell types only account for about half of the overall reduction in cell number within lesions of the SMC $\mathrm{YFP}^{+/+}$ Col15a ${ }^{\Delta \Delta \Delta}, A p o e^{-/}$mice. To further understand the mechanism(s) by which loss of SMC-derived COL15A1 resulted in dramatic decreases in lesion size and complexity, we performed in vivo RNA-seq analysis on combined aortic arch, $\mathrm{BCA}$, and carotid artery tissue samples of 18 week Western diet fed SMC $\mathrm{YFP}^{+/+}$

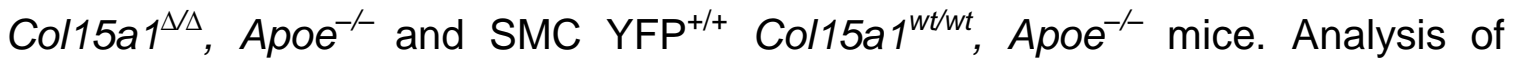
complex tissue samples consisting of multiple cell types is usually highly problematic in that it is nearly impossible to rigorously ascertain what changes are contributed by one cell type versus another. In addition, many differences 
may be lost due to inadequate sensitivity in detecting changes in relatively low abundance cell types and/or offsetting changes in gene expression in different cell types. However, an extremely powerful aspect of our approach is that we know that changes were initiated by loss of one gene, Col15a1, in one cell type, SMC. Of major interest, results of Ingenuity Pathway Analysis ${ }^{135}$ of the RNA-seq showed marked dysregulation of multiple immune cell pathways including dendritic cell maturation, altered $\mathrm{T}$ and $\mathrm{B}$ cell signaling, NF-kB signaling, granulocyte adhesion and diapedesis, and leukocyte extravasation signaling in response to SMC Col15a1 knockout (Figure 9A).

As there are myriad cell types present within atherosclerotic vessels, we are unable to make conclusions as to whether the pathway changes derived from this analysis are due to direct or indirect effects of SMC Col15a1 knockout. However, we employed a recently developed bioinformatics analysis program referred to as 'Upstream Regulator Analysis' ${ }^{135}$ to attempt to identify the upstream drivers responsible for differences between SMC $\mathrm{YFP}^{+/+} \mathrm{Col15a} 1^{\Delta / \Delta}$, $A p o e^{-/}$and SMC $\mathrm{YFP}^{+/+}$Col15a1 ${ }^{\text {wtwt }}, \mathrm{Apoe}^{-/}$lesions. That is, in our case this analysis takes the RNA-seq differential gene expression data derived from the

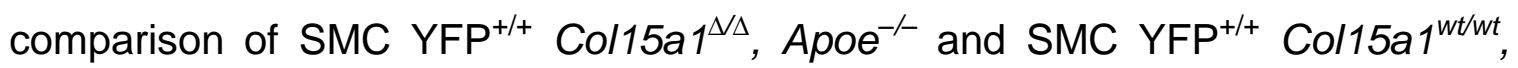
$A p o e^{-/}$lesions and integrates it with cause-effect relationships reported in the literature to attempt to predict master regulatory pathways (e.g. genes, transcription factors, small molecule driven pathways) most responsible for the difference in phenotype observed between SMC Col15a1 knockout and wild type lesions. Of major interest, these analyses identified significant repression of 
multiple factors as a result of SMC Col15a1 knockout including: lipopolysaccharide, tumor necrosis factor (TNF), transforming growth factor beta (TGF 31 ), interferon gamma (IFNG), colony stimulating factor 2 (CSF2), interleukin 6 (IL6), tretinoin (retinoic acid), interleukin 1 beta (IL1 $\beta$ ), phorbol myristate acetate, Erb-B2 receptor tyrosine kinase 2 (ERBB2), and genistein (Figure 9B). These results suggest that the mechanisms underlying the substantial decrease in lesion size and severity in our SMC Col15a1 knockout mice are extremely complex, but likely to involve rather profound global repression of multiple pro-inflammatory processes and immune cell responses. In addition, results of these analyses also identified a number of pathways that are

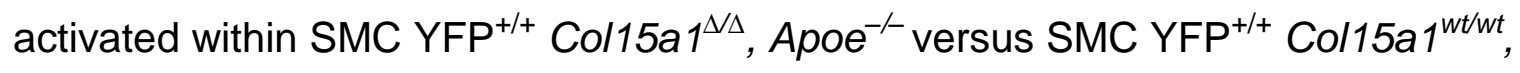
Apoe $^{-/}$lesions including dexamethasone, peroxisome proliferator-activated receptor gamma (PPARG), and rosiglitazone (a PPARG agonist) pathways (Figure 9B). Whereas these pathways can impact a wide range of cellular functions, the role of dexamethasone in atherosclerosis is unclear even though it is predominantly described as anti-inflammatory ${ }^{136,137}$. In addition, PPARG activation and the PPARG agonist rosiglitazone have been shown to be atheroprotective ${ }^{138-143}$. Taken together, our in vivo genomic analyses provide evidence that SMC-derived COL15A1 can positively impact a variety of proatherogenic processes, including immune cell activation, adhesion, migration, and cytokine expression, such that SMC-derived COL15A1 loss results in rather profound inhibition of lesion development and pathogenesis. 
COL15A1 is prevalent in ACTA2 $^{+}$regions within advanced human atherosclerotic plaques including the fibrous cap.

Given the importance of the fibrous cap in maintaining human plaque stability, we reasoned that identifying COL15A1 expression within human lesions would be important to our understanding of how COL15A1 may be contributing to plaque formation and stability. Coronary arteries with advanced lesions from six patients who underwent coronary artery bypass grafting (CABG) were stained for COL15A1 (Figure 10A). Serial slides were stained with the traditional macrophage marker CD68 and traditional SMC marker ACTA2 to provide a general characterization of the cell populations present within the lesion (Figure 10B). We found that COL15A1 is highly expressed throughout $\mathrm{ACTA}^{+}{ }^{+}$rich fibrous caps and within the plaque shoulder regions suggesting a role for COL15A1 in maintenance of overall plaque and fibrous cap stability (Figures 10C \& D). Interestingly, COL15A1 also localized to intraplaque microvessels which coincides with previous studies in mice showing COL15A1 expression within the microvasculature $^{25,26}$ (Figure 10D, [blue arrows]). This data therefore provides the first assessment of COL15A1 expression in advanced human lesions. In addition, the mouse and human data in this manuscript provide the first detailed assessment of COL15A1 expression within lesions and COL15A1 function in atherosclerotic lesion development. 


\section{Discussion}

Fibrillar collagens are known to localize to the fibrous cap and provide mechanical stability to atherosclerotic plaques ${ }^{8,43,77,144}$. COL15A1 serves as a linker of large fibrillar collagens providing structural integrity to collagen matrices $^{23}$. We therefore predicted that SMC-derived COL15A1 would have an atheroprotective role in stabilizing advanced atherosclerotic lesions through organizing and fortifying the ECM within the fibrous cap. Consistent with this hypothesis, we observed COL15A1 staining within the fibrous cap of advanced human lesions. Surprisingly, however, our data indicate that SMC Col15a1 knockout prior to the start of 18 weeks Western diet induced atherosclerosis led to the development of lesions $78 \%$ smaller than those of wild type controls. In addition, SMC Col15a1 knockout lesions exhibit characteristics consistent with those of fatty streaks; they are macrophage-rich, display a paucity of SMC and collagen, and are devoid of a fibrous cap and necrotic core. In stark contrast, wild type mice exhibited typical advanced BCA lesions including a SMC-rich, collagen-rich fibrous cap. Taken together, these results provide the first direct evidence that a collagen produced by SMC can impact plaque pathogenesis and that COL15A1 has a role in promoting lesion development.

The data presented herein suggest that mechanisms of SMC COL15A1 action in impairing lesion development are both diverse and complex. One such mechanism we observed is that loss of SMC-derived COL15A1 results in reductions in the overall numbers of SMC and macrophages within lesions. These findings are consistent with previous studies of conventional global 
Col15a1 knockout mice showing that global loss of COL15A1 from birth affects multiple cell types as these mice develop cardiovascular and skeletal myopathy ${ }^{25,26}$, impaired peripheral nerve maturation ${ }^{119}$, and in a model of renal ischemia reperfusion injury have reduced neutrophil infiltration but no change in macrophage $\left(\mathrm{F} 4 / 80^{+}\right)$infiltration ${ }^{122}$. Our work builds upon and advances this knowledge; we are the first to provide evidence that the loss of COL15A1 production from a single cell type, SMC, has a vast and varied effect on the function of multiple cell types during atherogenesis. Specifically, our RNA-seq analysis of BCA lesions indicates that the net effect of SMC Col15a1 knockout is the dysregulation of pro-inflammatory pathways associated with immune cells (i.e. T cells, B cells, macrophages, dendritic cells, granulocytes and leukocytes), endothelial cells, and fibroblasts. SMC Col15a1 knockout also results in an overall increase in lesion cell density but a decrease in the proportion of SMC and an increase in the proportion of macrophages populating the lesions as compared to wild type lesions. A second important role for COL15A1 is as a modulator of cell proliferation within lesions as loss of SMC-derived COL15A1 resulted in an $85 \%$ reduction in SMC proliferation and near significant increase in non SMC-derived macrophage proliferation within lesions. SMC-derived COL15A1 thus has a major influence on the function of nearly all cell types involved in lesion development. We propose that COL15A1 is an integral component within the lesion environmental milieu crucial in driving atherosclerotic plaque formation. Lastly, it is known that COL15A1 is an ECM organizing molecule $23-26,103,107,119,121$. Collagen types I and III are, to our 
knowledge, the most well-studied collagens in regards to atherosclerosis ${ }^{13,70,75-}$ 77. However, there are nearly thirty collagen types all of which may be influenced by loss of COL15A1 production exclusively by SMC. We therefore did not specifically measure collagens types I and III, but instead used Picrosirius red staining to evaluate the net changes in collagen content and maturation within the lesions. Notably, we observed that SMC Col15a1 knockout resulted in a $24 \%$ reduction in media and a drastic $82 \%$ reduction in lesion fibrillar collagen content, a reduced media and lesion collagen organization, as well as a functional increase in carotid artery elasticity. Likewise, we observed a $71 \%$ decrease in lesion COL15A1 expression with SMC Col15a1 knockout. Combined, this provides the first evidence that SMC are a major, though not sole, source of COL15A1 and likely collagens within lesions. We suspect that the effects on lesion development as a result of SMC Col15a1 knockout may therefore be due in part to impaired formation and organization of a three-dimensional matrix scaffold needed for SMC and other lesion cells to attach, migrate and proliferate into and within lesions. Importantly, together these data represent a significant advance in the field by providing the first direct in vivo evidence that a SMCderived collagen, COL15A1, directly influences plaque formation.

Prior to our studies, data from global knockout of non-fibrillar collagen types VIII and XVIII from birth and throughout lesion development provided evidence that collagens can have broad impacts on lesion development by observing changes in cellular and collagen content of lesions and in type XVIII knockouts specifically, changes in the size of lesions ${ }^{94,95}$. However, there are 
two inherent limitations in these prior global collagen knockout studies including: 1) the inability to determine the cellular source of collagen; and 2) global loss of the respective collagens from birth likely led to the activation of compensatory pathways that confound data interpretation. Notably, our studies and others in the field are also limited by the fact that collagens are extracellular proteins making it nearly impossible to determine if the observations made in association with manipulating collagen levels in vivo are the consequence of direct or indirect effects. In the case of COL15A1, this is further complicated by several unique properties of the N-terminal and C-terminal domains of COL15A1. First, the noncollagenous $\mathrm{N}$-terminal domain of COL15A1 contains chondroitin sulfate side chains similar to that of a proteoglycan ${ }^{24,107}$. There is evidence in the field that proteoglycans containing chondroitin sulfate side chains can bind apoB100 and LDL and is hypothesized to occur in the arterial wall contributing to early lesion development ${ }^{111,145,146}$. However, there is currently no evidence in the field that COL15A1 itself can directly bind lipids. Second, the C-terminus of COL15A1 contains a cleavage product called restin ${ }^{106}$. To our knowledge the role of restin in atherosclerosis has not been previously investigated and studies are conflicting over whether this molecule is involved in angiogenesis ${ }^{107,113,115,116,147}$. Given these results and those of our in vivo RNA-seq analyses, it seems likely that COL15A1 production by SMC is causing a number of direct and indirect changes that in aggregate result in profound inhibition of lesion development. Since in vitro cell culture approaches are unlikely to replicate the complex lesion environmental milieu, multicellularity, and extracellular architecture that exist 
within lesions in vivo, we took an alternative approach and performed an Upstream Regulator Analysis that focused on understanding the net effect of SMC-derived COL15A1 action on lesion development. These analyses identified SMC-derived COL15A1 dependent pathways that were highly significantly repressed which included several pro-inflammatory and immune cell response factors previously implicated in lesion development including lipopolysaccharides ${ }^{148}$, tumor necrosis factor $(\mathrm{TNF})^{149}$, transforming growth factor beta $(\mathrm{TGF} \beta)^{150}$, interferon gamma (IFNY) ${ }^{151}$, colony stimulating factor 2 (CSF2) and interleukin 1 beta (IL1 $\beta)^{152}$ in lesions from SMC Col15a1 knockout versus wild type mice. Whereas these data fail to provide direct insight as to how loss of SMC-derived COL15A1 impacts the multiplicity of cells within developing lesions, the strength of the data lies in that they provide insight as to possible rate limiting pathways essential for SMC COL15A1 dependent lesion development.

A critical question is to what extent our findings are relevant to human atherosclerosis. Of major interest, we provide evidence showing that COL15A1 is highly expressed within advanced human lesions, including plaque shoulders and caps, believed to be important in plaque stability. Moreover, our previous studies of COL15A1 in human atherosclerosis provided correlative evidence suggesting that a reduction in COL15A1 expression in patients carrying the risk allele may be associated with increased Sudan $\mathrm{IV}^{+}$aortic lesion burden. However, these human studies were based on a very small patient cohort and did not include direct characterization of indices of plaque stability. In addition, results of the present studies showing that SMC specific loss of Col15a1 prior to 
initiation of Western diet greatly attenuates lesion development, may not predict the role of this molecule in advanced lesions and/or if therapeutic targeting of COL15A1 is a viable means to promote plaque stabilization. To address these critical questions, future studies would need to include: 1) a large scale clinical study to assess if there is a correlation between COL15A1 levels within patient lesions and the incidence of S-T elevation mediated myocardial infarctions (STEMI) or stroke; and 2) experimental animal studies to test if reduction of COL15A1 levels after establishment of advanced atherosclerotic lesions has beneficial effects on lesion composition and indices of plaque stability. 
Figure 1

A) Col15a1 targeting vector

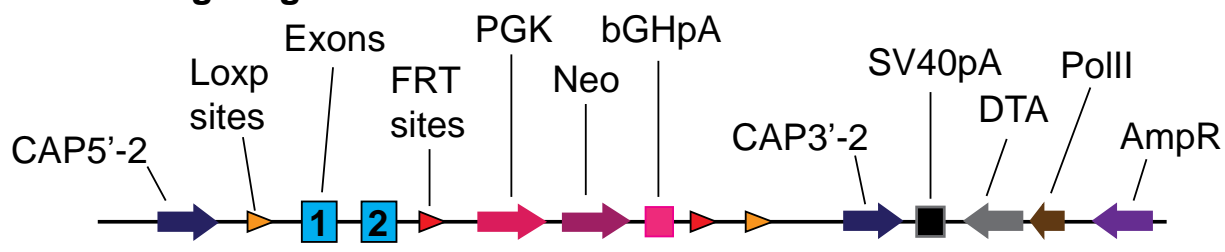

B) Col15a1 genome targeted insertion

5 '- probe

3'- probe

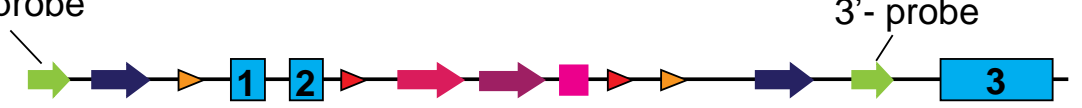

C) Cross Col15a1 chimera with Flpe mouse to generate Col15a $1^{1 / t / 1}$

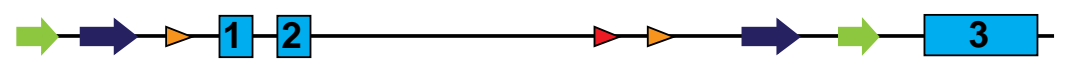

D) SMC specific Col15a $1^{\text {t/t/l }}$ SMC lineage tracing mice

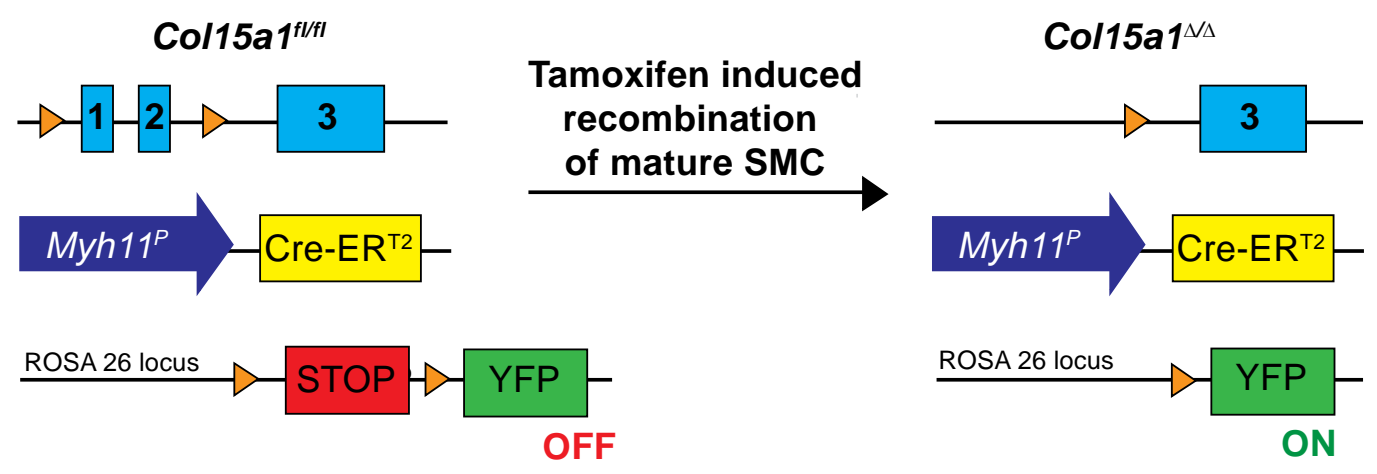


Figure 1: Generating Col15a1 ${ }^{f / f l}$ mice and SMC specific Col15a ${ }^{f / f l}$, SMC lineage tracing mice. (A) Schematic of Col15a1 targeting vector injected into embryonic stems cells. Vector insertion resulted in selective neomycin resistance of the embryonic stem cells. (B) Schematic of Col15a1 genome targeted insertion contained within chimera pups. (C) Schematic showing cross of Col15a1 chimera with Flpe recombinase mice results in excision of neomycin selectivity. This represents the Col15a1 locus in the animals used in our studies. (D) Schematic representation of SMC specific Col15a $1^{f / / f l}$ SMC lineage tracing mice before and after tamoxifen treatment. Myh11 ${ }^{P}$ is the Myh11 promoter. Cre-ER ${ }^{\top 2}$ is the estrogen receptor bound Cre recombinase. 
Figure 2

A)

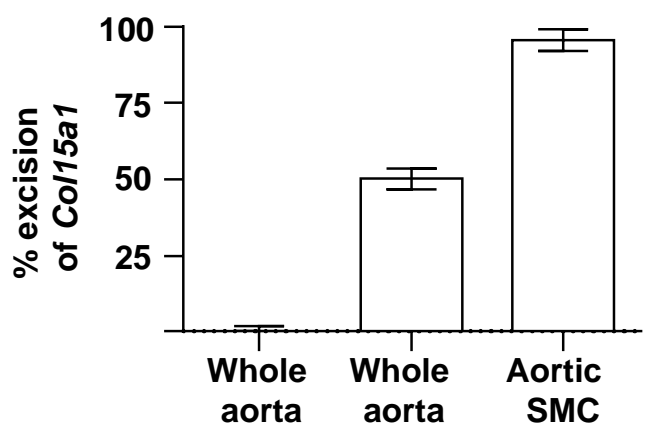

B)

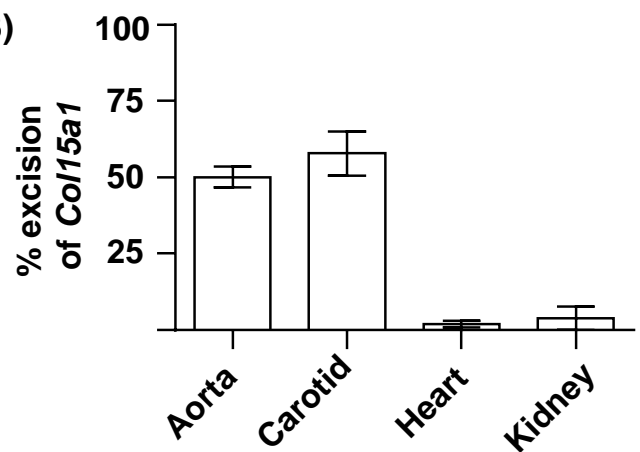

Tamoxifen $+$

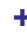

C) SMC YFP $+/+$ SMC YFP ${ }^{+/+}$

D)
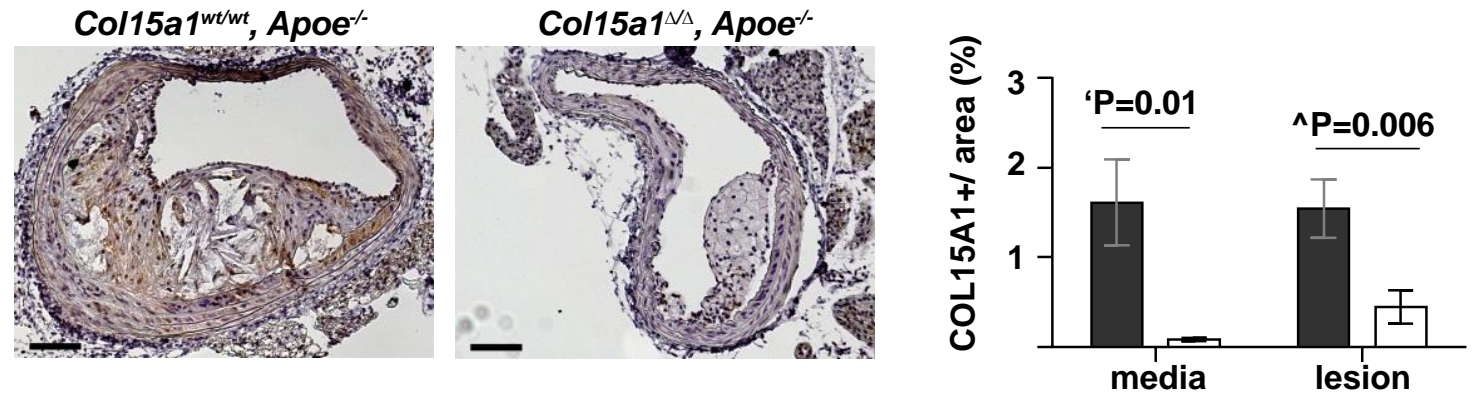

SMC YFP ${ }^{+/+}$Col15a1wtwt, Apoe ${ }^{-/}$ SMC YFP ${ }^{+/}$Col15a1 $^{\Delta / \Delta}$, Apoe $^{-/-}$ 
Figure 2: Validation of specificity of SMC Col15a1 knockout, SMC lineage tracing mice.

(A) Excision of Col15a1 occurs only in presence of tamoxifen and shows nearly complete recombination in SMC. (B) SMC Col15a1 knockout is highly specific to SMC-rich vessels (aorta and carotid). (C, D) Staining for COL15A1 in advanced lesions from 18 week Western diet fed SMC YFP ${ }^{+/}$Col15a1 $^{\text {wtwt }}$, Apoe $^{-/}(\mathrm{n}=11)$ and SMC $\mathrm{YFP}^{+/+} \mathrm{Col}_{15 a 1^{\Delta /}}, \mathrm{Apoe}^{-/}(\mathrm{n}=13)$ mice show significant reduction of COL15A1 in both the media and lesion of SMC YFP ${ }^{+/+} \mathrm{Col15a1}^{\Delta / \Delta}, \mathrm{Apoe}^{-/-}$mice. Scale bars represent $100 \mu \mathrm{m}$. ' $P$ value was determined by unpaired, two-tailed $t$ test with Welch's correction. ${ }^{\wedge} P$ value was determined by Mann Whitney $U$ test. Values represent mean \pm s.e.m. 
Figure 3
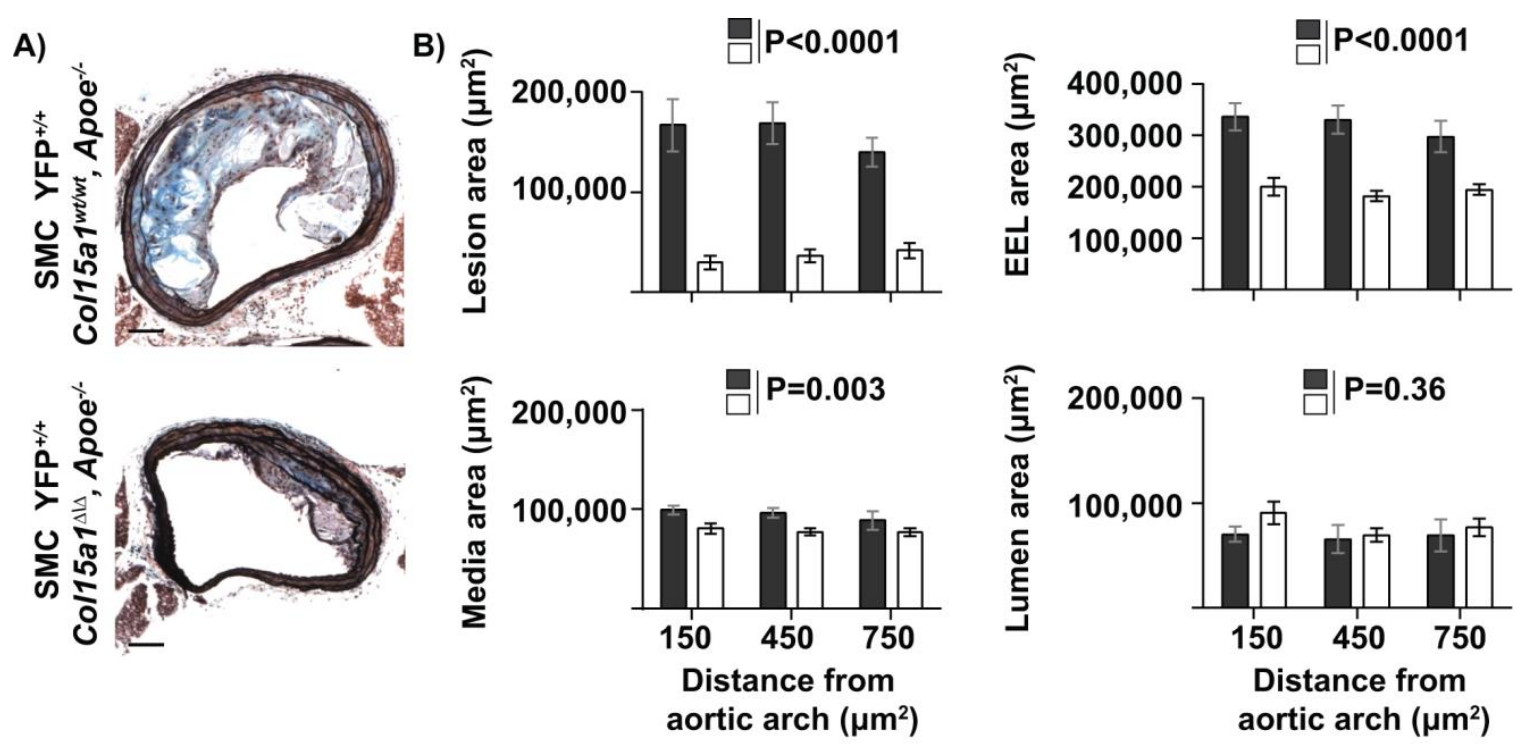

c)

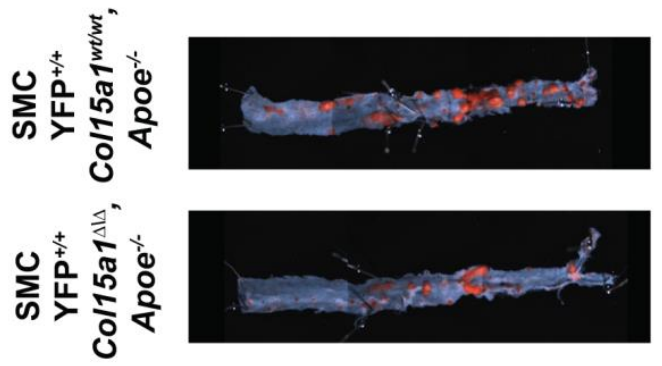

D)

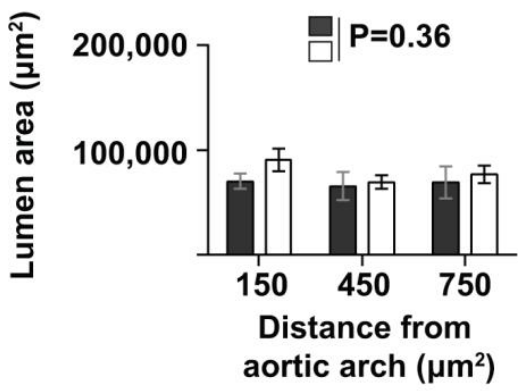

SMC YFP $^{+/+}$Col15a1wtwt, Apoe ${ }^{-/-}$

$\mathrm{SMC} \mathrm{YFP}^{+/+} \mathrm{Col15a}^{\Delta / \Delta}, \mathrm{Apoe}^{-/}$ 
Figure 3: Loss of SMC-derived COL15A1 resulted in a marked decrease in lesion size.

(A) Representative images of the BCA cross-sections from SMC $\mathrm{YFP}^{+/+}$ Col15a1 ${ }^{\text {wtwt }}$, Apoe $^{-/-}(\mathrm{n}=11)$ and SMC YFP ${ }^{+/+}$Col15a1 $^{\Delta / \Delta}$, Apoe $^{-/}(\mathrm{n}=13)$ mice fed a Western diet for 18 weeks stained with Movat reagent for lesion morphometry. Scale bars represent $100 \mu$ m. (B) Quantification of lesion area, external elastic lamina (EEL) area, media area, and lumen area measurements from Movat staining of the BCA. $P$ value was determined by two-way ANOVA, repeated measures. Representative images (C) and quantification (D) of Sudan IV stained aortas from SMC YFP ${ }^{+/+} C_{0} 15 a 1^{\text {wt/wt }}, \operatorname{Apoe}^{-/-}(\mathrm{n}=9)$ and SMC YFP $\mathrm{YF}^{+/+}$ Col15a ${ }^{\Delta \Delta}, A p o e^{-/-}(n=6)$ mice. ${ }^{\wedge} P$ value was determined by Mann Whitney $U$ test. Values represent mean \pm s.e.m. 
Figure 4

A) $\mathrm{SMC} \mathrm{YFP}+/+\quad S M C ~ \mathrm{YFP}^{+/+} \quad$ B) Col15a1 ${ }^{\text {wtwt }}$, Apoe ${ }^{-/}$

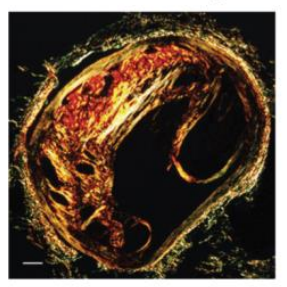

D) $\mathrm{SMC} \mathrm{YFP}^{+/+}$ Col15a1 ${ }^{\text {wtwt }}$, Apoe ${ }^{-/}$
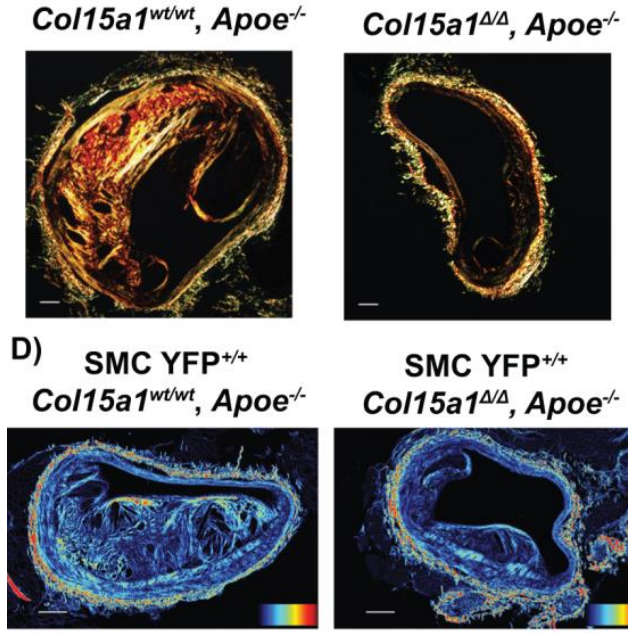

SMC YFP ${ }^{+/+}$

$\mathrm{Col}^{15 a 1^{\Delta / 4}}, \mathrm{Apoe}^{-/}$

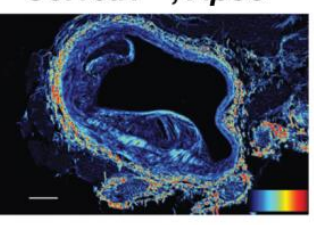

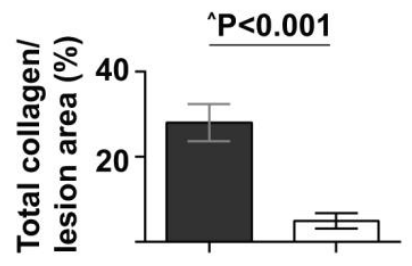

E)

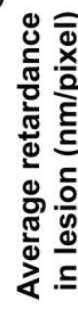

C)

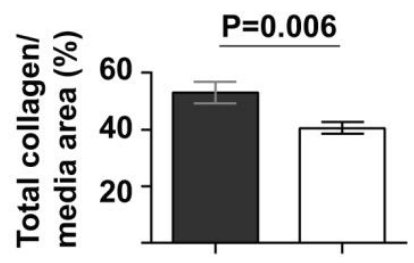

F)
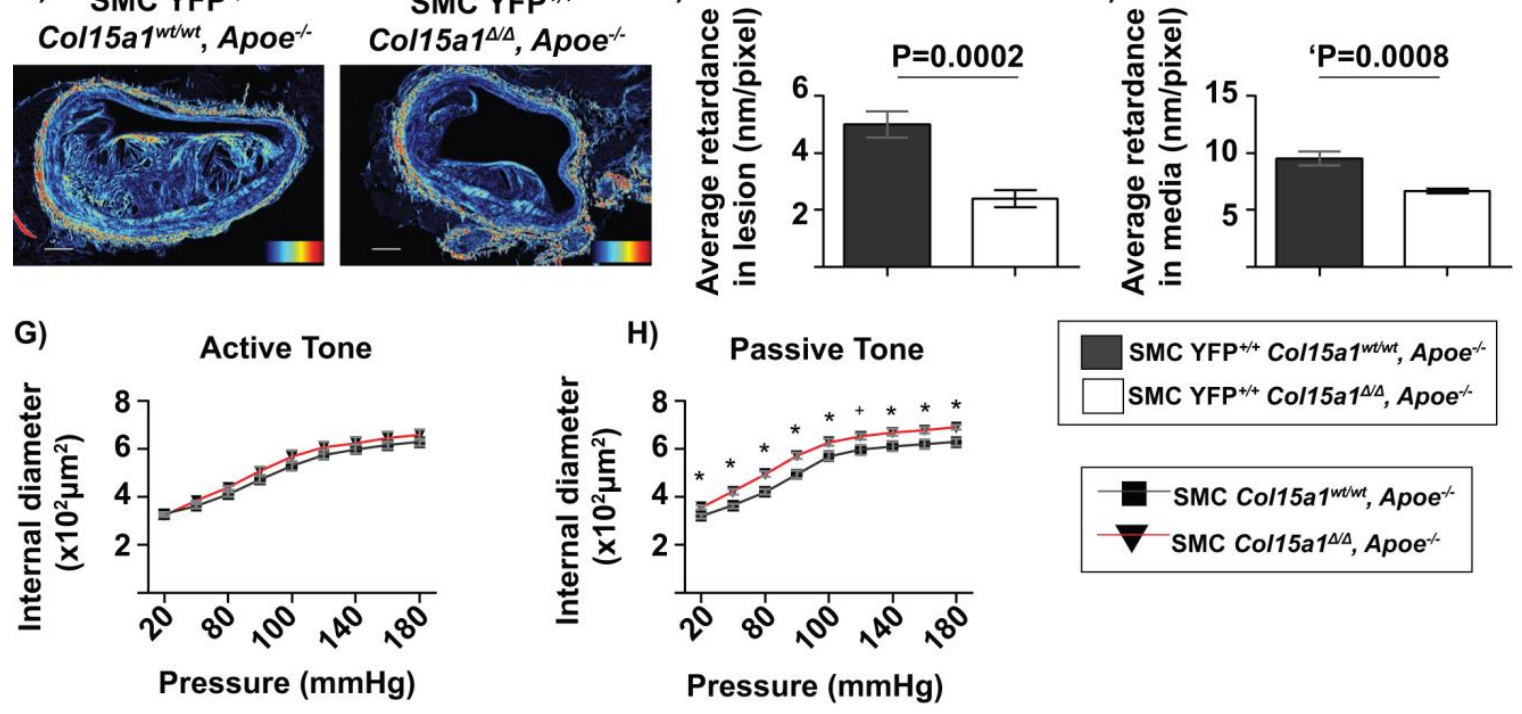

SMC YFP ${ }^{+/+}$Col15a1wtwt, Apoe SMC YFP ${ }^{+/+} \mathrm{Col15a1}^{\Delta / \Delta}$, Apoe ${ }^{-/-}$

- SMC Col15a1wtiwt, Apoe SMC Col15a1 ${ }^{\Delta / \Delta}$, Apoe $^{-/}$

Pressure (mmHg) 
Figure 4: Genetic inactivation of Col15a1 in SMC reduced collagen fiber content and maturation, and vessel elasticity.

(A) Representative images of the Picrosirius Red stained BCA under polarized

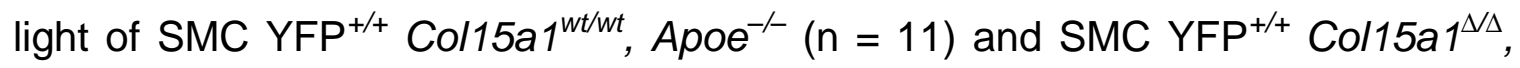
Apoe $^{-/}(\mathrm{n}=13)$ mice. Scale bars represent $100 \mu \mathrm{m}$. Quantification of total collagen content per lesion (B) and media (C) area. (D) Representative images of collagen maturation by LC-Polscope analysis where pixel intensity is proportional to birefringent, organized fibrillar collagen. Average retardance measurements indicate a decrease in lesion (E) and media $(F)$ collagen organization with SMC Col15a1 knockout. Heat map represents the amplitude of retardance $(\mathrm{nm})$ from 0 (black) to 39.5 (red). Scale bars represent $100 \mu \mathrm{M}$. $P$ value was determined by unpaired, two-tailed $t$-test. ' $P$ value was determined by unpaired, two-tailed $t$-test with Welch's correction. ${ }^{\wedge} P$ value was determined by Mann Whitney $U$ test. (G \& H) Quantification of pressure myography to assess the active and passive tone of carotid arteries of 18 week Western diet fed SMC Col15a1 ${ }^{\text {wtwt }}$, Apoe $^{--}(\mathrm{n}=6)$ and SMC Col15a ${ }^{\Delta / \Delta}, A_{p o e^{-/}}(n=5)$ mice. (G) No significant differences were detected at any point in active tone. $(\mathrm{H})$ Passive tone was significantly increased in SMC Col15a1 ${ }^{\Delta \Delta}$, Apoe $e^{-/}$compared to SMC Col15a $1^{\text {wtwt }}, \mathrm{Apoe}^{-/}$animals. For, passive tone, $P$ value was determined by unpaired, two-tailed $t$-test at each pressure. ${ }^{*} P$ value $<0.05$ and ${ }^{+} P$ value $<0.054$. Values represent mean \pm s.e.m. 
Figure 5

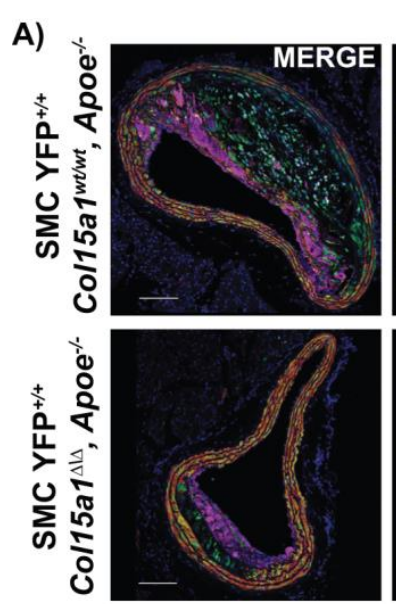

B)

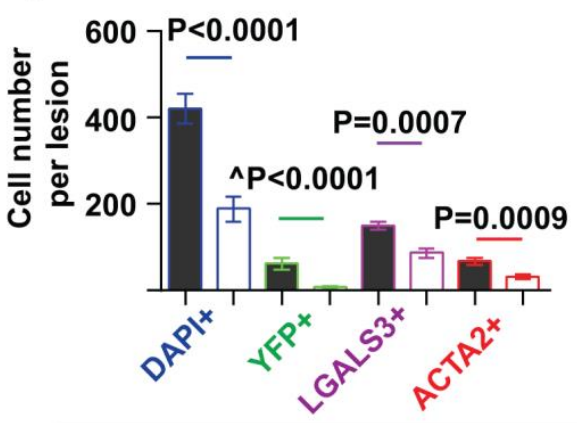

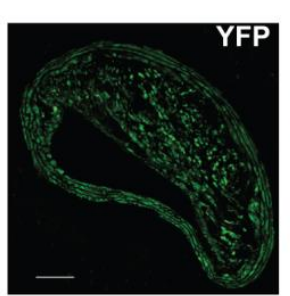

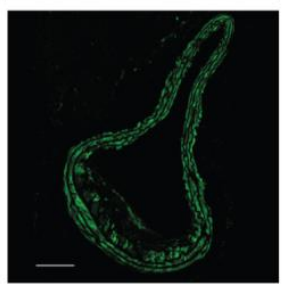

C)
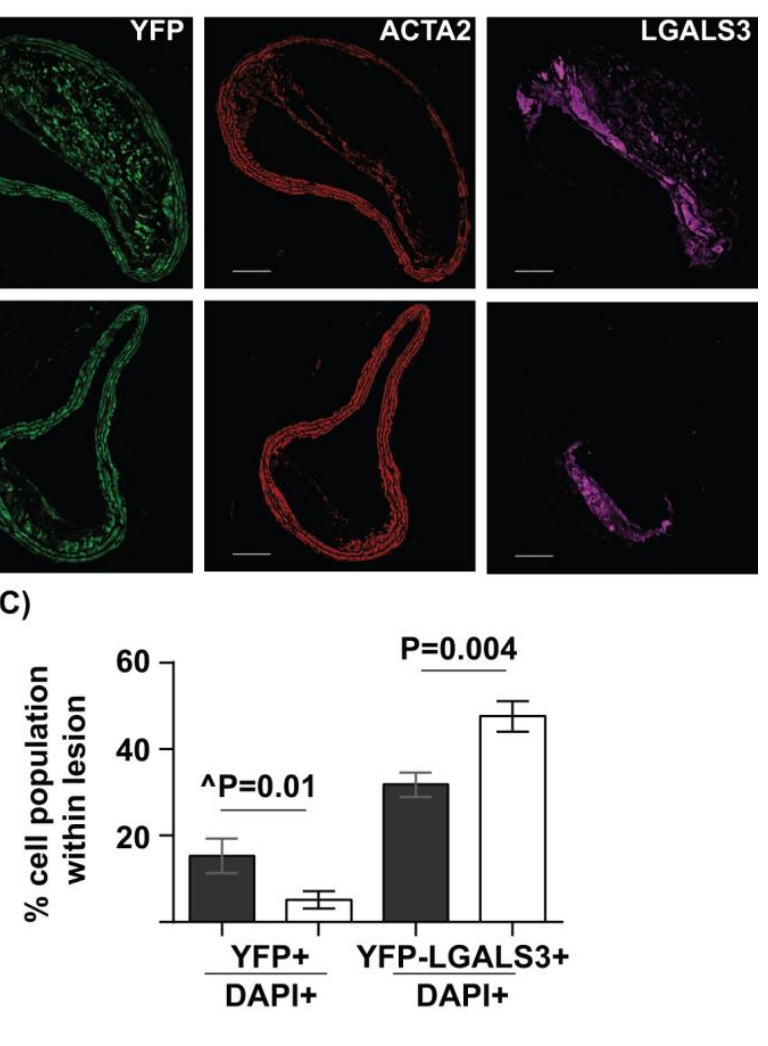
Figure 5: Loss of SMC specific COL15A1 resulted in overall reductions in the number of $\mathrm{YFP}^{+}$SMC-derived cells within the lesions but an increase in YFP $^{-}$LGALS3 $^{+}$macrophage cells.

(A) Representative immunofluorescence images of BCA sections of SMC YFP ${ }^{+/+}$ Col15a1 ${ }^{\text {wtwt }}$, Apoe $^{-/}(\mathrm{n}=11)$ and SMC YFP ${ }^{++}$Col15a1 $^{\Delta / \Delta}, A p o e^{-/}(\mathrm{n}=13)$ mice showing a marked decrease in overall cell number, a decrease in $\mathrm{YFP}^{+} / \mathrm{DAPI}^{+}$ cells and increase in $\mathrm{YFP}^{-} \mathrm{LGALS3}^{+} / \mathrm{DAPI}^{+}$cells within the lesions of the SMC $\mathrm{YFP}^{+/+} \mathrm{Col}_{15 a 1^{\Delta \Delta}}, \mathrm{Apoe}^{-/}$mice. Scale bars represent $100 \mu \mathrm{m}$. (B) Quantification of cell number per lesion stained positive for DAPI, YFP, LGALS3, or ACTA2 averaged across four locations of the BCA. (C) Quantification of percentage of SMC (YFP) and non-SMC-derived macrophages (YFP-LGALS3 ${ }^{+}$) over total number of cells $\left(\mathrm{DAPI}^{+}\right)$within lesions averaged across four separate locations of the BCA. (D) Quantification of cell density of the lesion by looking at cell number normalized to lesion area $\left(\mu \mathrm{m}^{2}\right)$. Given that SMC YFP ${ }^{+/} \mathrm{Col15a1}^{\Delta \Delta}, \mathrm{Apoe}^{-/}$have more acellular, necrotic core regions in their lesions as compared to that of SMC $\mathrm{YFP}^{+/+}$Col15a ${ }^{\Delta / \Delta}, A p o e^{-/}$mice, we also (E) quantified cell density of the lesion by looking at cell number normalized to the lesion area $\left(\mu \mathrm{m}^{2}\right)$ with the necrotic core regions excluded. $P$ value was determined by unpaired, two tailed $t$-test. ${ }^{\wedge} P$ value was determined by Mann Whitney $U$ test. Values represent mean \pm s.e.m. 
Figure 6

A)
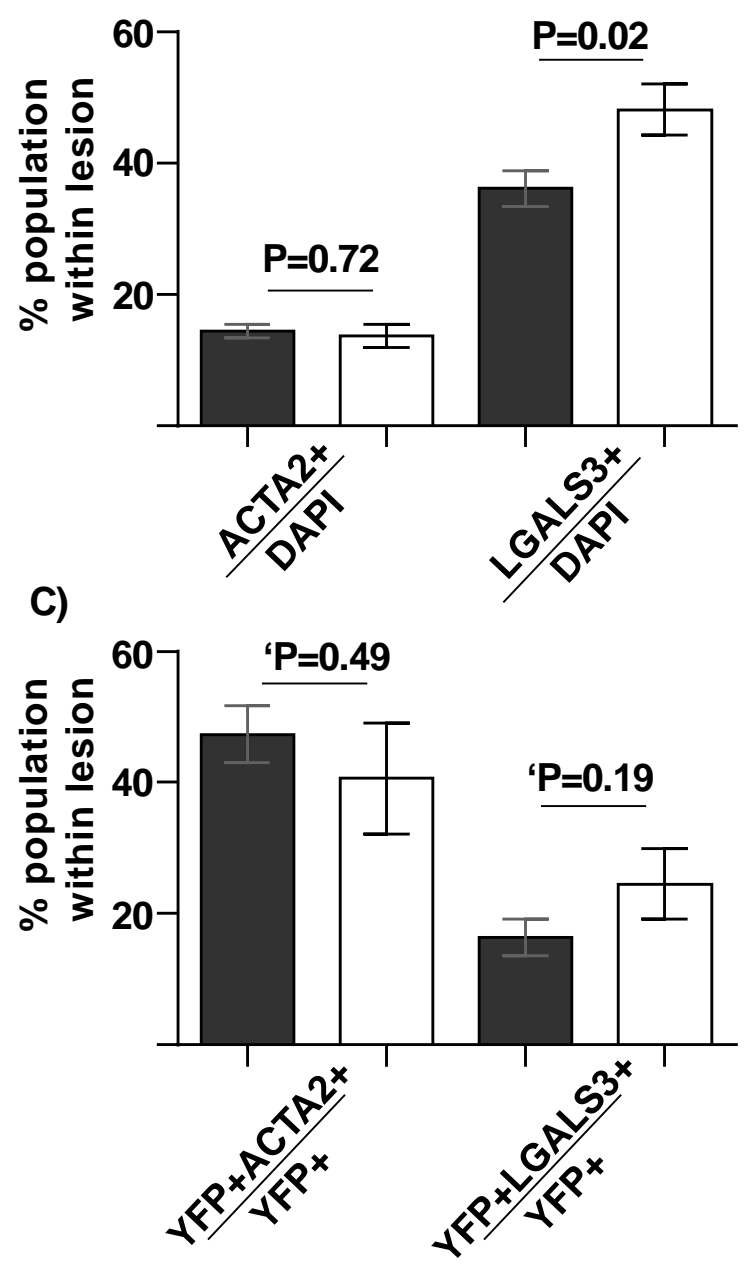

B)

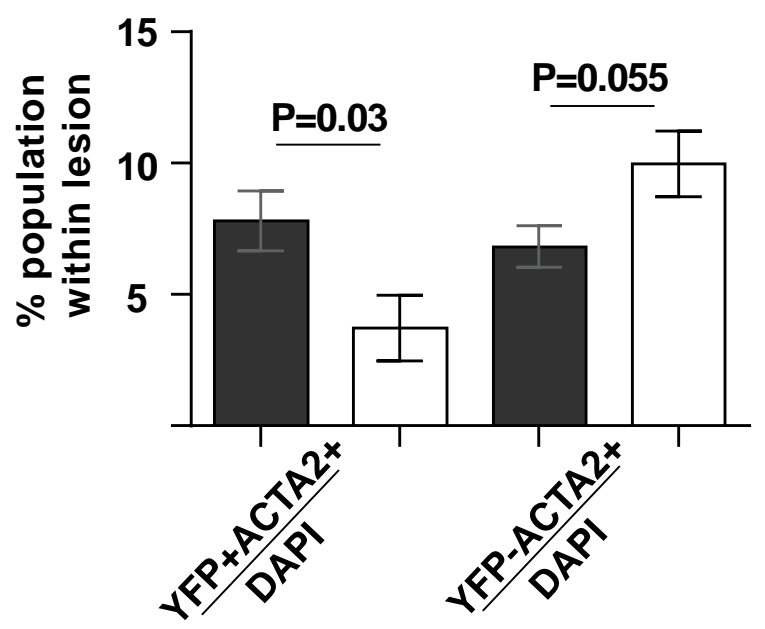

SMC YFP ${ }^{+/+}$Col15a $^{\text {wtwt }}$, Apoe ${ }^{-/}$ $\square$ SMC YFP $^{+/+}$Col15a1 $^{\Delta / \Delta}$, Apoe $^{-/}$ 
Figure 6: SMC-derived cell populations were reduced with SMC Col15a1 knockout.

(A) No changes were observed in percentage of $\mathrm{ACTA}^{+} / \mathrm{DAPI}{ }^{+}$cells but a significant increase was found in the percentage LGALS3 ${ }^{+} / \mathrm{DAPI}^{+}$cells in SMC $\mathrm{YFP}^{+/+}$Col15a1 $^{\mathrm{\Delta \Delta}}$, Apoe $^{-/}(\mathrm{n}=13)$ compared to SMC $\mathrm{YFP}^{+/+}$Col15a1 ${ }^{\mathrm{wt} w t}, \mathrm{Apoe}^{-/-}$ $(n=11)$ lesions. (B) A significant decrease in SMC-derived ACTA2 $^{+}$ $\left(\mathrm{YFP}^{+} \mathrm{ACTA}^{+} / \mathrm{DAPI}^{+}\right)$cells and a near significant increase in non SMC-derived $\mathrm{ACTA2}^{+}\left(\mathrm{YFP}^{-} \mathrm{ACTA2}^{+} / \mathrm{DAPI}^{+}\right)$cell populations were also found in SMC Col15a1 knockout compared to wild type. (C) No significant differences were seen in the proportion of SMCs able to express ACTA2 $\left(\mathrm{YFP}^{+} \mathrm{ACTA} 2^{+} / \mathrm{YFP}^{+}\right)$or LGALS3 $\left(\mathrm{YFP}^{+} \mathrm{LGALS3}^{+} / \mathrm{YFP}^{+}\right)$as a consequence of SMC Col15a1 knockout. Values represent the average of three locations across the BCA for each genotype. $P$ value was determined by unpaired two-tailed $t$-test analysis. ' $P$ value was determined by unpaired two-tailed $t$-test analysis with Welch's correction. Values represent mean \pm s.e.m. 
Figure 7

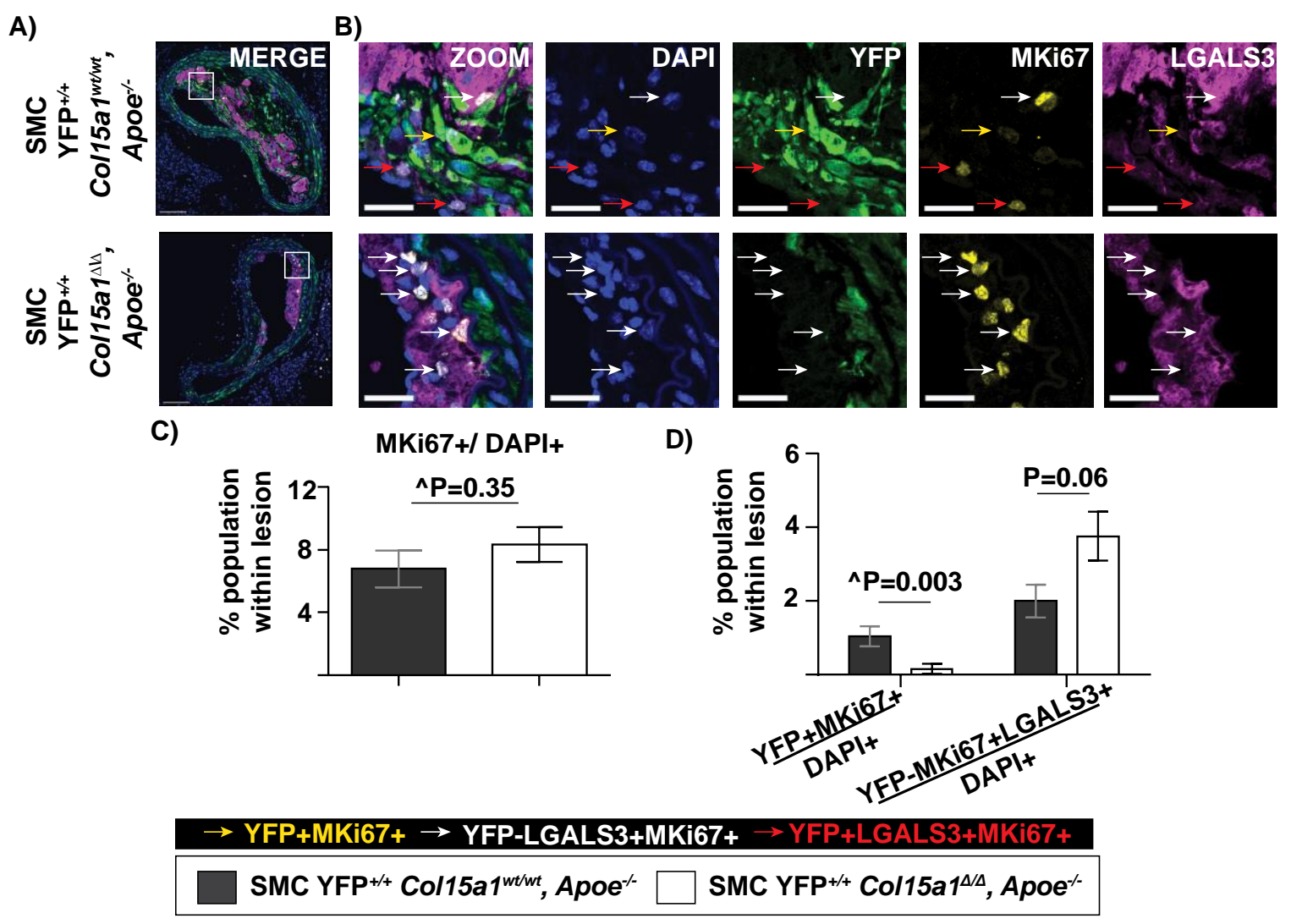


Figure 7: SMC specific Col15a1 knockout was associated with a decrease in lesion SMC proliferation.

(A) Representative immunofluorescence images of BCA sections stained for proliferating cells $\left(\mathrm{MKi67}^{+}\right)$within SMC $\mathrm{YFP}^{+/+}$Col15a1 $^{\text {wtwt }}$, Apoe $^{-/}(\mathrm{n}=11)$ and SMC $\mathrm{YFP}^{+/+} \mathrm{Col}_{15 a 1^{\Delta \Delta}}$, Apoe $^{-/}(\mathrm{n}=13)$ lesions. (B) Higher magnification and single marker immunostaining of white box region in (A). Arrows indicate subpopulations of proliferating cells including SMC (YFP ${ }^{+} \mathrm{MKi} 67^{+}$, yellow arrows), non SMC-derived macrophages (YFP ${ }^{-}$GALS3 $^{+}$MKi67 ${ }^{+}$, white arrows), and SMCderived macrophage-like cells (YFP ${ }^{+}$LGALS3 ${ }^{+} M K i 67^{+}$, red arrows). Scale bars represent $100 \mu \mathrm{m}(\mathrm{A})$ and $25 \mu \mathrm{m}(\mathrm{B})$. (B) Quantification of the percentage of proliferating cells within the lesion $\left(\mathrm{MKi} 7^{+} / \mathrm{DAPI}^{+}\right)$showed no difference between

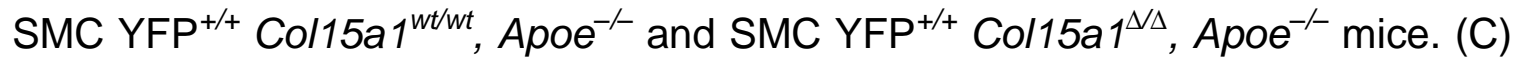
However, there was a significant decrease in the percentage of SMC-derived cells $\left(\mathrm{YFP}^{+} \mathrm{MiKi67} 7^{+} / \mathrm{DAPI}^{+}\right)$and an increase in the percentage of non SMCderived macrophages $\left(\mathrm{YFP}^{-} \mathrm{MKi} 67^{+} \mathrm{LGALS}^{+} / \mathrm{DAPI}^{+}\right)$proliferating within SMC

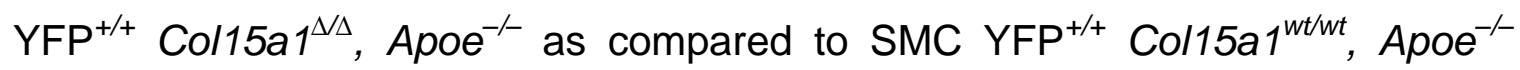
lesions. $P$ value was determined by unpaired, two-tailed $t$-test. ${ }^{\wedge} P$ value was determined by Mann Whitney $U$ test analysis. Values represent the mean \pm s.e.m of one location of the BCA. 
Figure 8

A)
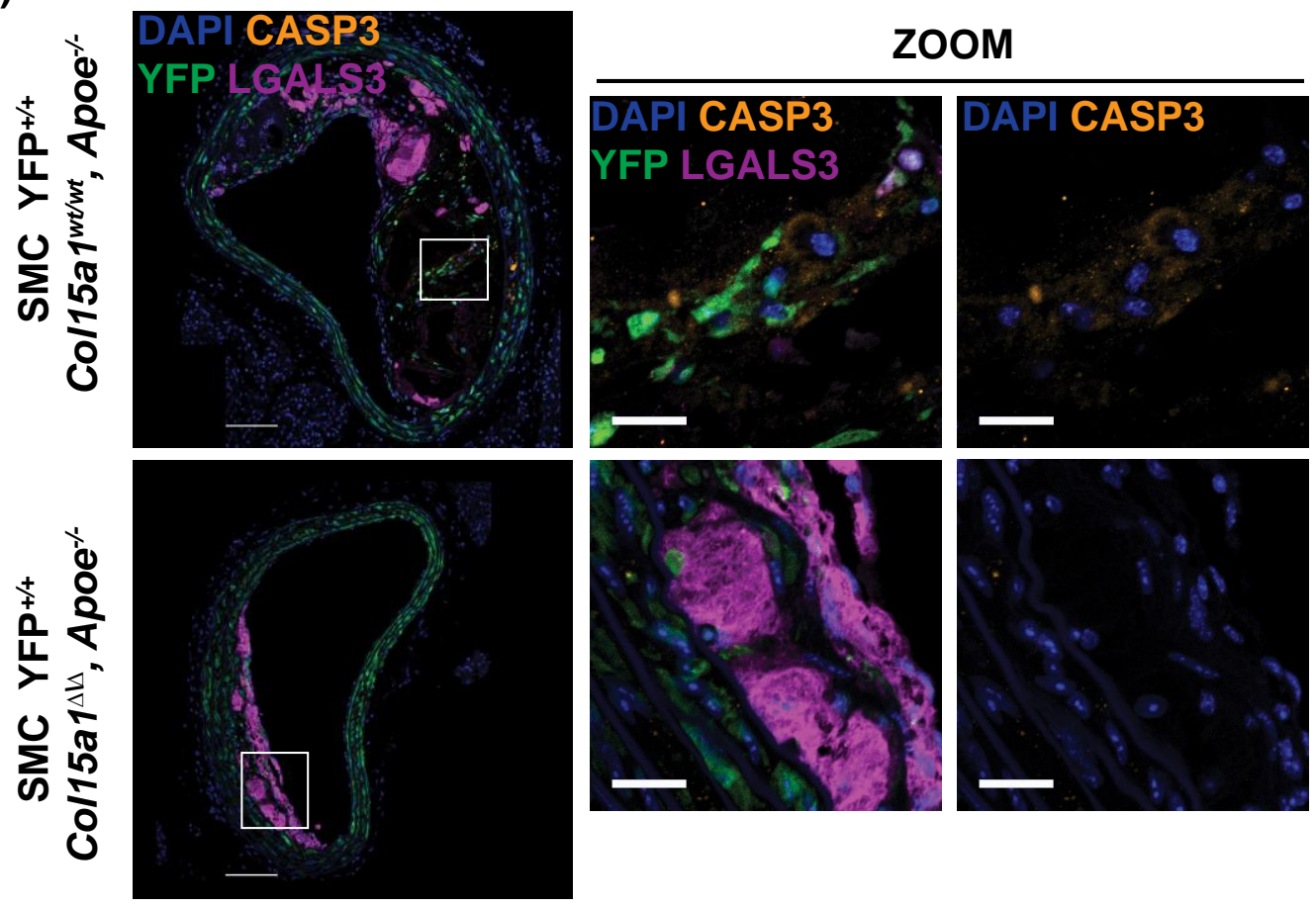

B)

CASP3+

C) CASP3+/ DAPI+
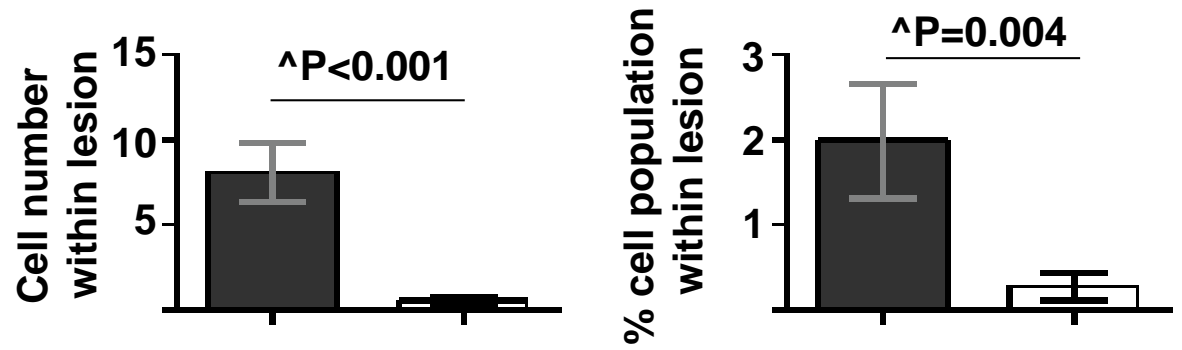

SMC YFP ${ }^{+/+}$Col15a $1^{\text {wtwt }}$, Apoe $^{-/-}$

SMC YFP ${ }^{+/+}$Col15a1 $^{\Delta / \Delta}$, Apoe $^{-/-}$ 
Figure 8: SMC Col15a1 knockout resulted in a decrease in lesion cell death. (A) Representative immunofluorescent images of $\mathrm{SMC} \mathrm{YFP}^{+/+}$Col15a1 ${ }^{\text {wtwt }}$, Apoe $^{-/}(\mathrm{n}=11)$ and SMC YFP ${ }^{+/}$Col15a1 $^{\Delta \Delta}$, Apoe $^{-/}(\mathrm{n}=13)$ lesions stained for cell death marker cleaved caspase $3\left(\mathrm{CASP}^{+}\right)$. (B) Quantification of CASP3 ${ }^{+}$ cells counted per lesion and (C) percentages of $\mathrm{CASP}^{+}$cells within lesions $\left(\mathrm{CASP}^{+} / \mathrm{DAPI}^{+}\right)$show SMC $\mathrm{YFP}^{+/+} \mathrm{Col}_{15 a 1^{1 / \Delta}} \mathrm{Apoe}^{-/}$mice have significantly

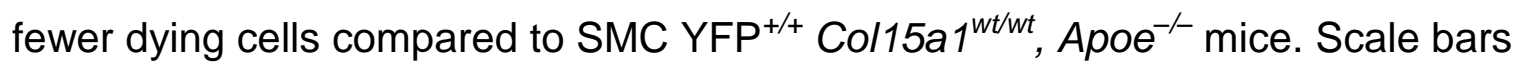
represent $100 \mu \mathrm{m}$ and on zoom in images $25 \mu \mathrm{m}$. ${ }^{\wedge} P$ value from Mann Whitney $U$ test. Values represent mean \pm s.e.m. 


\section{Figure 9}

A)

Ingenuity Pathway Analysis of Pathways Significantly Dysregulated with SMC Col15a1 ${ }^{1 \Delta}$

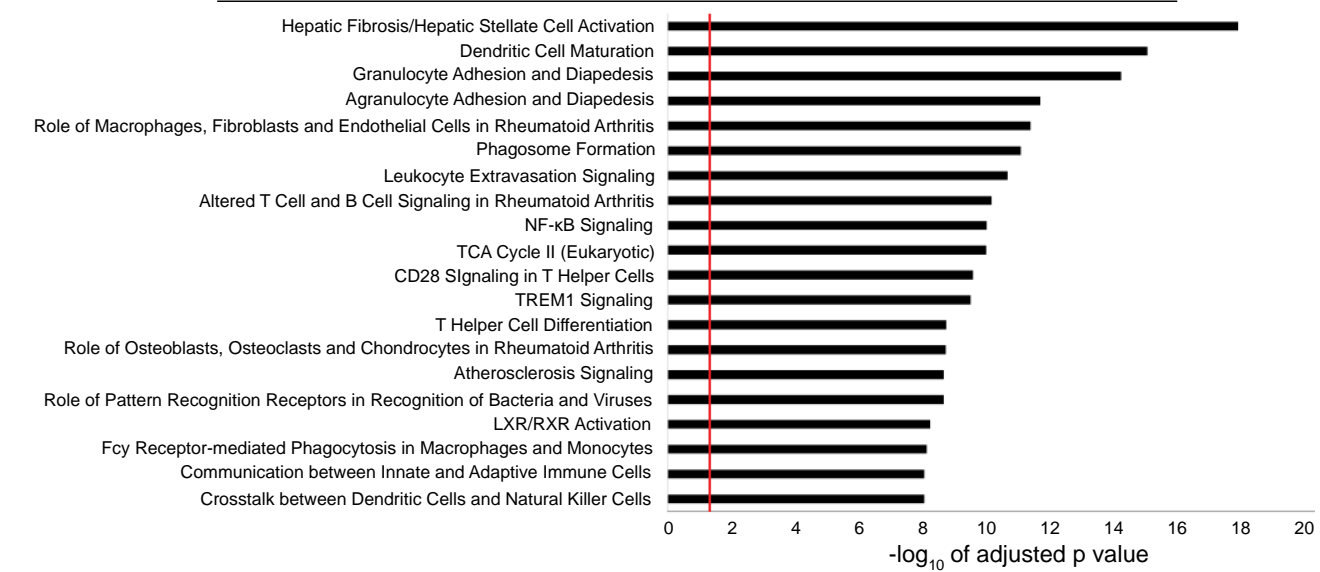

B)

Upstream Regulator Analysis

Lipopolysaccharide

Tumor Necrosis Factor (TNF)

Transforming Growth Factor Beta 1 (TGFB1)

Interferon gamma (IFNG)

Colony stimulating factor 2 (CSF2)

Rosiglitazone

Dexamethasone

Interleukin 6 (IL6)

Tretinoin

Interleukin 1 beta (IL1B)

Phorbol myristate acetate

Erb-B2 Receptor Tyrosine Kinase 2 (ERBB2)

Genistein

Peroxisome proliferator-activated receptor gamma (PPARG)

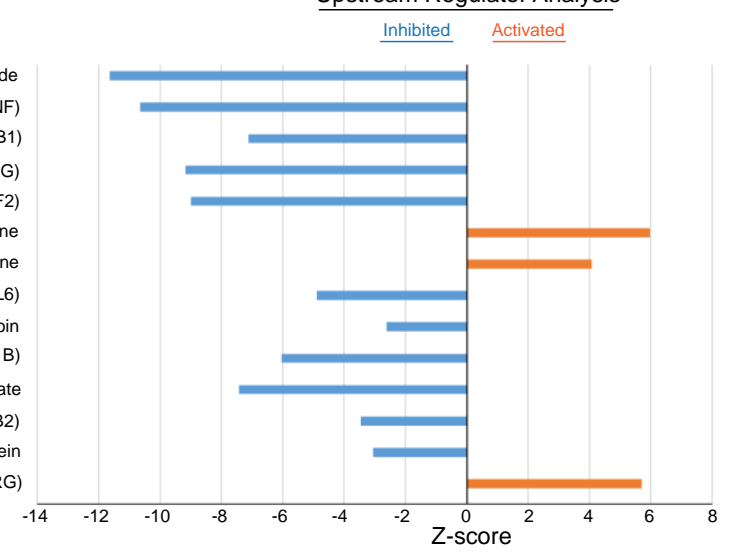

$\underline{P \text { value }}$

6.07E-97

3.24E-79

$1.77 \mathrm{E}-68$

$5.01 \mathrm{E}-67$

$7.78 \mathrm{E}-58$

$1.00 \mathrm{E}-54$

1.57E-54

3.15E-50

2.35E-46

1.67E-43

$6.82 \mathrm{E}-43$

4.15E-42

5.63E-38

$6.19 \mathrm{E}-37$ 
Figure 9: SMC specific Col15a1 knockout resulted in downregulation of immune cell processes that impact atherosclerotic plaque formation.

RNA-seq analysis of the BCA, aortic arch, and carotid artery specimens from 18 week Western diet fed SMC YFP ${ }^{+/+}$Col15a1 $^{\Delta / \Delta}$, Apoe $^{-/}(\mathrm{n}=3)$ compared to SMC $\mathrm{YFP}^{+/+}$Col15a1 ${ }^{\mathrm{wt} w t}, \mathrm{Apoe}^{-/-}(\mathrm{n}=3)$ mice. (A) Ingenuity Pathway Analysis showed dysregulation of inflammatory pathways. Red line marks cut off for significantly enriched (adjusted $P$ value $\leq 0.05$ ) pathways. Enrichment is shown as $-\log _{10}$ of adjusted $P$ value. (B) Upstream Regulator Analysis was performed on the top approximately 3,000 differentially expressed genes containing a log fold change of \pm 0.30 from the RNA-seq to identify master regulators that are either inhibited or activated as a consequence of SMC Col15a1 knockout. 
Figure 10

A)

COL15A1
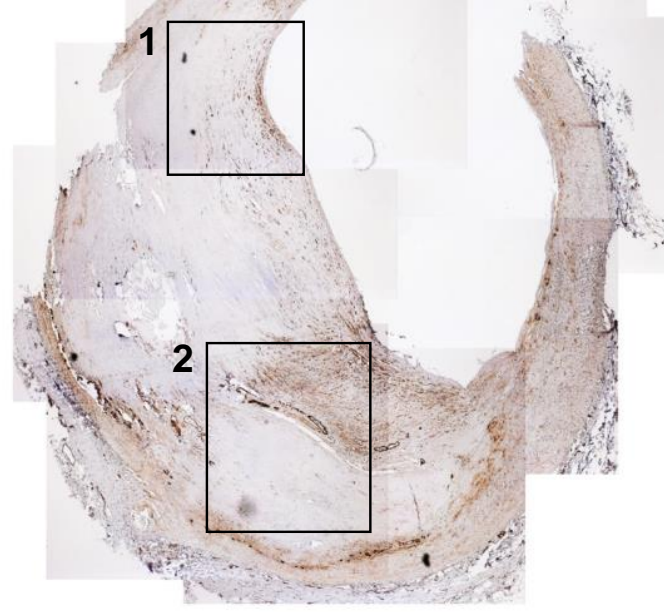

C)

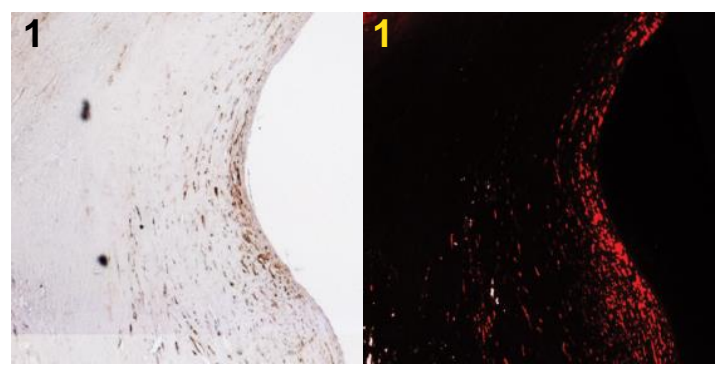

B)

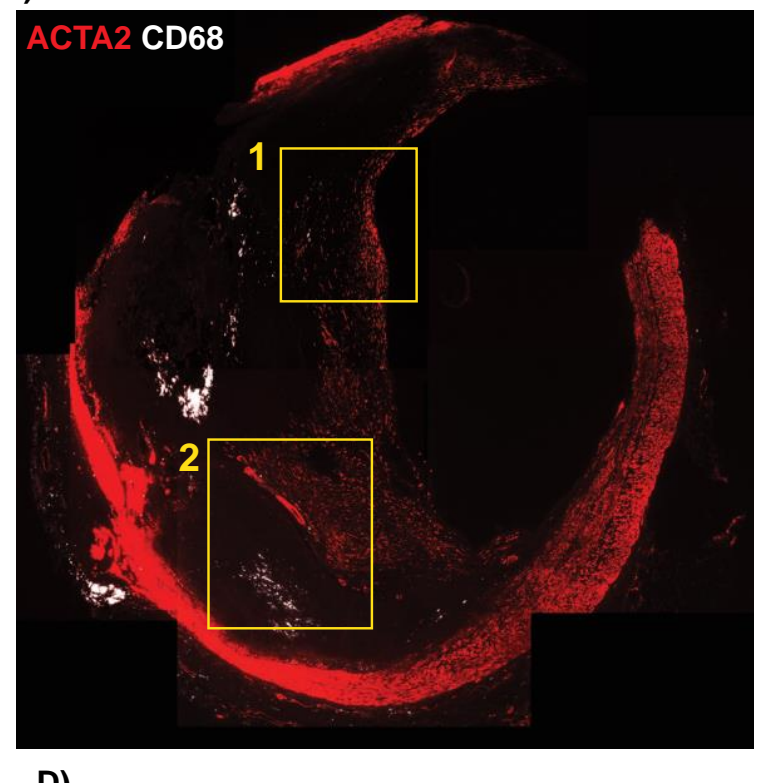

D)

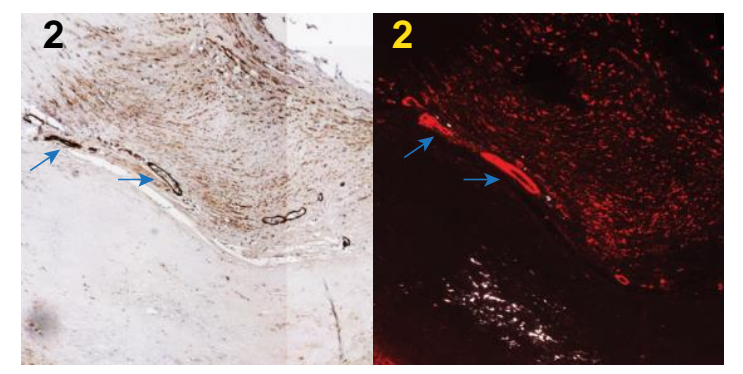


Figure 10: COL15A1 is pervasive within advanced human atherosclerotic plaques, and localized to the fibrous cap and intraplaque microvessels.

A representative image of a human coronary artery lesion from one of six patients analyzed who underwent coronary artery bypass surgery. Serial sections were stained with $(\mathrm{A}) \mathrm{COL15A} 1$ or $(\mathrm{B})$ traditional macrophage (CD68) and SMC marker genes (ACTA2). (C, D) Boxes $(1,2)$ represent regions of higher magnification to highlight COL15A1 presence in the ACTA2 rich fibrous cap (C, D) and intraplaque microvessels ( $D$, blue arrows) with little to no COL15A1 staining in CD68 rich regions (D). 
Table 1: No differences in cholesterol, triglyceride or body weight between SMC $\mathrm{YFP}^{+/+} \mathrm{Col}_{15 a 1^{\mathrm{wt} / \mathrm{wt}}, \mathrm{Apoe}^{-/} \text {and SMC YFP }}{ }^{+/+} \mathrm{Col}_{15 a 1^{\Delta \Delta}}, \mathrm{Apoe}^{-/-}$mice.

\begin{tabular}{|c|c|c|c|}
\hline & $\begin{array}{c}\text { SMC YFP }^{+/+} \\
\text {Col15a1 } 1^{\mathrm{wt} w t}, \text { Apoe }^{-/} \\
(\mathrm{n}=20)\end{array}$ & $\begin{array}{c}\text { SMC YFP }^{+/+} \\
\text {Col15a1 }^{\Delta \Delta \Delta}, \text { Apoe }^{-/} \\
(\mathrm{n}=20)\end{array}$ & $\begin{array}{c}\mathrm{P} \\
\text { value }\end{array}$ \\
\hline Cholesterol, $\mathrm{mg} / \mathrm{dL}$ & $1147 \pm 79.49$ & $973.6 \pm 74.93$ & 0.12 \\
\hline Triglycerides, $\mathrm{mg} / \mathrm{dL}$ & $152.9 \pm 12.40$ & $148.6 \pm 15.83$ & $0.34^{\wedge}$ \\
\hline Body weight, $\mathrm{g}$ & $35.77 \pm 0.86$ & $34.04 \pm 1.20$ & $0.11^{\wedge}$ \\
\hline
\end{tabular}

$P$ value is unpaired two-tailed $t$-test, $\wedge^{\wedge} P$ value $=$ Mann Whitney $U$ test; mean \pm s.e.m.

Table 2: Genotyping and excision primers for Col15a1.

\begin{tabular}{|c|c|}
\hline \multicolumn{2}{|c|}{ Col15a1 (TransViragen \& BGD) } \\
\hline DOC346 & GTGCCTCGGTCCCTTAAAAGCC \\
\hline BGD15ex1R & АCCCGCGCTCTGAAACCCGACT \\
\hline DOC349 & GGGATAGTTTGGCAGTGTGTTGTCAC \\
\hline $\begin{array}{l}\text { Doc346-BGD15ex1R- } \\
\text { Doc349: }\end{array}$ & $\begin{array}{l}\text { Col15a1 }{ }^{\text {wt/wt }}: 754 \mathrm{bp} \\
\text { Col15a1 }^{\text {fl/tI }:} 872 \mathrm{bp} \\
\text { Col15a1 }^{\Delta / \Delta}: 339 \mathrm{bp}\end{array}$ \\
\hline
\end{tabular}


CHAPTER 3

Conclusions and Future Directions 


\section{Conclusions}

The goal of this thesis was to determine if SMC-derived COL15A1 influences the development of advanced atherosclerotic lesions. The results presented in Chapter 2 show that loss of SMC produced COL15A1 led to a marked attenuation in atherosclerotic lesion development. In stark contrast to the advanced atherosclerotic lesions seen in wild type animals, SMC Col15a1 knockout animals developed small macrophage-rich and SMC and collagen-poor lesions that were fatty streak like in appearance despite 18 weeks of Western diet feeding. Taken together, the data in Chapter 2 of this dissertation present the first definitive evidence that a collagen derived solely from SMC can influence atherosclerotic lesion development with loss of SMC produced COL15A1 drastically impairing advanced lesion formation.

\section{Discussion and Future Directions}

In this section, I further elaborate on some of the key mechanisms I uncovered in the studies presented in Chapter 2. In addition, I address remaining unanswered questions from these studies and propose future experiments in order to guide the next steps in the study of COL15A1 and COL15A1 in atherosclerosis. 
2.1. SMC produced COL15A1 is important in the formation and organization of fibrillar collagens within the vessel wall and in atherosclerotic lesions.

COL15A1 is important for the organization of fibrillar collagens in atherosclerotic lesion development. In our SMC Col15a1 knockout animals, we observed a reduction in collagen content and maturation within the media of 18 week Western diet fed mice (Figure 4A-F). Furthermore, SMC Col15a1 knockout animal carotid arteries had an increased passive tone indicative of a less stiff vessel (Figure 4G-H). Moreover, we observed a marked reduction in collagen content and organization within the lesions of SMC Col15a1 knockout animals as compared to wild type controls (Figure 4A-F). Combined, these data provide evidence that SMC-derived COL15A1 is important for maintenance of vessel wall fibrillar collagen organization, content, and functional stability which stands in agreement with previously published studies ${ }^{23,25,26}$.

A critical unanswered question in our studies, however, is exactly how loss of SMC-derived COL15A1 influences collagen and extracellular matrix formation and organization. Based on my studies outlined in Chapter 2 and those conducted previously by others ${ }^{23,25,104,118,153}$, one possibility is that COL15A1 physically binds to fibrillar collagens and actively remodels the fibrillar collagen network. To definitively determine if this occurs in vivo would require new technologies that have the ability to do live cell imaging of fibrillar collagen matrix remodeling in the media and lesions of SMC Col15a1 knockout and wild type mice throughout atherosclerotic lesion development. An indirect in vivo approach to confirm that COL15A1 leads to changes in collagen matrix structure would be 
to perform electron microscopy and quantify the porosity, dispersion, and fiber alignment angle of the fibrillar collagen network in the media and lesion of 18 week Western diet fed SMC Col15a1 knockout and wild type mice. While this does not directly test COL15A1 action, it would provide further correlative evidence and insight into the net effect of loss of SMC produced COL15A1 on collagen and extracellular matrix architecture. An in vitro collagen gel contraction or remodeling assay to test whether SMC produced COL15A1 can bind and remodel a fibrillar collagen matrix is also a viable option. This experiment would involve plating Col15a1 knockout and wild type SMC on fluorescently labelled fibrillar collagen type I coated plate ${ }^{154}$ or in a three-dimensional fibrillar collagen lattice ${ }^{96}$, treating cells with atherogenic factors (e.g. oxidized phospholipids), and quantifying changes in fibrillar collagen type I porosity, dispersion, and fiber alignment.

Another consideration is that knockout of Col15a1 in SMC reduces SMC specific production of other collagens, specifically fibrillar collagens. One way to test this would be to isolate SMC from the media of the BCA, carotids, and aorta of 18 week Western diet fed SMC Col15a1 knockout and wild type animals via flow cytometry and perform RNA-seq to determine if collagen expression is reduced in Col15a1 knockout SMC. An in vitro option would be to treat Col15a1 knockout and wild type SMC with a combination of atherogenic growth factors, cytokines, and oxidized phospholipids and screen for changes in RNA and protein expression in all of the nearly 30 collagen types. The limitations of these approaches are that: 1) flow sorting of aortic SMC may change the transcriptional 
profile of the SMC; and 2) the in vitro experiments may not fully recapitulate the atherosclerotic lesion environmental milieu.

Lastly, the $71 \%$ reduction in lesion COL $15 A 1$ staining we observed in our SMC Col15a1 knockout mice allows us to conclude that SMC are the major producer of COL15A1 within lesions (Figure $2 \mathrm{C}$ ). It is intriguing to speculate that SMC are also overall a major source of collagen in atherosclerotic lesions in vivo. Testing this hypothesis directly in vivo, however, would require generation of inducible SMC specific knockouts for each of the nearly 30 collagen types and subtypes plus long-term Western diet feeding.

\subsection{SMC produced COL15A1 impacts various pro-inflammatory pathways and nearly all immune cell types.}

The results of the in vivo RNA-seq presented in this dissertation indicate that the cumulative effect of loss of SMC-derived COL15A1 is the inhibition of various pro-inflammatory pathways including IFNY, TNFa, TGF $\beta$, and IL1 $\beta$ (Figure 9). In addition, SMC Col15a1 knockout lesions displayed an overall reduction in the number of LGALS3 $^{+}$cells within lesions but also showed an increase in the proportion of non-SMC-derived macrophages per total lesion cells $\left(\mathrm{YFP}^{-} \mathrm{LGALS3}^{+} / \mathrm{DAPI}^{+}\right.$, Figure $\left.5 \mathrm{C}\right)$ and a nearly significant increase in proliferative capacity of non-SMC-derived macrophages as compared to wild type lesions $\left(\mathrm{YFP}^{-} \mathrm{MKi67}^{+} \mathrm{LGALS3}^{+} / \mathrm{DAPI}^{+}\right.$, Figure 7D). Moreover, we know from my studies in Chapter 2 and previous studies by other groups ${ }^{23-26}$ that COL15A1 
is important in fibrillar collagen organization, and is produced and influential in atherosclerotic lesion development.

One possible explanation for the phenotype we observe with SMC Col15a1 knockout is loss of SMC produced COL15A1 induces changes in the extracellular matrix organization within the media impairs immune cell recruitment and adhesion that initiates atherosclerosis ${ }^{8,41,111,127,155}$. Like cultured SMC, cultured monocytes and macrophages also change their function in response to collagen state ${ }^{13}$. For example, plating human monocyte derived macrophages on monomeric fibrillar collagen type I enhanced cell adhesion, spreading, and differentiation while plating on polymerized fibrillar collagen type I blocked cell adhesion and spreading ${ }^{13,156,157}$. To assess this in vivo, we could perform live cell intravital multiphoton microscopy as used and developed Klaus Ley at the La Jolla Institute ${ }^{158}$ to track monocyte interactions with atheroprone areas of the carotid arteries of our SMC Col15a1 knockout and wild type animals during early initiation of atherosclerosis (i.e. 6-8 weeks of Western Diet feeding). This method requires fluorescent labeling of myeloid derived cells to detect monocyte interactions and movement. We would therefore need to cross our SMC lineage tracing, SMC specific Col15a1 knockout and wild type animals with a separate not Cre-driven myeloid cell lineage tracing system (e.g. Drerecombinase targeting rox sites) to create a dual SMC and myeloid cell lineage tracing mouse model system. Another option would be to transplant fluorescently labeled bone marrow (e.g. tdTomato) into lethally irradiated SMC Col15a1 
knockout and wild type animals prior to placing the animals on diet and then perform intravital multiphoton microscopy imaging.

An in vitro approach to determine whether the collagen matrix produced by Col15a1 knockout SMC impairs adhesion of myeloid cells would be to have SMC generate a matrix with and without COL15A1. I attempted to do this by treating SMC Col15a1 knockout and wild type SMC with 10\% serum containing media to promote secretion and formation of an extracellular matrix in culture. Once the matrix is formed, SMC can be removed through calcium chelation and subsequent hypotonic shock. Macrophages (i.e. bone marrow derived macrophages or peritoneal macrophages) could then be plated on top of the matrices, and time to and number of macrophages adhering quantified. Early attempts at optimizing generation of a SMC Col15a1 knockout matrix from SMC in vitro were promising (Figure 11). However, success was inconsistent. In preliminary experiments where SMC were plated back on their matrix, it was discovered that SMC preferentially bound to regions without matrix. The SMC generated matrix also deteriorated within 48 hours of removal of SMC. If pursued, much more optimization will need to be done. It also may be advantageous to use the Col15a1 knockout and wild type SMC to generate a three-dimensional matrix to perform these macrophage adhesion experiments.

A separate possibility for the small, under-developed lesion we observe may be that SMC Col15a1 knockout results in reduction of SMC specific production of pro-inflammatory and macrophage recruiting factors. To test this, we could perform a cytokine array on media from cultured Col15a1 knockout and 
wild type SMC treated with oxidized lipids which can initiate atherogenesis. An alternative method would be to take ex-vivo aortic media explants from Western diet fed SMC Col15a1 knockout and wild type animals, place them in cell culture media for 24-48 hours, and perform a cytokine array on the cell culture media to determine if SMC Col15a1 knockout leads to reduced production of proinflammatory and immune cell factors. Ideally, the cytokine array would contain factors we identified as SMC-COL15A1 dependent in our in vivo RNA-seq Upstream Regulator Analysis as well as other cytokines and growth factors implicated in atherosclerotic lesion development. As a complement to these studies, staining can be done for cytokine array candidates in normal and early atherosclerotic vessels of SMC Col15a1 knockout and wild type mice.

Lastly, it is possible that the global reduction in pro-inflammatory pathways we observe is simply a reflection of the SMC Col15a1 knockout and wild type lesions being at different stages in lesion development. Our SMC Col15a1 knockout lesions exhibit the characteristics of early stage atherosclerotic lesions in that they are small and fatty streak like in that lesions contain a paucity of SMC and collagen, are proportionally enriched in macrophages, and are nearly devoid of a fibrous cap. This stands in stark contrast to the advanced stage lesions seen in the wild type animals. The impairment in lesion development in SMC Col15a1 knockout animals despite 18 week Western diet feeding suggests that important COL15A1-mediated changes are likely occurring during the initiation or early development of atherosclerotic lesions. Future studies looking at early stages in lesion initiation and progression therefore may be crucial to our understanding of 
how SMC produced COL15A1 may be inhibiting pro-inflammatory pathways and immune cell function in lesion development.

\subsection{COL15A1 as a modulator of cell proliferation.}

COL15A1 appears to be an important modulator of cell proliferation and influences proliferation in a cell-specific manner. In our studies, we found that SMC Col15a1 knockout led to a significant decrease in overall number of SMC and proliferating SMC within lesions and a near significant increase in non-SMCderived macrophage proliferation (Figure $7 \mathrm{C}$ ). With respect to SMC, our prior in vitro data showed that siRNA mediated Col15a1 knockdown decreased human aortic SMC proliferative capacity ${ }^{21}$. Of interest, work by Feil et $a^{20}$ and Chappell et al. ${ }^{60}$ have used two separate SMC specific lineage tracing model, a SM22aCreER $^{\mathrm{T} 2}$ model and a Myh11-CreER ${ }^{\mathrm{T} 2} /$ Confetti model respectively, to provide convincing in vivo evidence that SMC undergo oligoclonal expansion as a means of populating lesions ${ }^{60}$. Notably, we initially identified Col15a1 expression as upregulated in an in vitro human aortic SMC model of replicative senescence or oligoclonal expansion ${ }^{21}$. It is reasonable therefore to speculate one plausible explanation for the reduction in SMC and SMC proliferation in SMC Col15a1 knockout versus wild type lesions may be through SMC-COL15A1 mediated inhibition of SMC clonal expansion.

This concept is further supported by work in the field on COL15A1 in cancer models that observed associations between COL15A1 and cancer cell proliferation and clonal expansion. Amenta et al. used histological staining of 
COL15A1 in normal, pre-metastatic, and metastatic breast ductal carcinoma tissue to conclude that reduced levels of COL15A1 in the ductal basement membrane preceded metastasis ${ }^{159}$. Separately, overexpression of COL15A1 in D98 AP2 human cervical carcinoma cells that were then injected into mice showed dose-dependent suppression of tumor formation ${ }^{13,121}$. However, overexpression of COL15A1 in cultured D98 AP2 cells and BxPC-3 human pancreatic epithelial carcinoma cells showed no differences in proliferation ${ }^{106,113,121}$. Taken together with our own observations of SMC Col15a1 knockout in atherosclerosis, it is therefore possible that the cellular sources and/or spatial locations where COL15A1 is secreted are critical. For example, in our model, loss of SMC-derived COL15A1 may have proportionally different effects on particular cells (e.g. SMC versus macrophages) and at different locations (i.e. media SMC vs lesion SMC). With respect to the near significant increase in macrophage proliferation observed in our SMC Col15a1 knockout, it is highly probable that changes in their proliferation are due to secondary effects as a consequence of reduced SMC COL15A1 production.

Another question remains, however, as to whether the reduced SMC proliferative capacity within SMC Col15a1 knockout lesions is the result of direct or indirect consequences of reduced SMC Col15a1 transcription and/or reduced overall COL15A1 levels in the lesions. One way to answer this question would be to isolate aortic SMC from SMC Col15a1 knockout and wild type mice and determine the population doubling rates of the SMC in culture. If, as expected, you see a decrease in SMC proliferation in Col15a1 knockout SMC, the next step 
would be to transfect SMC with plasmids that overexpress full length COL15A1 used previously by others in the field ${ }^{106,113,121}$ to see if the proliferative deficit that occurs with SMC Col15a1 knockout is ameliorated. A concern in generating primary SMC lines from the aortas of SMC Col15a1 knockout mice is that the cultured cells will have a selective pressure bias toward proliferation as proliferation is necessary for cell survival in culture. An alternative approach therefore would be to use wild type SMC lines and perform siRNA-mediated Col15a1 knockdown. An issue with this knockdown approach is that COL15A1 will likely still be present and secreted from the cells albeit at a lower level that that of the siRNA non-targeted control treated SMC. To fully block COL15A1 production by SMC, these siRNA experiments should be done in conjunction with agents that either: 1) block secretion of COL15A1; or 2) sequester COL15A1 (i.e. neutralizing antibody or binding to COL15A1 trimerization domain) to prevent its potential signaling functions and incorporation into the extracellular matrix.

\subsection{Is there an alternative cell source of COL15A1 within lesions?}

In our SMC Col15a1 knockout lesions, we observed a $71 \%$ decrease in lesion COL15A1 content as compared to wild type lesions (Figure 2C \& D). Given we have a $95 \%$ reduction in COL15A1 in the SMC-rich media of SMC Col15a1 knockout mice indicative of high efficiency of Col15a1 knockout in SMC, the remaining COL15A1 in SMC Col15a1 knockout lesions is likely produced by another cell type. Atherosclerotic lesions contain a wide variety of cell types including SMC, macrophages, endothelial cells, B cells, T cells, fibroblasts, 
among others ${ }^{41,160-163}$. Cultured macrophages and endothelial cells, however, have been shown to have the capacity to produce collagens and other extracellular matrix components. Specifically, work by Jürgen Rauterberg's group has shown that cultured primary human peripheral blood monocytes and macrophages, and the THP-1 macrophage cell line express nearly all collagen types with all three expressing COL15A1 in culture ${ }^{164,165}$. Separately, human umbilical vein endothelial cells (HUVEC) have been show to produce collagen IV, laminin and fibronectin under hypoxic ${ }^{166}$ and extended culture conditions ${ }^{167}$.

However, there are two major limitations in definitively determining which cell types are secreting collagens in atherosclerotic lesions. First, studies by our lab ${ }^{14-16}$ and others ${ }^{17,18,20,58,60}$ have recently provided convincing evidence that marker gene expression which has been classically used to identify SMC, macrophages, and endothelial cells, are not cell-specific within atherosclerotic lesions. For example, macrophages and endothelial cells can express SMC markers (e.g. ACTA2 and TAGLN respectively) and vice versa thereby precluding definitive determination of these cell types without the use of rigorous lineage tracing models ${ }^{15-20}$. To that point, we also need to carefully re-evaluate conclusions drawn from previous studies in which lineage tracing was not used and cell types were identified solely by marker gene expression. Second, collagens are secreted proteins therefore co-localization of collagens with a cell marker does not indicate if that cell actually produces collagens. Consequently, a large unknown in the field existed as to which cells actually produce collagens within atherosclerotic lesions. The studies in this dissertation in which we use a 
SMC specific genetic knockout of Col15a1 therefore represents the first evidence that SMC are a major source of a collagen, COL15A1, in atherosclerosis and that COL15A1 produced solely by SMC can severely impact atherosclerotic lesion development.

One way to circumvent these limitations and test what other cell type is producing COL15A1 within lesions would be to do fluorescence in situ hybridization (FISH) for Col15a1 mRNA in advanced mouse lesions from cellspecific lineage tracing mice. Current studies in the Owens lab by Alexandra Newman looking at atherogenesis in SMC lineage tracing mice (Myh11-CreER ${ }^{T^{2}}$ ROSA26-STOP floxed eYFP $A p o e^{--}$) that have been lethally irradiated and received a bone marrow transplantation with fluorescently labeled tdTomato bone marrow and by Olga Cherepanova looking at atherogenesis in endothelial cell-specific lineage tracing mice (Cdh5-CreER ${ }^{\text {T2 }}$ ROSA26-STOP floxed eYFP $A p o e^{-/}$) will yield myeloid and endothelial cell-specific lineage tracing advanced atherosclerotic lesion samples for the proposed Col15a1 mRNA FISH studies. FISH for Col15a1 mRNA in lesions from these animals will provide direct evidence of whether Col15a1 is expressed in myeloid derived and/or endothelial cells. However, a caveat of this experiment is we are making the assumption that a non-SMC source will produce COL15A1 even in the presence of SMC produced COL15A1. If that assumption is incorrect, we could: 1) generate a dual lineage tracing mouse model in which you have SMC specific lineage tracing, SMC Col15a1 knockout mice and endothelial cell-specific lineage tracing; or 2) perform a fluorescently labeled tdTomato bone marrow transplant into irradiated 
SMC Col15a1 knockout and wild type animals and feed both Western diet for 18 weeks. In both these models, it would still be necessary to then do FISH for Col15a1 mRNA in lesions to test if endothelial cells or myeloid cells express Col15a1.

An alternative method would be to use the novel in situ hybridizationproximity ligation assay (ISH-PLA) designed by Delphine Gomez, a former Owens lab member ${ }^{16}$. This method involves in situ hybridization of a biotinylated DNA probe to the Col15a1 promoter and proximity ligation assay between an antibody directed to biotin and an antibody for serine 5 phosphorylated RNA

polymerase II, a marker of gene activation. While arguably more technically difficult than FISH, I have successfully designed and performed ISH-PLA for serine 5 phosphorylated RNA-Pol II on the Col15a1 promoter in advanced mouse atherosclerotic lesions (Figure 12). Based on these results, subsequent follow up studies might include developing endothelial cell or myeloid cell specific lineage tracing, Col15a1 knockout animals to assess the role of myeloid or endothelial cell derived COL15A1 in atherosclerotic lesion development.

\subsection{Does loss of SMC COL15A1 production result in delayed and/or halted progression of atherosclerotic lesion development? Does COL15A1 affect all locations of the arterial tree similarly?}

In Chapter 2, we show that SMC Col15a1 knockout animals fed 18 weeks of Western diet exhibit a drastic $78 \%$ reduction in BCA lesion size and fail to form advanced lesions as observed in wild type controls. Additionally, SMC Col15a1 
knockout lesions have the characteristics of early fatty streak lesions including being SMC and collagen-poor, macrophage-rich, and are nearly devoid of an $\mathrm{ACTA}^{+}$fibrous cap. Based on these observations, an unanswered question remains as to whether SMC Col15a1 knockout animals have a delayed onset and/or halted progression of atherosclerotic lesion development. To answer this question, a future experiment would be to look at SMC Col15a1 knockout and wild type lesions at an earlier and later (i.e. 12 week 26 week Western diet feeding) time point and evaluate lesion development and indices of plaque stability.

In addition, we show in Chapter 2 that while SMC Col15a1 knockout results in significantly smaller BCA lesions as compared to wild type control lesions, there is no difference in Sudan $\mathrm{IV}^{+}$aortic lesion burden between the groups (Figure $3 C \& D$ ). Our lab typically focuses on the $B C A$ as the flow dynamics and lesions that develop in this region most closely mimic those of humans carotid artery lesions ${ }^{168}$. However, a large number of classic atherosclerotic lesion studies focus on the aortic root ${ }^{168}$. The aortic root, BCA and the thoracic and abdominal aorta are all derived from different embryonic origins $^{169}$ as well as are exposed to different flow dynamics ${ }^{168}$. Thus, there may be site specific atherosclerotic lesion development. A future direction would be to see if the changes in lesion size and characteristics observed in BCA are maintained in other regions such as in the aortic root of Chapter 2 SMC Col15a1 knockout and wild type mice. 
2.6. In our previous human studies, we found evidence that the human COL15A1 SNP is associated with decreased COL15A1 expression and increased age-related risk for atherosclerosis. In contrast, our mouse studies show loss of SMC produced COL15A1 results in attenuated lesion development. How can we rectify these observations? Moreover, does modifying COL15A1 content within lesions have therapeutic potential?

We initially showed in our previous manuscript on COL15A1 that there is a SNP within the human COL15A1 gene that conferred risk for atherosclerotic disease in individuals aged 55 and older ${ }^{21}$. In the human data in Figure 10 in Chapter 2, we provide the first description of where COL15A1 is expressed within advanced human coronary atherosclerotic lesions. Specifically, COL15A1 is highly expressed within the fibrous cap, plaque shoulders, and intraplaque microvessels which have all been implicated as important in plaque stability (Figure 10). Our conditional SMC specific lineage tracing, SMC specific Col15a1 knockout mice complement the human data by providing unambiguous evidence that SMC-derived COL15A1 is functionally critical for lesion development. That is, our mouse data show that loss of COL15A1 production exclusively in SMC in young 8 week old mice prior to the start of atherosclerosis leads to profound inhibition of lesion development (Figure 3), with lesions having an increased proportion of macrophages and decreased proportion of SMC per total lesion cells (Figure 5C), as well as a marked reduction in overall collagen content and maturation (Figures 4A-F). Together, these data provide the first detailed 
assessment of COL15A1 expression within mouse and human lesions and COL15A1 function in atherosclerotic lesion development.

Whether modifying COL15A1 levels are therapeutically efficacious has not yet been directly assessed due in part to the differences in experimental design and lineage tracing limitations in our mouse and human studies. The current clinical treatment for atherosclerosis is focused on interventional approaches to ameliorate risk factors ${ }^{40}$. Our genetic mouse model involved induction of loss of COL15A1 production by SMC in young mice that was then sustained throughout lesion development. This atherosclerosis prevention mouse study design and our findings therefore may not equate to the impact of atherosclerosis intervention studies which would involve modifying COL15A1 levels in already established late stage atherosclerotic lesions ${ }^{170}$. Experimental animal studies testing if reduction of COL15A1 levels in established advanced atherosclerotic lesions has beneficial effects on lesion composition and indices of plaque stability are therefore crucial to evaluate COL15A1 therapeutic potential. One potential future experiment design would be to use our mouse model but initiate knockout of COL15A1 production by SMC after 18 weeks of Western Diet, when advanced lesions have formed, and then assess indices of plaque stability and development after 26 weeks of Western diet feeding. Preliminary data in our lab using this experimental strategy has shown that this leads to labeling of $M y h 11^{+}$ cells within the fibrous cap region of the atherosclerotic plaque. The fibrous cap is a region where COL15A1 localizes and where fibrillar collagen organization is considered to be important for mechanical stability of lesions $8,13,43,79,102$. This 
study design would ideally lower COL15A1 levels but also allow us to assess if COL15A1 production by Myh11+ SMC within the fibrous cap of advanced lesions impacts atherosclerotic plaque stability. A caveat of this experiment is that we know from our data in Figures $2 \mathrm{C} \& \mathrm{D}$ that $\mathrm{COL} 15 \mathrm{~A} 1$ is present within 18 week Western diet lesions and can likely expressed by a non-SMC cell type. This may influence our experimental outcome and not lower COL15A1 lesion levels by much. Furthermore, it is important to note that a large portion of clinically used therapeutic agents are not cell specific. As such, an alternative experiment would be to feed inducible global Col15a1 heterozygous floxed mouse model

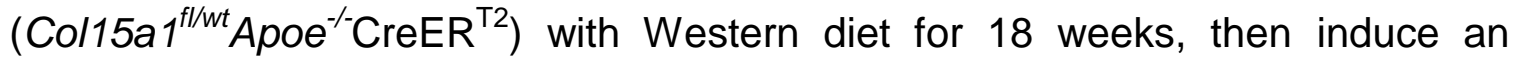
approximately $50 \%$ reduction in COL15A1 production by all cells, and assess lesion development and indices of stability after 26 weeks on Western diet. A limitation in both of these proposed study designs is we will be unable to identify the origin of cells within the lesions due to the absence of proper lineage tracing.

With respect to our human data, our previous studies of COL15A1 in human atherosclerosis provided correlative evidence that aged individuals harboring the risk allele have reduced COL15A1 expression and increased Sudan IV+ aortic lesion burden. ${ }^{21}$ However, only a small number of patients were sampled for this association and the analysis did not include measurement of indices of plaque stability. Future experiments in focusing on the association between COL15A1 levels, the COL15A1 SNP, and indices of plaque stability in patients will therefore be necessary to determine if COL15A1 has therapeutic and/or biomarker potential in humans. For example, a large scale study 
evaluating a potential correlation between COL15A1 levels within patient lesions and the incidence of S-T elevation myocardial infarctions (STEMI) or stroke would be ideal. In addition, we can also look at lesions from patients who have undergone coronary intravascular ultrasound (IVUS) and look at correlations between virtual histology measures of lesion size, calcification, and stenosis to determine if there is a correlation between these indices of plaque stability and the COL15A1 SNP in the patients' blood samples.

\subsection{What does COL15A1 directly interact with? Does it bind specific cell surface receptors?}

Currently, what COL15A1 directly binds to in vivo is not known. As such, a large amount of discovery based experiments will be needed to find COL15A1's binding partner in vivo. Immunogold staining for COL15A1 has shown by transmission electron microscopy (TEM) that COL15A1 binds to large collagen

fibers in the kidney, placenta, and colon ${ }^{23}$. However, the extracellular matrix fiber type COL15A1 is binding to (i.e. collagen type I, II, III etc.) in vivo is still a mystery. In the context of our studies, the most direct approach to discover what COL15A1 is binding to in vivo would be to immunoprecipitate COL15A1 from atherosclerotic aorta protein lysates and then run mass spectrometry. The next step would be to perform a co-immunoprecipitation for the candidate proteins found from mass spectrometry and COL15A1 in atherosclerotic aorta protein lysates to confirm binding. A limitation of this experiment is that we would not be able to determine the cell type involved in COL15A1 binding to the discovered 
protein using this method. A potential next step could be to then perform an in vivo proximity ligation assay (PLA) for COL15A1 and the candidate protein in atherosclerotic lesions in various cell-specific lineage tracing models to attain descriptive evidence that COL15A1 and the candidate protein are in close proximity to one another.

Separately, we could also take a SMC centric approach and search the literature for candidates from previous work on COL15A1 in vitro in non-SMC and see if these candidate genes are expressed by SMC and/or SMC in atherosclerosis. Work by Clementz et al. has shown that in cultured BxPC-3 pancreatic adenocarcinoma cells overexpressing full length human recombinant COL15A1, COL15A1 is in complex with discoidin domain receptor 1 (DDR1) and epithelial cadherin (E-cadherin) ${ }^{106}$. COL15A1 binding to DDR1 was also shown to inhibit DDR1 pathway signaling in these cells ${ }^{106}$. DDR1 is a collagen receptor expressed on SMC and macrophages that regulates SMC cell proliferation, migration and matrix remodeling ${ }^{85,171}$. E-cadherin is important for cell adhesion ${ }^{106}$. Interestingly, studies by Michelle Bendeck's lab in which DDR $1^{+/+}$ bone marrow was transplanted into DDR $1^{-/}$mice have shown an increase in aortic root atherosclerotic lesion size, cell number, collagen content, and fibrous cap area but decreased $\mathrm{ACTA}^{+}$and $\mathrm{MAC2}^{+}$cells within lesions ${ }^{85}$. Another observation is that multiplexin, an ortholog of both COL15A1 and COL18A1 present in Drosophila, binds to the Slit/Robo complex in Drosophila embryo Schneider S2 cells, co-localizes with Slit on the luminal surface of the cardiac tube during Drosophila embryonic development, and does not promote aorta 
development ${ }^{172}$. Interestingly, Slit/Robo complexes have been implicated in regulating cell migration during angiogenesis ${ }^{173}$. Lastly, solid-phase binding assays of COL15A1 purified from cultured human fibroblast and fibrocarcinoma cells has shown that purified COL15A1 binds to the collagen domain of fibronectin in vitro ${ }^{174}$. Fibronectin has been shown to promote SMC proliferation and signal through integrin $\mathrm{V}$ beta $3\left(\alpha_{\mathrm{V}} \beta_{3}\right)^{55,127}$.

To screen for what COL15A1 might be binding to in SMC, treatment of SMC with a mitogen present in atherosclerotic lesions to induce COL15A1 production will likely be needed as COL15A1 is expressed at low levels in cultured SMC at baseline. Platelet derived growth factor beta (PDGF-BB) may be an ideal choice of mitogen in this case as it is abundant in atherosclerotic lesions, acts through a single receptor (PDGFBR), induces COL15A1 production (data not shown), and promotes proliferation and migration of cultured $\mathrm{SMC}^{52}$. To screen for what COL15A1 might be binding to in SMC, we could start by assessing if siRNA knockdown of COL15A1 in SMC or Col15a1 knockout SMC in the absence or presence of PDGF-BB exhibit changes in protein expression of candidates DDR1, E-cadherin, SLITs, ROBOs, and/or fibronectin by Western blot. Based on these results, we could conduct the more challenging coimmunoprecipitation experiments for COL15A1 and the candidate protein(s) of interest in cultured cells and atherosclerotic aorta. Future work might also include a combination of gain and loss of function experiments for COL15A1 and the candidate protein(s) and quantifying SMC migratory and proliferative capacity. If none of the candidate proteins from the literature are shown to interact with 
COL15A1, a follow up experiment could be to run mass spectrometry on protein co-immunoprecipitated with COL15A1 from cultured SMC treated with and without PDGF-BB to determine the proteins COL15A1 is in complex with.

\subsection{The N-terminal domain and part of the collagenous domain of COL15A1} have glycosaminoglycan (GAG) binding sites containing mostly chondroitin sulfates. Could these GAG chains on COL15A1 be playing a role in the initiation of atherosclerotic lesion development?

COL15A1 contains GAG binding sites within its $\mathrm{N}$-terminal domain and at the start of its collagenous domain ${ }^{107}$. As COL15A1 GAG side chains consist mostly of chondroitin sulfates with some heparan sulfates, it has been labeled as a chondroitin sulfate proteoglycan ${ }^{22-24,104}$. A series of key studies conducted by Jan Borén's group discovered LDL binding proteins apoB100 and apoB48 bind to chondroitin sulfate proteoglycans in vitro ${ }^{146,146,175-177}$. The group went on to show that human apoB100, transgenic mice with a defective proteoglycan domain developed less atherosclerotic lesion burden ${ }^{175}$. Interestingly, these apoB transgenic mice exhibited a reduced atherosclerotic lesion burden as compared to control mice up until 20 weeks of atherogenic diet, at which point the apoB100 transgenic mice began to and ultimately did "catch up" to control mice disease burden by 40 weeks of atherogenic diet ${ }^{178}$. These studies contribute to the hypothesis that chondroitin sulfate containing proteoglycans are important in initial lipid retention in the media and initiate early lesion formation ${ }^{111,112}$. It is interesting to speculate that COL15A1, which is considered a chondroitin sulfate 
proteoglycan might contribute to atherosclerosis initiation by binding apoB100 or apoB48 and facilitate lipid retention within the media.

While an intriguing possibility, to the best of our knowledge there is currently no evidence that: 1 ) loss of SMC COL15A1 from medial SMC results in reduced lipid retention; or 2) that COL15A1 can bind apoB100, apoB48, or other lipoproteins. To answer the former, we could determine lipid retention in the media via Oil Red O staining of early atherosclerotic lesions in SMC Col15a1 knockout and wild type mice. Studies in SMC Col15a1 knockout and wild type mice fed 4 or 12 weeks Western diet, considered early time points in atherogenesis, have already been completed and are readily available for analysis. Oil Red O staining should be the first priority as it is the quickest way using existing reagents to testing our hypothesis. If SMC Col15a1 knockout animals do show reduced medial lipid retention at the earlier 4 and/or 12 week time points, it will be important to do follow up immunoprecipitation experiments to show in vivo that COL15A1 contains chondroitin sulfate modifications and that COL15A1 binds to apoB100 and/or apoB48 in atherosclerotic aorta. The immunoprecipitation experiments will likely need to be done at early atherosclerosis time points (e.g. at 10-13 weeks of Western diet feeding) as COL15A1 is nearly undetectable in large arteries at baseline (an observation held by myself and others ${ }^{25,117,147}$ ) but Col15a1 expression is upregulated in 13 week Western diet fed $A p o e^{-/}$mice ${ }^{21}$. To determine if COL15A1 in the media contains chondroitin sulfate modifications we will do the following: 1) scrape off lesions from vessels of 13 week Western diet aortae and immunoprecipitate 
COL15A1; 2) treat the immunoprecipitated COL15A1 with or without the enzyme chondroitinase; and 3) immunoblot for COL15A1 and look for a band shift to a smaller molecular weight with chondroitinase treatment indicative that COL15A1 has chondroitin sulfate proteoglycan side chains ${ }^{24,104}$. Lastly, to test the possibility that COL15A1 can bind to apoB100 or apo48 within the media, we will immunoprecipitate apoB100 or apoB48 from atherosclerotic aortas then immunoblot with COL15A1 and vice versa.

\subsection{What about COL15A1 C-terminal cleavage product restin? Could it be playing a role in atherosclerosis?}

As mentioned above, COL15A1 contains a C-terminal protein domain cleavage product restin ${ }^{22,107}$. Restin is highly expressed in the heart and skeletal muscle but is found in lower levels in the kidney, liver, and aorta ${ }^{147}$. As stated above, restin has $61 \%$ homology with endostatin, the COL18A1 C-terminal protein domain cleavage product ${ }^{115,147}$. The high homology between restin and endostatin led to the presumption in the field that the two had similar functions. Endostatin is a proven bioactive anti-angiogenic molecule shown to inhibit endothelial cell proliferation and migration ${ }^{147,179-181}$. In a seminal study by O'Reilly et al., recombinant endostatin drastically regressed primary Lewis lung carcinomas, T241 fibrosarcoma, EOMA hemangioma, and B16F10 melanoma tumors in mice ${ }^{180}$. Moreover, recombinant human endostatin is currently part of 67 clinical trials, most of which are cancer focused, in the United States (Endostatin search on clinicaltrials.gov). 
The few studies that have looked at restin, however, have not provided much clarity on a function for the molecule. Studies by Ramchandran et al. showed recombinant restin inhibits FGF induced cultured endothelial cell migration and attenuates growth of 786-O renal cell carcinoma tumor progression in mice though to a significantly lesser extent than recombinant endostatin ${ }^{115}$. In contrast, studies by Mutolo et al. showed that overexpression of restin in D98 AP2 human cervical carcinoma cells that were then injected into nude mice had no impact on tumor formation ${ }^{113,121}$. A definitive function for restin therefore remains largely ambiguous and unexplored. As such, an interesting future project will be to determine how/if restin is involved in atherogenesis. Unfortunately, use of commercial antibody mediated detection of restin in atherosclerotic aorta will likely be inconclusive as the antibody also labels full length COL15A1. To that point, Eklund et al. used an antibody for the C-terminal protein domain of COL15A1 that overlapped with the restin domain to prove loss of COL15A1 in their global Col15a1 knockout mice ${ }^{26}$. It is important to note that in all the studies in Chapter 2, an antibody to the N-terminal protein domain of COL15A1 was used for COL15A1 detection in order to ensure detection of only full length COL15A1. In order to determine if restin is present within atherosclerotic aortas, you can do high performance liquid chromatography, isolate the bands around 22 kilodaltons which has been identified as the size of restin, and then run on mass spectrometry to definitively identify restin. Depending on the results of these experiments, the next steps could be to develop an inducible transgenic 
overexpression restin mouse or a transgenic restin knockout mouse for use in a model of diet-induced atherosclerosis.

\subsection{Does COL15A1 have a role in the microvasculature?}

An unanswered question from our analysis is whether blood pressure is changed with SMC Col15a1 knockout in the microvasculature and if that may be a contributing factor to the reduced lesion size we observe in the BCA. Within the microvasculature of our SMC specific lineage tracing mouse model, we know that arteriole SMC and a subset of Myh11+ pericytes in capillaries are labeled with our YFP SMC lineage tracing tag upon tamoxifen injection (unpublished). Dysfunction in blood pressure and vessel stiffness in the microvasculature normally precedes development of atherosclerotic lesions ${ }^{182}$. Hypertension, a chronic increase in systemic blood pressure, is also an established risk factor for atherosclerotic disease development ${ }^{31,44,183-186}$. Notably, COL15A1 is also highly expressed in both arterioles and capillaries $25,26,117,187$. Studies in global Col15a1 knockout mice have no changes in blood pressure, however the heart, skin and skeletal muscle capillaries were kinked and disorganized; skin and skeletal muscle capillaries also showed increased vascular permeability in young adult mice $^{25}$. In our SMC lineage tracing, SMC Col15a1 knockout animals fed a Western diet for 18 weeks, we observed an increase in carotid artery passive tone indicative of reduced stiffness and a function for COL15A1 in large vessel tone (Figure $4 \mathrm{H})$. 
Despite these results, we did not measure blood pressure or study the microvasculature architecture in our SMC Col15a1 knockout animals. A crucial future study will be to do either tail cuff or carotid implantable telemetry measurements to determine if there is a reduction in baseline and/or Westerndiet fed mice blood pressure as a consequence of SMC Col15a1 knockout that might be a contributing factor to our phenotype. It will also be essential to look at the heart, skin, and skeletal muscle microvasculature as well as other microvasculature beds (e.g. retina, mesentery, and adipose tissue) to see if we observe a similar disorganized, ruptured microvasculature in our SMC Col15a1 knockout mice as was reported within the global Col15a1 knockout mice. Based on the results of these inquires, a prospective direction of future study might include looking at SMC Col15a1 knockout in pathological angiogenesis using a hind-limb ischemia or corneal burn model; both models are currently being used by Daniel Hess in the Owens Lab.

\subsection{Is there a connection between IL1 $\beta$ and COL15A1?}

We have made the following observations that suggest a connection between COL15A1 and IL1 $\beta$ and/or IL1R1 signaling in atherosclerosis:

1) SMC lineage tracing, SMC Col15a1 knockout and SMC lineage tracing SMC IL1R1 knockout mice exhibit nearly identical phenotypes after 18 weeks of Western diet feeding (Figures 3 and 13). Specifically, SMC Col15a1 knockout mouse lesions are very small ( $~ 30,000 \mu m^{2}$ lesion area) and fatty streak like in appearance in that they are macrophage-rich, SMC 
and collagen-poor, and devoid of a fibrous cap as compared to advanced wild type control lesions. Similarly, studies by Delphine Gomez in SMC IL $1 R$ knockout, SMC lineage tracing mice fed 18 weeks Western diet also show small lesions $\left(\sim 70,000 \mu \mathrm{m}^{2}\right.$ lesion area) and appear fatty streak like (Figures 3 and 13).

2) A net reduction in COL15A1 and IL1 $\beta$ levels within lesions results in similar changes to cell content and proliferation within lesions. Delphine has performed an intervention study in which she treats 18 week Western diet fed SMC lineage tracing mice with a mouse anti-IL1 $\beta$ antibody for 3 weeks. These animals show reduced SMC proliferation, increased macrophage proliferation, and reduced collagen content within the fibrous cap (Figure 13 and data not shown). Comparably, we observed a decrease in SMC proliferation, near significant increase in macrophage proliferation, and a decrease in collagen content in our SMC Col15a1 knockout mice after 18 weeks of Western diet (Figures 4A-F and 7D).

3) The Upstream Regulator Analysis of our in vivo RNA-seq from our SMC Col15a1 knockout and wild type lesions discovered a SMC-COL15A1 dependent inhibition of the IL1 $\beta$ pathway (Figure 9).

While these observations are highly intriguing, if and how COL15A1 is connected to the IL1R1 and/or IL1 $\beta$ within atherosclerosis will be challenging to ascertain. One possibility is that COL15A1 binds to either IL1 $\beta$ or IL1R1. To test this, we would need to have successful co-immunoprecipitation of COL15A1 with IL1 $\beta$ and/or the IL1R1 and vice versa from atherosclerotic aortas. Another way to 
begin to assess how IL1 $\beta$ or IL1R1 and COL15A1 may be connected is through a series of in vitro SMC experiments. Specifically, the first experiment would be to see if IL1 $\beta$ treatment in SMC induces Col15a1 expression. In tandem, it would be interesting to determine if IL1R1 expression is reduced with siRNA knockdown of Col15a1 or in Col15a1 knockout SMC. Based on the results of these culture experiments, a future direction may be to look at changes in SMC proliferation with manipulation of IL1 $\beta$ or COL15A1 levels given that both IL1 $\beta$ and COL15A1 levels appear to impact SMC proliferation in vivo. Lastly, it is also possible that the changes we see in vivo in response to a reduction in SMC COL15A1 and SMC IL1R1-mediated signaling in lesions are the consequence of secondary events that are shared between or that work in parallel in both models. Taken together, exploring the above association and observations represents an exciting prospective new direction of inquiry.

\subsection{Is COL15A1 likely to be involved in human plaque erosion events?}

Although plaque rupture accounts for the majority (between $55-60 \%$ ) of sudden coronary deaths, approximately $30-40 \%$ of sudden coronary deaths are the consequence of plaque erosion ${ }^{1}$. Plaque erosion is considered to be on the rise clinically, associated with non-ST elevation myocardial infarction, and prevalent in young or female patients ${ }^{50,51,188,189}$. Plaque erosion is characterized by the development of a thrombus on lesions with a denuded endothelium that contain an abundance of $\mathrm{ACTA} 2^{+}$cells, proteoglycans (identified by Movat) and non-fibrillar collagens (e.g. collagen type IV) at the luminal edge $\mathrm{e}^{1,31,49,50,190}$. It is 
important to know that though this is the definition of plaque erosion, it is unknown whether the $\mathrm{ACTA} 2^{+}$cells are of SMC or myeloid origin. Moreover, the use of Movat staining for identification of proteoglycans provides no information as to proteoglycan types in these lesions. However, as COL15A1 is a chondroitin sulfate proteoglycan ${ }^{24}$ and we have shown previously ${ }^{21}$ and in Chapter 2 that COL15A1 is produced by SMC, it might be intriguing to see if COL15A1 is abundant at the luminal edge of human plaque erosion lesions. Another future avenue of study might involve determining whether the COL15A1 SNP is a biomarker of atherosclerotic disease phenotypes. Previously, we identified from human aorta samples a COL15A1 SNP in human aorta samples that could be epigenetically regulated and was associated with age-related atherosclerotic disease risk ${ }^{21}$. However, we did not assess indices of lesion stability or lesion phenotype in this analysis. Recently, the Owens lab submitted a grant proposal in which the lab would collaborate with the foremost expert in atherosclerosis pathology, Renu Virmani. Dr. Virmani has at her disposal a large biobank of coronary atherosclerotic lesion samples pathologically characterized for plaque rupture or erosion with matching patient blood samples. Therefore, a prospective study might be to assess if there is an association between the COL15A1 SNP within patient blood samples and plaque rupture, erosion, or other indices of plaque stability. 
Figure 11

A) Collagen Matrix generated by Col15a1 KO SMC

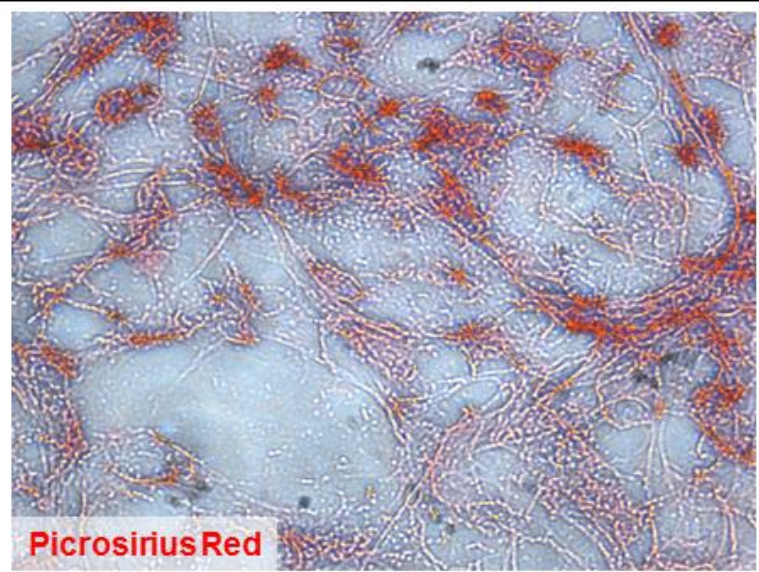

B) Col15a1 KO SMC re-plated on Collagen Matrix generated byCol15a1 KO SMC
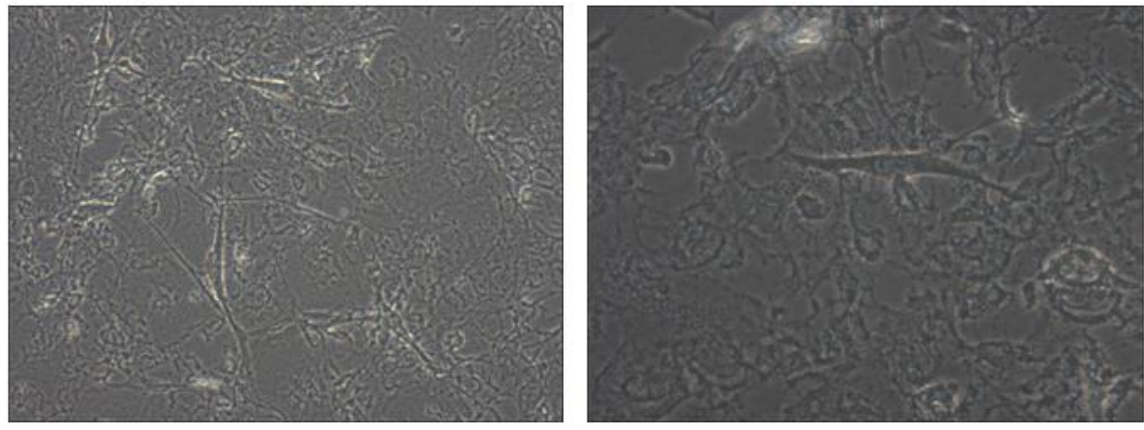
Figure 11: Preliminary data on SMC Col15a1 knockout (KO) cells generating a collagen matrix

SMC Col15a1 knockout cells were plated at a density of $1.0 \times 10^{4}$ cells $/ \mathrm{cm}^{2}$ and treated with 10\% serum every 2 days for 3 weeks. At 3 weeks, cells were treated with a calcium chelating solution (10mM EDTA, 10mM EGTA in sterile 1XPBS solution) and placed in a $37^{\circ} \mathrm{C}$ incubator. Cell detachment was checked every hour via light microscope with detachment taking between 3-5 hours on average; too much calcium chelation would cause matrix degradation. When cells appeared detached from the underlying matrix, the cells were washed thrice with sterile 1XPBS. Cells were then treated with sterile $\mathrm{H}_{2} \mathrm{O}$ for $3-5 \mathrm{~min}$ to induce cell death by hypotonic shock and maintain the matrix put down by the cells. Cells were then washed thrice gently with 1X PBS to remove cell debris and keep the underlying matrix intact. The matrix was then either immediately A) stained with Picrosirius red to detect collagen in the matrix (in red) or B) plated with new Col15a1 knockout SMC which were allowed 24 hours to adhere. Images in B) show Col15a1 knockout SMC can bind and spread on the matrix. We made the observation that there was a slight bias in attachment for non-matrix containing regions of the plaque and that the matrix was digested 48 hours after re-plating of cells. Unfortunately, results were inconsistent. 
Figure 12

A) Adapted from Gomez et al. Nat. Methods (2013)

3 PLA Signal

入入 PLA $2^{\text {nd }} A b$

入入 Primary Ab

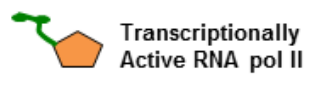

+ Biotin

Ene Probe

B)
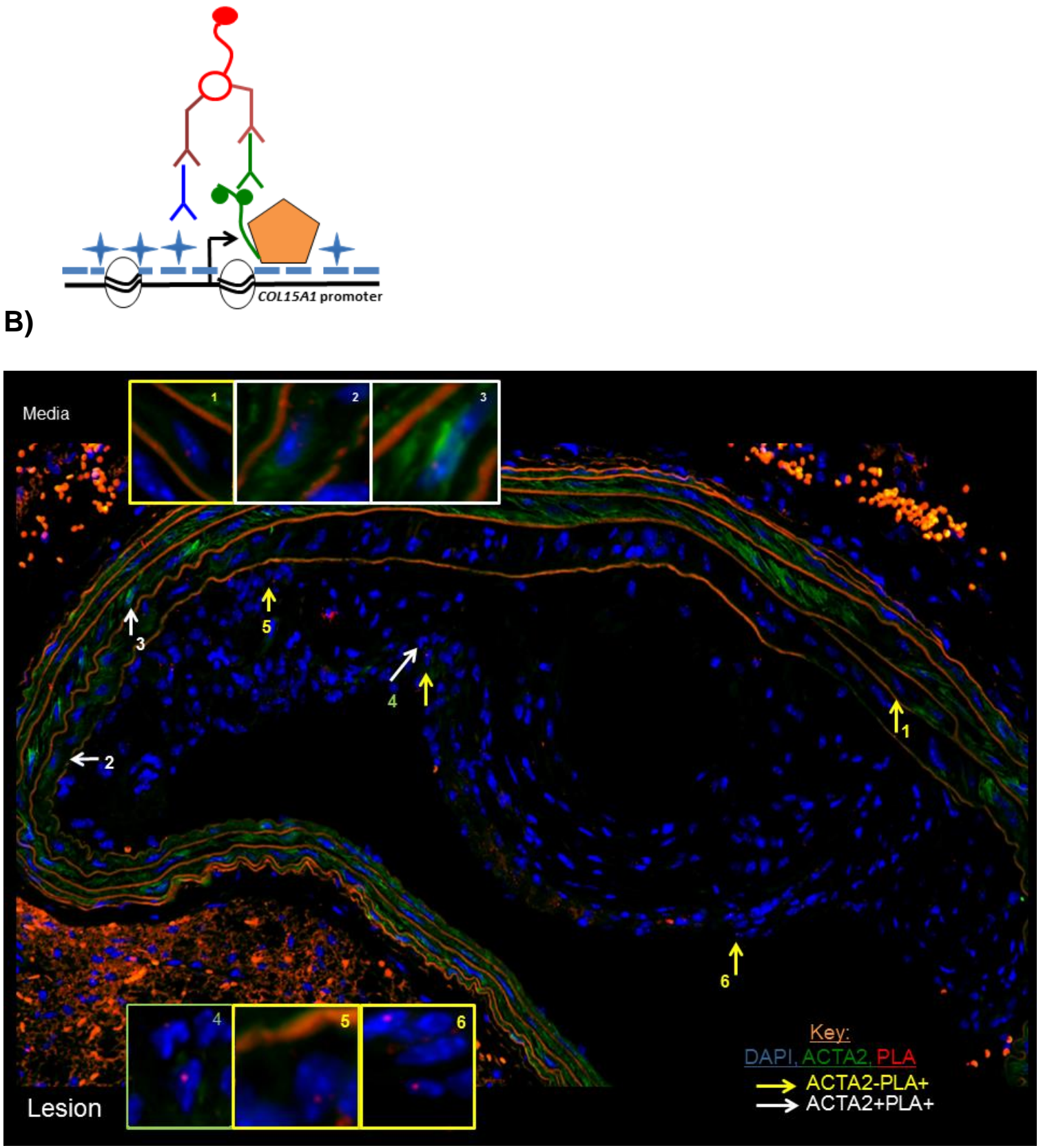
Figure 12: In situ hybridization-proximity ligation assay (ISH-PLA) of RNA Polymerase II (RNA Pol II) bound to Col15a1 promoter.

A) Schematic of ISH-PLA adapted from Gomez et $a I^{16}$. B) An atherosclerotic lesion from an 18 week Western diet fed SMC lineage tracing mouse stained with DAPI (nuclear stain, blue), ACTA2 (SMC marker gene, green), and ISH-PLA (red dot). The ISH-PLA of RNA Pol II bound to Col15a1 promoter (PLA+) is indicated by red dots in the cell nucleus. There are $A C T A 2^{+} \operatorname{PLA}^{+}$(white arrows) and $\mathrm{ACTA}^{-} \mathrm{PLA}^{+}$(yellow arrows) cells in both the media (zoom top, 1-3) and the lesion (zoom bottom, 4-6). Unfortunately, staining for SMC lineage tracing mark YFP did not work in this experiment. We can conclude that both $\mathrm{ACTA}^{+}$and ACTA2 $^{-}$cells within the media and lesion can express Col15a1. 
Figure 13

A)

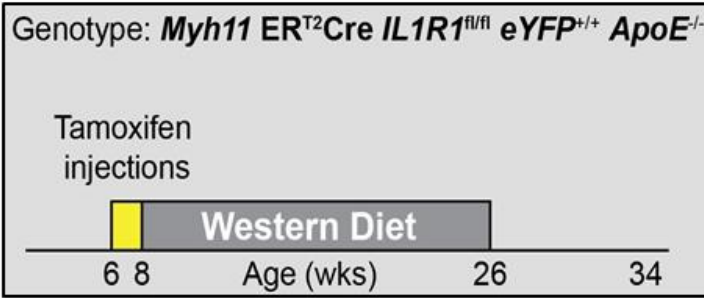

SMC IL1R1wt/wt

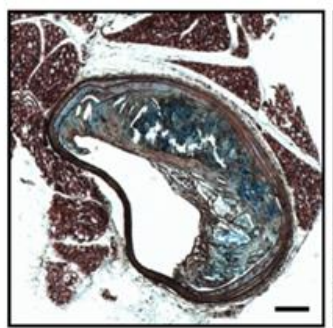

SMC-IL1R1 $1 / \Delta$

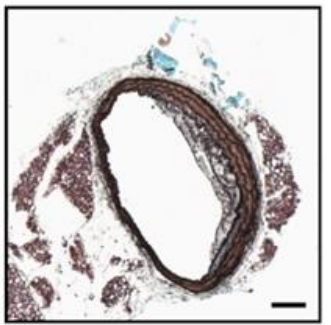

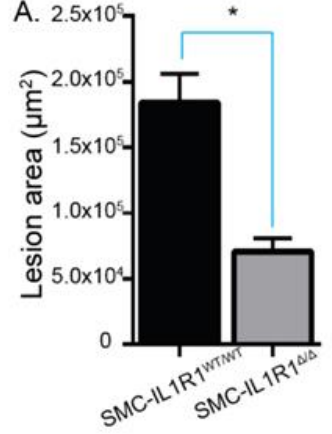

B)

Genotype: Myh11 ER ${ }^{\mathrm{T} 2} \mathrm{Cre}$ eYFP ${ }^{+/}$ApoE

Anti-IL $1 \beta \mathrm{Ab}$ or IgG Contro $10 \mathrm{mg} / \mathrm{kg}$

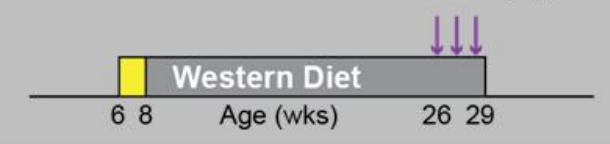

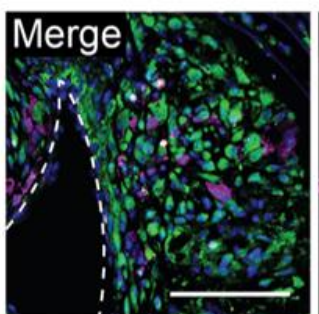

Merge

순
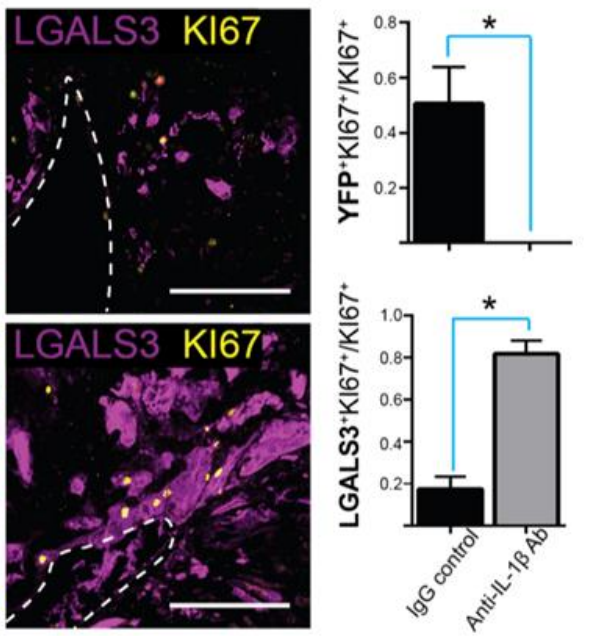
Figure 13: Preliminary data from SMC IL1R1 knockout atheroprevention and anti-IL1 $\beta$ atherointervention studies.

A) SMC lineage tracing, SMC IL1R1 knockout mice fed 18 weeks Western diet exhibit drastically smaller lesions than wild type controls, but are similar to those seen in SMC Col15a1 knockout animals. B) SMC lineage tracing mice treated with anti-IL1 $\beta$ antibody for 3 weeks show reduced SMC proliferation $\left(\mathrm{YFP}^{+} \mathrm{KI}^{+} 7^{+}\right)$ and increased LGALS3 $^{+}$cell proliferation $\left(\mathrm{LGALS3}^{+} \mathrm{KI} 67^{+}\right.$) cells per total proliferating $\left(\mathrm{KI} 67^{+}\right)$cells in the fibrous cap. 
Figure 14

Downregulated with SMC Col15a1 knockout Upregulated with SMC Col15a1 knockout

\begin{tabular}{|l|r|r|}
\hline \multicolumn{1}{|c|}{ Gene } & log $_{2}$ FoldChange & p-adj \\
\hline Gbp2b & -1.959571198 & $1.54 \mathrm{E}-07$ \\
Col15a1 & -1.206130662 & 0.00237275 \\
\hline Tnfsf15 & -1.194271825 & 0.000176851 \\
\hline Tubb1 & -1.135945936 & 0.032303005 \\
Ppbp & -1.115719296 & 0.039541612 \\
Prg4 & -1.073088467 & 0.016342193 \\
E2f8 & -1.03538658 & 0.049893871 \\
Pvrl4 & -1.005317307 & 0.009671497 \\
Apoe & -0.914606793 & 0.000634476 \\
Arl4c & -0.897166611 & 0.008202291 \\
Ccr5 & -0.888399497 & 0.018772964 \\
Aif1 & -0.888148986 & 0.037160088 \\
Susd3 & -0.852385906 & 0.004305571 \\
Neur13 & -0.82365406 & 0.010924697 \\
Fabp5 & -0.808229565 & 0.006477145 \\
Coro1a & -0.795748577 & $3.98 \mathrm{E}-05$ \\
\hline Kif26b & -0.781638194 & 0.011882494 \\
Hist2h2bb & -0.713018461 & 0.010841568 \\
Sdc3 & -0.695711272 & 0.037478944 \\
Slc7a7 & -0.623067332 & 0.020685815 \\
\hline
\end{tabular}

\begin{tabular}{|l|r|r|}
\hline \multicolumn{1}{|c|}{ Gene } & log $_{2}$ FoldChange & \multicolumn{1}{c|}{ p-adj } \\
\hline Uqcr10 & 0.471025834 & 0.04605665 \\
\hline Idh3a & 0.671622541 & 0.009351205 \\
Sucla2 & 0.699561875 & 0.000466221 \\
Letmd1 & 0.700382877 & 0.006477145 \\
\hline Rgcc & 0.705168918 & 0.04605665 \\
\hline Atp1a2 & 0.715566551 & 0.004981279 \\
\hline Tmem143 & 0.715667501 & 0.006425837 \\
Cecr2 & 0.857903497 & 0.039541612 \\
Perm1 & 0.872173696 & 0.0028192 \\
Slc4a4 & 0.873288123 & 0.019797361 \\
Ppara & 0.877780318 & 0.010376766 \\
Npy1r & 0.930417455 & 0.003442229 \\
Bche & 0.935900249 & 0.000317873 \\
Fabp3 & 0.973814496 & 0.045840034 \\
Gabrq & 0.98315297 & 0.004305571 \\
Mlxipl & 1.00206012 & 0.009351205 \\
Fam13a & 1.00621795 & 0.046897187 \\
Wnt2b & 1.048634457 & $5.42 \mathrm{E}-05$ \\
Kcna5 & 1.110122427 & 0.011050322 \\
\hline Trpc5 & 1.178440368 & 0.018094649 \\
Aspg & 1.198174282 & 0.000349156 \\
Scn4b & 1.232295115 & 0.009375936 \\
\hline Timp4 & 1.365151425 & $1.11 \mathrm{E}-06$ \\
\hline A530050N04Rik & 1.365869082 & 0.001605717 \\
Slc27a2 & 1.529415382 & 0.000113895 \\
\hline
\end{tabular}


Figure 14: In vivo RNA-seq data of list of genes upregulated and downregulated with SMC Col15a1 knockout.

The carotid arteries, aortic arch, and brachiocephalic artery from SMC Col15a1 knockout and wild type 18 week Western diet fed animals were analyzed via RNA-seq. The gene list in this figure show genes from the RNA-seq first sorted based on adjusted $p$-value ( $p$-adj) and then subsequently sorted by $\log _{2}$ FoldChange. Red indicates genes upregulated with SMC Col15a1 knockout and blue indicates genes downregulated with SMC Col15a1 knockout. 


\section{CHAPTER 4:}

\section{References}


1. Virmani, R., Kolodgie, F. D., Burke, A. P., Farb, A. \& Schwartz, S. M. Lessons From Sudden Coronary Death. Arter. Thromb Vasc Biol 20, 1262$1275(2000)$.

2. Finn, A. V, Nakano, M., Narula, J., Kolodgie, F. D. \& Virmani, R. History of Discovery Concept of Vulnerable / Unstable Plaque. 1282-1293 (2010). doi:10.1161/ATVBAHA.108.179739

3. WHO. Global status report on noncommunicable diseases 2014. World Health 176 (2014). doi:ISBN 9789241564854

4. Barquera, S. et al. Global Overview of the Epidemiology of Atherosclerotic Cardiovascular Disease. Arch. Med. Res. 46, 328-338 (2015).

5. Wang, H. et al. Global, regional, and national life expectancy, all-cause mortality, and cause-specific mortality for 249 causes of death, 1980-2015: a systematic analysis for the Global Burden of Disease Study 2015. Lancet 388, 1459-1544 (2016).

6. Kassebaum, N. J. et al. Global, regional, and national disability-adjusted life-years (DALYs) for 315 diseases and injuries and healthy life expectancy (HALE), 1990-2015: a systematic analysis for the Global Burden of Disease Study 2015. Lancet 388, 1603-1658 (2016).

7. Davies, M. J., Richardson, P. D., Woolf, N., Katz, D. R. \& Mann, J. Risk of thrombosis in human atherosclerotic plaques : role of extracellular lipid, macrophage, and smooth muscle cell content. Br. Heart J. 69, 377-381 (1993).

8. Libby, P., Ridker, P. M. \& Hansson, G. K. Progress and challenges in translating the biology of atherosclerosis. Nature 473, 317-25 (2011).

9. Virmani, R., Burke, A. P., Farb, A. \& Kolodgie, F. D. Pathology of the Vulnerable Plaque. J. Am. Coll. Cardiol. 47, C13-C18 (2006).

10. Fishbein, M. C. The vulnerable and unstable atherosclerotic plaque. Cardiovasc. Pathol. Off. J. Soc. Cardiovasc. Pathol. 19, 6-11 (2010).

11. Amento, E. P., Ehsani, N., Palmer, H. \& Libby, P. Cytokines and growth factors positively and negatively regulate interstitial collagen gene expression in human vascular smooth muscle cells. Arter. Thromb Vasc Biol 11, 1223-1230 (1991).

12. Cherepanova, O. A. et al. Oxidized phospholipids induce type VIII collagen expression and vascular smooth muscle cell migration. Circ. Res. 104, 609-618 (2009). 
13. Adiguzel, E., Ahmad, P. J., Franco, C. \& Bendeck, M. P. Collagens in the progression and complications of atherosclerosis. Vasc. Med. 14, 73-89 (2009).

14. Cherepanova, O. A. et al. Activation of the pluripotency factor OCT4 in smooth muscle cells is atheroprotective. Nat. Med. 1-12 (2016). doi: $10.1038 / \mathrm{nm} .4109$

15. Shankman, L. S. et al. KLF4-dependent phenotypic modulation of smooth muscle cells has a key role in atherosclerotic plaque pathogenesis. Nat. Med. 21, 628-637 (2015).

16. Gomez, D., Shankman, L. S., Nguyen, A. T. \& Owens, G. K. Detection of histone modifications at specific gene loci in single cells in histological sections. Nat. Methods 10, 171-7 (2013).

17. Caplice, N. M. et al. Smooth muscle cells in human coronary atherosclerosis can originate from cells administered at marrow transplantation. Proc. Natl. Acad. Sci. U. S. A. 100, 4754-9 (2003).

18. Rong, J. X., Shapiro, M., Trogan, E. \& Fisher, E. a. Transdifferentiation of mouse aortic smooth muscle cells to a macrophage-like state after cholesterol loading. Proc. Natl. Acad. Sci. U. S. A. 100, 13531-13536 (2003).

19. Chen, P. Y. et al. Endothelial-to-mesenchymal transition drives atherosclerosis progression. J. Clin. Invest. 125, 4514-4528 (2015).

20. Feil, S. et al. Transdifferentiation of vascular smooth muscle cells to macrophage-like cells during atherogenesis. Circ. Res. 115, 662-667 (2014).

21. Connelly, J. J. et al. Epigenetic regulation of COL15A1 in smooth muscle cell replicative aging and atherosclerosis. Hum. Mol. Genet. 22, 5107-5120 (2013).

22. Myers, J. C., Kivirikko, S., Gordon, M. K., Dion, A. S. \& Pihlajaniemi, T. Identification of a previously unknown human collagen chain, alpha $1(\mathrm{XV})$, characterized by extensive interruptions in the triple-helical region. Proc. Natl. Acad. Sci. U. S. A. 89, 10144-10148 (1992).

23. Amenta, P. S. et al. Proteoglycan-collagen $X V$ in human tissues is seen linking banded collagen fibers subjacent to the basement membrane. $J$. Histochem. Cytochem. 53, 165-176 (2005). 
24. Li, D., Clark, C. C. \& Myers, J. C. Basement membrane zone type XV collagen is a disulfide-bonded chondroitin sulfate proteoglycan in human tissues and cultured cells. J. Biol. Chem. 275, 22339-22347 (2000).

25. Rasi, K. et al. Collagen XV Is Necessary for Modeling of the Extracellular Matrix and Its Deficiency Predisposes to Cardiomyopathy. Circ. Res. 107, 1241-1252 (2010).

26. Eklund, L. et al. Lack of type XV collagen causes a skeletal myopathy and cardiovascular defects in mice. Proc. Natl. Acad. Sci. U. S. A. 98, 11941199 (2001).

27. (CDC), C. for D. C. and P. Leading Causes of Death: United States. Available at: https://www.cdc.gov/nchs/fastats/leading-causes-ofdeath.htm.

28. (CDC), C. for D. C. and P. Heart Disease Facts. Available at: https://www.cdc.gov/heartdisease/facts.htm.

29. Mozaffarian, D. et al. Heart Disease and Stroke Statistics--2015 Update: A Report From the American Heart Association. Circulation 131, (2015).

30. NIH National Heart Lung and Blood Institute. What is Atherosclerosis? Available at: https://www.nhlbi.nih.gov/health/healthtopics/topics/atherosclerosis.

31. Bentzon, J. F., Otsuka, F., Virmani, R. \& Falk, E. Mechanisms of plaque formation and rupture. Circ. Res. 114, 1852-1866 (2014).

32. Yahagi, K. et al. Pathophysiology of native coronary, vein graft, and instent atherosclerosis. Nat. Rev. Cardiol. 13, 79-98 (2016).

33. Pelisek, Jaroslav, Eckstein, Hans-Henning, and Zernecke, A. Pathophysiological Mechanisms of Carotid Plaque Vulnerability: Impact on Ischemic Stroke. Achivum Immunol. Ther. Exp. 60, 431-442 (2012).

34. NIH National Heart Lung and Blood Institute. Who is at Risk for Atherosclerosis? Available at: https://www.nhlbi.nih.gov/health/healthtopics/topics/atherosclerosis/atrisk.

35. Palinski, W. \& Napoli, C. The fetal origins of atherosclerosis: maternal hypercholesterolemia, and cholesterol-lowering or antioxidant treatment during pregnancy influence in utero programming and postnatal susceptibility to atherogenesis. FASEB J. 16, 1348-60 (2002). 
36. Fryar, C. D., Chen, T.-C. \& Li, X. Prevalence of uncontrolled risk factors for cardiovascular disease: United States, 1999-2010. NCHS Data Brief 1-8 (2012).

37. NIH-National Heart Lung and Blood Institute. Who is at Risk for Stroke? Available at: https://www.nhlbi.nih.gov/health/healthtopics/topics/stroke/atrisk.

38. NIH-National Heart Lung and Blood Institute. Who is at Risk for Heart Disease. Available at: https://www.nhlbi.nih.gov/health/healthtopics/topics/cad/atrisk.

39. Centers for Disease Control and Prevention, C. Heart Disease Risk Factors. Available at: https://www.cdc.gov/heartdisease/risk_factors.htm.

40. NIH National Heart Lung and Blood Institute. How is Atherosclerosis Treated? Available at: https://www.nhlbi.nih.gov/health/healthtopics/topics/atherosclerosis/treatment.

41. Tabas, I., Garcia-Cardena, G. \& Owens, G. K. Recent insights into the cellular biology of atherosclerosis. J. Cell Biol. 209, 13-22 (2015).

42. Gomez, D. \& Owens, G. K. Smooth muscle cell phenotypic switching in atherosclerosis. Cardiovasc. Res. 95, 156-64 (2012).

43. Finn, A. V., Nakano, M., Narula, J., Kolodgie, F. D. \& Virmani, R. Concept of vulnerable/unstable plaque. Arterioscler. Thromb. Vasc. Biol. 30, 12821292 (2010).

44. Kher, N. \& Marsh, J. D. Pathobiology of atherosclerosis--a brief review. Semin. Thromb. Hemost. 30, 665-672 (2004).

45. Shah, P. K. Mechanisms of plaque vulnerability and rupture. J. Am. Coll. Cardiol. 41, 15S-22S (2003).

46. Lendon, C. L., Davies, M. J., Born, G. V. R. \& Richardson, P. D. Atherosclerotic plaque caps are locally weakened when macrophages density is increased. Atherosclerosis 87, 87-90 (1991).

47. Majesky, M. W. Mouse model for atherosclerotic plaque rupture. Circulation 105, 2010-2011 (2002).

48. Schwartz, S. M., Galis, Z. S., Rosenfeld, M. E. \& Falk, E. Plaque rupture in humans and mice. Arterioscler. Thromb. Vasc. Biol. 27, 705-713 (2007). 
49. Farb, A. et al. Coronary Plaque Erosion Without Rupture Into a Lipid Core. Circulation 93, 1354 LP-1363 (1996).

50. Libby, P. \& Pasterkamp, G. Requiem for the 'vulnerable plaque'. Eur. Heart J. 36, 2984-2987 (2015).

51. Jia, H. et al. In vivo diagnosis of plaque erosion and calcified nodule in patients with acute coronary syndrome by intravascular optical coherence tomography. J. Am. Coll. Cardiol. 62, 1748-1758 (2013).

52. Alexander, M. R. \& Owens, G. K. Epigenetic Control of Smooth Muscle Cell Differentiation and Phenotypic Switching in Vascular Development and Disease. Annu. Rev. Physiol. (2011). doi:10.1146/annurev-physiol-012110142315

53. Owens, G. K., Kumar, M. S. \& Wamhoff, B. R. Molecular regulation of vascular smooth muscle cell differentiation in development and disease. Physiol. Rev. 84, 767-801 (2004).

54. Yang, S. N. Y. et al. Transforming growth factor-beta regulation of proteoglycan synthesis in vascular smooth muscle: Contribution to lipid binding and accelerated atherosclerosis in diabetes. J. Diabetes 2, 233242 (2010).

55. Wick, Georg, Grundtman, C. Inflammation and Atherosclerosis. (2012).

56. Pidkovka, N. A. et al. Oxidized phospholipids induce phenotypic switching of vascular smooth muscle cells in vivo and in vitro. Circ. Res. 101, 792801 (2007).

57. Iwata, H. et al. Bone Marrow-Derived Cells Contribute to Vascular Inflammation but Do Not Differentiate Into Smooth Muscle Cell Lineages. Circulation 122, 2048-2057 (2010).

58. Allahverdian, S., Chehroudi, A. C., McManus, B. M., Abraham, T. \& Francis, G. A. Contribution of intimal smooth muscle cells to cholesterol accumulation and macrophage-like cells in human atherosclerosis. Circulation 129, 1551-1559 (2014).

59. Albarran-Juarez, J., Kaur, H., Grimm, M., Offermanns, S. \& Wettschureck, $\mathrm{N}$. Lineage tracing of cells involved in atherosclerosis. Atherosclerosis 16, 445-453 (2016). 
60. Chappell, J. et al. Extensive Proliferation of a Subset of Differentiated, Yet Plastic, Medial Vascular Smooth Muscle Cells Contribute to Neointimal Formation in Mouse Injury and Atherosclerosis Models. Circ. Res. 119, (2016).

61. Sata, M. et al. Hematopoietic stem cells differentiate into vascular cells that participate in the pathogenesis of atherosclerosis. Nat. Med. 8, 403-409 (2002).

62. Iwata, $\mathrm{H}$. et al. Bone marrow-derived cells contribute to vascular inflammation but do not differentiate into smooth muscle cell lineages. Circulation 122, 2048-2057 (2010).

63. Benditt, E. P. \& Benditt, J. M. Evidence for a monoclonal origin of human atherosclerotic plaques. Proc. Natl. Acad. Sci. U. S. A. 70, 1753-6 (1973).

64. Thomas, W. A., Florentin, R. A., Reiner, J. M., Lee, W. M. \& Lee, K. T. Alterations in population dynamics of arterial smooth muscle cells during atherogenesis. Exp. Mol. Pathol. 24, 244-260 (1976).

65. Vengrenyuk, Y. et al. Cholesterol loading re-programs the miR-143/145myocardin axis to convert aortic smooth muscle cells to a dysfunctional macrophage-like phenotype. Arterioscler. Thromb. Vasc. Biol. 35, 535-546 (2015).

66. Mouw, J. K., Ou, G. \& Weaver, V. M. Extracellular matrix assembly: a multiscale deconstruction. Nat. Rev. Mol. Cell Biol. 15, 771-785 (2014).

67. Ricard-Blum, S. The Collagen Family. Cold Spring Harb. Perspect. Biol. 3, 1-19 (2011).

68. Orr, A. W. et al. Molecular mechanisms of collagen isotype-specific modulation of smooth muscle cell phenotype. Arterioscler. Thromb. Vasc. Biol. 29, 225-231 (2009).

69. Plenz, G. A. M., Deng, M. C., Robenek, H. \& Vo, W. Vascular collagens : spotlight on the role of type VIII collagen in atherogenesis. Atherosclerosis 166, 1-11 (2003).

70. Rekhter, M. D. Collagen synthesis in atherosclerosis: too much and not enough. Cardiovasc. Res. 41, 376-84 (1999).

71. Kalluri, R. Basement membranes: structure, assembly and role in tumour angiogenesis. Nat. Rev. Cancer 3, 422-433 (2003). 
72. Ortega, N. \& Werb, Z. New functional roles for non-collagenous domains of basement membrane collagens. J. Cell Sci. 115, 4201-4214 (2002).

73. Yurchenco, P. D. Basement membranes: Cell scaffoldings and signaling platforms. Cold Spring Harb. Perspect. Biol. 3, 1-27 (2011).

74. Smith, E. B. The Influence of Age and Atherosclerosi on the Chemistry of Aortic Intima. 2. Collagen and Mucopolysaccharides. J. Atheroscler. Res. 5, 241-248 (1965).

75. Shekhonin, B. V, Domogatsky, S. P., Muzykantov, V. R., Idelson, G. L. \& Rukosuev, V. S. Distribution of type I, III, IV and V collagen in normal and atherosclerotic human arterial wall: immunomorphological characteristics. Coll. Relat. Res. 5, 355-368 (1985).

76. Murata, K., Motayama, T. \& Kotake, C. Collagen types in various layers of the human aorta and their changes with the atherosclerotic process. Atherosclerosis 60, 251-262 (1986).

77. Katsuda, S. et al. Collagens in human atherosclerosis. Immunohistochemical analysis using collagen type-specific antibodies. Arterioscler. Thromb. Vasc. Biol. 12, 494-502 (1992).

78. Durgin, B. G. et al. Smooth muscle cell-specific deletion of Col15a1 unexpectedly leads to impaired 1 development of advanced atherosclerotic lesions 2 3. Am. J. Physiol. - Hear. Circ. Physiol. 4403, (2017).

79. Burleigh, MC; Briggs, AD; Lendon, Cl; Davies, MJ;Born, GV; Richardson, P. Collagen types I and III, collagen content, GAGs and mechanical strength of human atherosclerotic plaque caps; span-wise variations. Atherosclerosis 96, 71-81 (1992).

80. Arroyo, L. H. \& Lee, R. T. Mechanisms of plaque rupture. Cardiovasc. Res. 41, 369-375 (1999).

81. Arroyo, L. H. \& Lee, R. T. Mechanisms of plaque rupture: Mechanical and biologic interactions. Cardiovasc. Res. 41, 369-375 (1999).

82. Harburger, D. S. \& Calderwood, D. A. Integrin signalling at a glance. J. Cell Sci. 122, 159-163 (2009).

83. Leitinger, B. Discoidin domain receptor functions in physiological and pathological conditions. Int. Rev. Cell Mol. Biol. 310, 39-87 (2014).

84. Finney, A. C., Stokes, K. Y., Pattillo, C. B. \& Orr, A. W. Integrin signaling in atherosclerosis. Cell. Mol. Life Sci. 0, 0 (2017). 
85. Franco, C., Ahmad, P. J., Hou, G., Wong, E. \& Bendeck, M. P. Increased cell and matrix accumulation during atherogenesis in mice with vessel wallspecific deletion of discoidin domain receptor 1. Circ. Res. 106, 1775-1783 (2010).

86. Franco, C. et al. Discoidin domain receptor 1 on bone marrow-derived cells promotes macrophage accumulation during atherogenesis. Circ. Res. 105, 1141-1148 (2009).

87. Franco, C. et al. Discoidin domain receptor 1 (Ddr1) deletion decreases atherosclerosis by accelerating matrix accumulation and reducing inflammation in low-density lipoprotein receptor-deficient mice. Circ. Res. 102, 1202-1211 (2008).

88. Ricard-Blum, S. \& Salza, R. Matricryptins and matrikines: Biologically active fragments of the extracellular matrix. Exp. Dermatol. 23, 457-463 (2014).

89. Ricard-Blum, S. \& Faye, C. Collagens associated with basement membranes and their matricryptins. J. Soc. Biol. 199, 321-328 (2005).

90. Maquart, F. X., Pasco, S., Ramont, L., Hornebeck, W. \& Monboisse, J. C. An introduction to matrikines: Extracellular matrix-derived peptides which regulate cell activity - Implication in tumor invasion. Crit. Rev. Oncol. Hematol. 49, 199-202 (2004).

91. Colorado, P. C. et al. Anti-angiogenic cues from vascular basement membrane collagen. Cancer Res. 60, 2520-2526 (2000).

92. Mundel, T. M. \& Kalluri, R. Type IV collagen-derived Angiogenesis Inhibitors. Microvasc. Res. 74, 85-89 (2007).

93. Fukumoto, Y. et al. Genetically determined resistance to collagenase action augments interstitial collagen accumulation in atherosclerotic plaques. Circulation 110, 1953-1959 (2004).

94. Moulton, K. S. et al. Loss of Collagen XVIII Enhances Neovascularization and Vascular Permeability in Atherosclerosis. Circulation 110, 1330-1337 (2004).

95. Lopes, J. et al. Type VIII Collagen Mediates Vessel Wall Remodeling after Arterial Injury and Fibrous Cap Formation in Atherosclerosis. The American Journal of Pathology 182, 2241-2253 (2013). 
96. Schlumberger, W., Thie, M., Rauterberg, J. \& Robenek, H. Collagen Synthesis in Cultured Aortic Smooth Muscle Cells. Atheroscler. Thromb. Vasc. Biol. 11, 1660-1667 (1991).

97. Holycross, B. J., Blank, R. S., Thompson, M. M., Peach, M. J. \& Owens, G. K. Platelet-derived growth factor-BB-induced suppression of smooth muscle cell differentiation. Circ Res 71, 1525-1532 (1992).

98. Li, X. et al. Suppression of smooth-muscle alpha-actin expression by platelet-derived growth factor in vascular smooth-muscle cells involves Ras and cytosolic phospholipase A2. Biochem. J. 327, 709-716 (1997).

99. Corjay, M. H., Thompson, M. M., Lynchgli, K. R. \& Owenssii, G. K. Differential Effect of Platelet-derived Growth Factor- Versus Seruminduced Growth on Smooth Muscle a-Actin and Nonmuscle alpha -Actin mRNA Expression in Cultured Rat Aortic Smooth Muscle Cells *. J. Biol. Chem. 264, 10501-10506 (1989).

100. Adiguzel, E. et al. Migration and growth are attenuated in vascular smooth muscle cells with type VIII collagen-null alleles. Arterioscler. Thromb. Vasc. Biol. 26, 56-61 (2006).

101. Koyama, H., Raines, E. W., Bornfeldt, K. E., Roberts, J. M. \& Ross, R. Fibrillar collagen inhibits arterial smooth muscle proliferation through regulation of Cdk2 inhibitors. Cell 87, 1069-1078 (1996).

102. Barrett, S. R. H., Sutcliffe, M. P. F., Howarth, S., Li, Z. Y. \& Gillard, J. H. Experimental measurement of the mechanical properties of carotid atherothrombotic plaque fibrous cap. J. Biomech. 42, 1650-1655 (2009).

103. Myers, J. C., Dion, A. S., Abraham, V. \& Amenta, P. S. Type XV collagen exhibits a widespread distribution in human tissues but a distinct localization in basement membrane zones. Cell Tissue Res. 286, 493-505 (1996).

104. Myers, J. C. et al. The molecular structure of human tissue type XV presents a unique conformation among the collagens. Biochem. J. 404, 535-544 (2007).

105. Hägg, P. M., Muona, A., Liétard, J., Kivirikko, S. \& Pihlajaniemi, T. Complete exon-intron organization of the human gene for the alpha1 chain of type XV collagen (COL15A1) and comparison with the homologous COL18A1 gene. J. Biol. Chem. 273, 17824-17831 (1998).

106. Clementz, A. G. et al. Collagen XV inhibits epithelial to mesenchymal transition in pancreatic adenocarcinoma cells. PLoS One 8, e72250 (2013). 
107. Clementz, A. G. \& Harris, A. Collagen XV: Exploring its Structure and Role within the Tumor Microenvironment. Mol. Cancer Res. 11, 1481-1486 (2013).

108. Kivirikko, S. et al. Primary structure of the alpha 1 chain of human type XV collagen and exon-intron organization in the 3 ' region of the corresponding gene. J. Biol. Chem. 269, 4773-4779 (1994).

109. Mohammadi, M., Olsen, S. K. \& Goetz, R. A protein canyon in the FGFFGF receptor dimer selects from an a la carte menu of heparan sulfate motifs. Curr. Opin. Struct. Biol. 15, 506-516 (2005).

110. Hynes, R. O. Extracellular matrix: not just pretty fibrils. Science 326, 12161219 (2009).

111. Tabas, I., Williams, K. J. \& Boren, J. Subendothelial lipoprotein retention as the initiating process in atherosclerosis: Update and therapeutic implications. Circulation 116, 1832-1844 (2007).

112. Williams, K. J. Arterial wall chondroitin sulfate proteoglycans: diverse molecules with distinct roles in lipoprotein retention and atherogenesis. Curr. Opin. Lipidol. 12, 477-487 (2001).

113. Mutolo, M. J. et al. Tumor suppression by collagen XV is independent of the restin domain. Matrix Biol. 31, 285-9 (2012).

114. Xu, R. et al. Mouse restin inhibits bovine aortic endothelial cell proliferation and causes cell apoptosis. Sheng Wu Hua Xue Yu Sheng Wu Wu Li Xue Bao (Shanghai). 34, 138-142 (2002).

115. Ramchandran, R. et al. Antiangiogenic activity of restin, NC10 domain of human collagen XV: comparison to endostatin. Biochem. Biophys. Res. Commun. 255, 735-739 (1999).

116. John, H., Radtke, K., Ständker, L. \& Forssmann, W.-G. Identification and characterization of novel endogenous proteolytic forms of the human angiogenesis inhibitors restin and endostatin. Biochim. Biophys. Acta 1747, 161-170 (2005).

117. Tomono, Y. et al. Epitope-defined monoclonal antibodies against multiplexin collagens demonstrate that type XV and XVIII collagens are expressed in specialized basement membranes. Cell Struct. Funct. 27, 920 (2002).

118. Rygh, C. B. et al. Image-based assessment of microvascular function and structure in collagen XV- and XVIII-deficient mice. J. Physiol. 592, 325-36 (2014). 
119. Rasi, K. et al. Lack of collagen $X V$ impairs peripheral nerve maturation and, when combined with laminin-411 deficiency, leads to basement membrane abnormalities and sensorimotor dysfunction. J. Neurosci. 30, 14490-14501 (2010).

120. Guillon, E., Bretaud, S. \& Ruggiero, F. Slow Muscle Precursors Lay Down a Collagen XV Matrix Fingerprint to Guide Motor Axon Navigation. J. Neurosci. 36, 2663-2676 (2016).

121. Harris, A., Harris, H. \& Hollingsworth, M. A. Complete suppression of tumor formation by high levels of basement membrane collagen. Mol. Cancer Res. MCR 5, 1241-1245 (2007).

122. Zaferani, A. et al. Basement Membrane Zone Collagens XV and XVIII / Proteoglycans Mediate Leukocyte Influx in Renal Ischemia / Reperfusion. PLoS One 9, e106732 (2014).

123. Klein, L. W. Clinical implications and mechanisms of plaque rupture in the acute coronary syndromes. Am. Heart Hosp. J. 3, 249-255 (2005).

124. Cheng, G. C., Loree, H. M., Kamm, R. D., Fishbein, M. C. \& Lee, R. T. Distribution of circumferential stress in ruptured and stable atherosclerotic lesions. A structural analysis with histopathological correlation. Circulation 87, 1179-1187 (1993).

125. Ross, R., Wight, T. N., Strandness, E. \& Thiele, B. Human atherosclerosis. I. Cell constitution and characteristics of advanced lesions of the superficial femoral artery. Am. J. Pathol. 114, 79-93 (1984).

126. Barnes, M. J. \& Farndale, R. W. Collagens and atherosclerosis. Exp. Gerontol. 34, 513-525 (1999).

127. Yurdagul, A., Finney, A. C., Woolard, M. D. \& Orr, A. W. The arterial microenvironment: the where and why of atherosclerosis. Biochem. J. 473, 1281-1295 (2016).

128. Andreeva, E. R., Pugach, I. M. \& Orekhov, A. N. Subendothelial smooth muscle cells of human aorta express macrophage antigen in situ and in vitro. Atherosclerosis 135, 19-27 (1997).

129. Wirth, A. et al. G12-G13-LARG-mediated signaling in vascular smooth muscle is required for salt-induced hypertension. Nat. Med. 14, 64-68 (2008). 
130. Vooijs, M., Jonkers, J. \& Berns, A. A highly efficient ligand-regulated Cre recombinase mouse line shows that LoxP recombination is position dependent. EMBO Rep. 2, 292-297 (2001).

131. Billaud, M. et al. Characterization of the Thoracodorsal Artery: Morphology and Reactivity. Microcirculation 19, 360-372 (2012).

132. Dobin, A. et al. STAR: ultrafast universal RNA-seq aligner. Bioinformatics 29, 15-21 (2013).

133. Liao, Y., Smyth, G. K. \& Shi, W. Sequence analysis featureCounts : an efficient general purpose program for assigning sequence reads to genomic features. Bioinformatics 30, 923-930 (2014).

134. Love, M. I., Huber, W. \& Anders, S. Moderated estimation of fold change and dispersion for RNA-seq data with DESeq2. Genome Biol. 1-21 (2014). doi:10.1186/s13059-014-0550-8

135. Green, J., Pollard, J., Tugendreich, S. \& Kra, A. Systems biology Causal analysis approaches in Ingenuity Pathway Analysis. Bioinformatics 30, 523-530 (2014).

136. Asai, K. et al. Dexamethasone-induced suppression of aortic atherosclerosis in cholesterol-fed rabbits. Possible mechanisms. Atheroscler. Thromb. Vasc. Biol. 13, 892-899 (1993).

137. Chen, A. et al. Cutting Edge: Dexamethasone Potentiates the Responses of Both Regulatory T Cells and B-1 Cells to Antigen Immunization in the ApoE-/- Mouse Model of Atherosclerosis. J. Immunol. 193, 35-9 (2014).

138. Duval, C., Chinetti, G., Trottein, F., Fruchart, J. C. \& Staels, B. The role of PPARs in atherosclerosis. Trends Mol. Med. 8, 422-430 (2002).

139. Castrillo, A. \& Tontonoz, P. PPARs in atherosclerosis : the clot thickens. J. Clin. Invest. 114, 10-12 (2004).

140. Chawla, A. et al. A PPARy -LXR-ABCA1 pathway in macrophages is involved in cholesterol efflux and atherogenesis. Mol. Cell 7, 161-171 (2001).

141. Ohshima, K., Mogi, M. \& Horiuchi, M. Role of peroxisome proliferatoractivated receptor- $\mathrm{Y}$ in vascular inflammation. Int. J. Vasc. Med. 2012, 110 (2012). 
142. Li, A. C. et al. Peroxisome proliferator - activated receptor $y$ ligands inhibit development of atherosclerosis in LDL receptor - deficient mice. J. Clin. Invest. 106, 523-531 (2000).

143. Babaev, V. R. et al. Conditional Knockout of Macrophage PPAR y Increases Atherosclerosis in C57BL / 6 and Low-Density Lipoprotein Receptor - Deficient Mice. Arter. Thromb Vasc Biol 25, 1647-1653 (2005).

144. Allard C. van der Wal, A. E. B. Atherosclerotic plaque rupture - pathologic basis of plaque stability and instability. Cardiovasc Res 41, 334-344 (1999).

145. Skalén, K. et al. Subendothelial retention of atherogenic lipoproteins in early atherosclerosis. Nature 417, 750-4 (2002).

146. Borén, J. et al. Identification of the principal proteoglycan-binding site in LDL: A single-point mutation in apo-B100 severely affects proteoglycan interaction without affecting LDL receptor binding. J. Clin. Invest. 101, 2658-2664 (1998).

147. Sasaki, T. et al. Endostatins derived from collagens XV and XVIII differ in structural and binding properties, tissue distribution and anti-angiogenic activity. J. Mol. Biol. 301, 1179-1190 (2000).

148. Stoll, L. L., Denning, G. M. \& Weintraub, N. L. Potential role of endotoxin as a proinflammatory mediator of atherosclerosis. Arterioscler. Thromb. Vasc. Biol. 24, 2227-2236 (2004).

149. Boesten, L. et al. Tumor necrosis factor-alpha promotes atherosclerotic lesion progression in APOE ${ }^{\star} 3$-leiden transgenic mice. Cardiovasc. Res. 66, 179-185 (2005).

150. Robertson, A. L. et al. Disruption of TGF- $\beta$ signaling in T cells accelerates atherosclerosis. J. Clin. Invest. 112, 1342-1350 (2003).

151. Gupta, S. et al. IFN-y Potentiates Atherosclerosis in ApoE Knock-out Mice. J. Clin. Invest. 99, 2752-2761 (1997).

152. Alexander, M. R. et al. Genetic inactivation of IL-1 signaling enhances atherosclerotic plaque instability and reduces outward vessel remodeling in advanced atherosclerosis in mice. J. Clin. Invest. 122, 70-79 (2012).

153. Eklund, L. et al. Lack of type XV collagen causes a skeletal myopathy and cardiovascular defects in mice. Proc. Natl. Acad. Sci. 98, 1194-1199 (2001). 
154. Staudinger, L. A. et al. Interactions between the discoidin domain receptor 1 and b 1 integrin regulate attachment to collagen. (2013).

doi:10.1242/bio.20135090

155. Watkins, H. \& Farrall, M. Genetic susceptibility to coronary artery disease: from promise to progress. Nat. Rev. Genet. 7, 163-173 (2006).

156. Lepidi, S. et al. MMP9 production by human monocyte-derived macrophages is decreased on polymerized type I collagen. J. Vasc. Surg. 34, 1111-1118 (2001).

157. Wesley, R. B., Meng, X., Godin, D. \& Galis, Z. S. Extracellular matrix modulates macrophage functions characteristic to atheroma: collagen type I enhances acquisition of resident macrophage traits by human peripheral blood monocytes in vitro. Arterioscler. Thromb. Vasc. Biol. 18, 432-440 (1998).

158. McArdle, S., Chodaczek, G., Ray, N. \& Ley, K. Intravital live cell triggered imaging system reveals monocyte patrolling and macrophage migration in atherosclerotic arteries. J. Biomed. Opt. 20, 26005 (2015).

159. Amenta, P. S. et al. Loss of types XV and XIX collagen precedes basement membrane invasion in ductal carcinoma of the female breast. J. Pathol. 199, 298-308 (2003).

160. Hedrick, C. C. Lymphocytes in Atherosclerosis. Arterioscler. Thromb. Vasc. Biol. 35, 253-257 (2015).

161. Mallat, Z., Taleb, S., Ait-Oufella, H. \& Tedgui, A. The role of adaptive T cell immunity in atherosclerosis. J. Lipid Res. 50 Suppl, S364-S369 (2009).

162. Tsiantoulas, D., Diehl, C. J., Witztum, J. L. \& Binder, C. J. B cells and humoral immunity in atherosclerosis. Circ. Res. 114, 1743-1756 (2014).

163. Morris-Rosenfeld, S., Lipinski, M. J. \& McNamara, C. A. Understanding the role of B cells in atherosclerosis: potential clinical implications. Expert Rev. Clin. Immunol. 10, 77-89 (2014).

164. Weitkamp, B., Cullen, P., Plenz, G., Robenek, H. \& Rauterberg, J. Human macrophages synthesize type VIII collagen in vitro and in the atherosclerotic plaque. FASEB J. 13, 1445-57 (1999).

165. Schnoor, M. et al. Production of type VI collagen by human macrophages: a new dimension in macrophage functional heterogeneity. J. Immunol. 180, 5707-5719 (2008). 
166. Kusuma, S., Zhao, S. \& Gerecht, S. The extracellular matrix is a novel attribute of endothelial progenitors and of hypoxic mature endothelial cells. FASEB J. 26, 4925-4936 (2012).

167. Costa-Almeida, R. et al. Fibroblast-Endothelial Partners for Vascularization Strategies in Tissue Engineering. Tissue Eng. Part A 0, 1-11 (2014).

168. VanderLaan, P. A., Reardon, C. A. \& Getz, G. S. Site Specificity of Atherosclerosis: Site-Selective Responses to Atherosclerotic Modulators. Arterioscler. Thromb. Vasc. Biol. 24, 12-22 (2004).

169. Majesky, M. W. Developmental basis of vascular smooth muscle diversity. Arterioscler. Thromb. Vasc. Biol. 27, 1248-1258 (2007).

170. Baylis, R. A., Gomez, D. \& Owens, G. K. Shifting the Focus of Preclinical, Murine Atherosclerosis Studies From Prevention to Late-Stage Intervention. Circ. Res. 120, 775-777 (2017).

171. Vogel, W. F., Abdulhussein, R. \& Ford, C. E. Sensing extracellular matrix: An update on discoidin domain receptor function. Cell. Signal. 18, 11081116 (2006).

172. Harpaz, N., Ordan, E., Ocorr, K., Bodmer, R. \& Volk, T. Multiplexin Promotes Heart but Not Aorta Morphogenesis by Polarized Enhancement of Slit/Robo Activity at the Heart Lumen. PLoS Genet. 9, (2013).

173. Yuen, D. A. \& Robinson, L. A. Slit2-Robo signaling. Curr. Opin. Nephrol. Hypertens. 22, 445-451 (2013).

174. Hurskainen, M., Ruggiero, F., Hägg, P., Pihlajaniemi, T. \& Huhtala, P. Recombinant human collagen $\mathrm{XV}$ regulates cell adhesion and migration. $J$. Biol. Chem. 285, 5258-5265 (2010).

175. Skalén, K. et al. Subendothelial retention of atherogenic lipoproteins in early atherosclerosis. Nature 417, 750-4 (2002).

176. Flood, C. et al. Molecular Mechanism for Changes in Proteoglycan Binding on Compositional Changes of the Core and the Surface of Low-Density Lipoprotein-Containing Human Apolipoprotein B100. Arterioscler. Thromb. Vasc. Biol. 24, 564-570 (2004).

177. Flood, C. et al. Identification of the proteoglycan binding site in apolipoprotein B48. J. Biol. Chem. 277, 32228-32233 (2002). 
178. Gustafsson, M. et al. Retention of low-density lipoprotein in atherosclerotic lesions of the mouse: Evidence for a role of lipoprotein lipase. Circ. Res. 101, 777-783 (2007).

179. Ning, T. et al. Gene therapy with the angiogenesis inhibitor endostatin in an orthotopic lung cancer murine model. Hum. Gene Ther. 20, 103-111 (2009).

180. O'Reilly, M. S. et al. Endostatin: an endogenous inhibitor of angiogenesis and tumor growth. Cell 88, 277-285 (1997).

181. John, H., Preissner, K. T., Forssmann, W. G. \& Ständker, L. Novel glycosylated forms of human plasma endostatin and circulating endostatinrelated fragments of collagen XV. Biochemistry 38, 10217-10224 (1999).

182. Gutterman, D. D. et al. The Human Microcirculation: Regulation of Flow and beyond. Circ. Res. 118, 157-172 (2016).

183. Stamler, J., Neaton, J. D. \& Wentworth, D. N. Blood pressure (systolic and diastolic) and risk of fatal coronary heart disease. Hypertension 13, I2-12 (1989).

184. Chobanian, A. V. Effects of hypertension on arterial gene expression and atherosclerosis. Adv. Exp. Med. Biol. 308, 45-53 (1991).

185. Alexander, R. W. Hypertension and the Pathogenesis of Atherosclerosis. Hypertension 25, 155-161 (1995).

186. F.S., S. et al. Does the Relation of Blood Pressure to Coronary Heart Change With Aging? The Framingham Heart Study. Circulation 103, 12451249 (2001).

187. Kivirikko, S., Saarela, J., Myers, J. C., Autio-Harmainen, H. \& Pihlajaniemi, $\mathrm{T}$. Distribution of type XV collagen transcripts in human tissue and their production by muscle cells and fibroblasts. Am. J. Pathol. 147, 1500-1509 (1995).

188. Pasterkamp, G., den Ruijter, H. M. \& Libby, P. Temporal shifts in clinical presentation and underlying mechanisms of atherosclerotic disease. Nat. Rev. Cardiol. 14, 21-29 (2016).

189. Lafont, A. Basic aspects of plaque vulnerability. Heart 89, 1262-7 (2003). 
190. van der Wal, a C., Becker, a E., van der Loos, C. M. \& Das, P. K. Site of intimal rupture or erosion of thrombosed coronary atherosclerotic plaques is characterized by an inflammatory process irrespective of the dominant plaque morphology. Circulation 89, 36-44 (1994). 